\title{
Imunology data
}

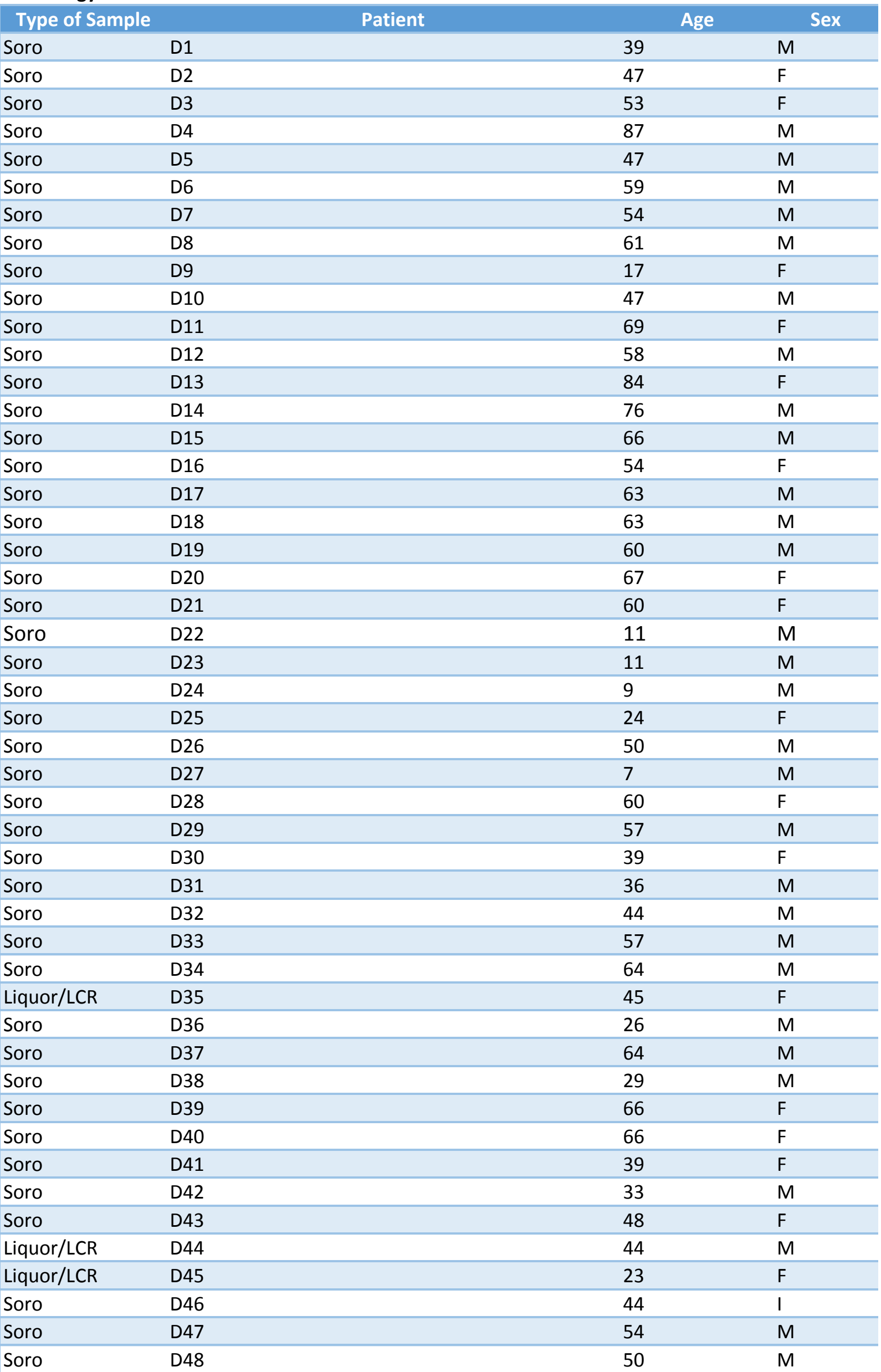




\begin{tabular}{|c|c|c|c|}
\hline Soro & D49 & 59 & $\mathrm{~F}$ \\
\hline Liquor/LCR & D50 & 82 & $\mathrm{~F}$ \\
\hline Soro & D51 & 50 & $\mathrm{~F}$ \\
\hline Soro & D52 & 39 & $\mathrm{~F}$ \\
\hline Soro & D53 & 36 & $M$ \\
\hline Soro & D54 & 11 & $M$ \\
\hline Soro & D55 & 35 & $\mathrm{~F}$ \\
\hline Soro & D56 & 42 & $M$ \\
\hline Soro & D57 & 42 & $\mathrm{~F}$ \\
\hline Soro & D58 & 56 & $M$ \\
\hline Soro & D59 & 69 & $M$ \\
\hline Soro & D60 & 19 & $M$ \\
\hline Soro & D61 & 56 & $\mathrm{~F}$ \\
\hline Soro & D62 & 4 & $M$ \\
\hline Soro & D63 & 13 & $M$ \\
\hline Soro & D64 & 48 & $M$ \\
\hline Soro & D65 & 75 & $M$ \\
\hline Soro & D66 & 45 & $M$ \\
\hline Soro & D67 & 61 & $\mathrm{~F}$ \\
\hline Soro & D68 & 39 & $M$ \\
\hline Soro & D69 & 34 & M \\
\hline Soro & D70 & 76 & $M$ \\
\hline Soro & D71 & 76 & M \\
\hline Soro & D72 & 44 & M \\
\hline Soro & D73 & 65 & M \\
\hline Soro & D74 & 11 & M \\
\hline Soro & D75 & 0 & $\mathrm{~F}$ \\
\hline Soro & D76 & 64 & M \\
\hline Soro & D77 & 72 & $\mathrm{~F}$ \\
\hline Soro & D78 & 74 & $M$ \\
\hline Soro & D79 & 67 & $M$ \\
\hline Soro & $\mathrm{D} 80$ & 71 & $\mathrm{~F}$ \\
\hline Soro & D81 & 68 & $\mathrm{~F}$ \\
\hline Soro & D82 & 63 & $M$ \\
\hline Soro & D83 & 42 & $\mathrm{~F}$ \\
\hline Soro & D84 & 84 & $\mathrm{~F}$ \\
\hline Soro & D85 & 85 & M \\
\hline Soro & D86 & 52 & $M$ \\
\hline Soro & D87 & 68 & $M$ \\
\hline Soro & D88 & 67 & $\mathrm{~F}$ \\
\hline Soro & D89 & 67 & $\mathrm{~F}$ \\
\hline Soro & D90 & 48 & $M$ \\
\hline Soro & D91 & 74 & $M$ \\
\hline Soro & D92 & 16 & $M$ \\
\hline Soro & D93 & 32 & $M$ \\
\hline Soro & D94 & 61 & $\mathrm{~F}$ \\
\hline Soro & D95 & 60 & $\mathrm{~F}$ \\
\hline Soro & D96 & 68 & $\mathrm{~F}$ \\
\hline Soro & D97 & 56 & $M$ \\
\hline Soro & D98 & 42 & $M$ \\
\hline
\end{tabular}




\begin{tabular}{|c|c|c|c|}
\hline Soro & D99 & 45 & $M$ \\
\hline Soro & D100 & 76 & $M$ \\
\hline Soro & D101 & 21 & M \\
\hline Soro & D102 & 74 & M \\
\hline Soro & D103 & 73 & M \\
\hline Soro & D104 & 60 & $M$ \\
\hline Soro & D105 & 52 & $\mathrm{~F}$ \\
\hline Soro & D106 & 65 & M \\
\hline Soro & D107 & 65 & M \\
\hline Soro & D108 & 58 & $M$ \\
\hline Soro & D109 & 64 & M \\
\hline Soro & D110 & 18 & $\mathrm{~F}$ \\
\hline Soro & D111 & 72 & M \\
\hline Liquor/LCR & D112 & 58 & $\mathrm{~F}$ \\
\hline Soro & D113 & 79 & $\mathrm{~F}$ \\
\hline Soro & D114 & 82 & $\mathrm{~F}$ \\
\hline Soro & D115 & 18 & $\mathrm{~F}$ \\
\hline Liquor/LCR & D116 & 60 & $\mathrm{~F}$ \\
\hline Soro & D117 & 78 & $\mathrm{~F}$ \\
\hline Liquor/LCR & D118 & 72 & M \\
\hline Soro & D119 & 62 & M \\
\hline Soro & D120 & 75 & $\mathrm{M}$ \\
\hline Soro & D121 & 11 & M \\
\hline Soro & D122 & 76 & M \\
\hline Soro & D123 & 48 & M \\
\hline Soro & D124 & 59 & $\mathrm{~F}$ \\
\hline Soro & D125 & 17 & $\mathrm{~F}$ \\
\hline Soro & D126 & 53 & M \\
\hline Soro & D127 & 53 & M \\
\hline Soro & D128 & 7 & 1 \\
\hline Soro & D129 & 60 & M \\
\hline Soro & D130 & 73 & $\mathrm{~F}$ \\
\hline Soro & D131 & 40 & M \\
\hline Soro & D132 & 83 & M \\
\hline Liquor/LCR & D133 & 66 & M \\
\hline Soro & D134 & 86 & $\mathrm{~F}$ \\
\hline Soro & D135 & 83 & $\mathrm{~F}$ \\
\hline Soro & D136 & 65 & $\mathrm{~F}$ \\
\hline Soro & D137 & 84 & $\mathrm{~F}$ \\
\hline Soro & D138 & 21 & M \\
\hline Soro & D139 & 46 & $\mathrm{~F}$ \\
\hline Soro & D140 & 77 & M \\
\hline Soro & D141 & 30 & M \\
\hline Soro & D142 & 75 & M \\
\hline Soro & D143 & 56 & M \\
\hline Soro & D144 & 41 & $\mathrm{~F}$ \\
\hline Soro & D145 & 25 & M \\
\hline Soro & D146 & 15 & M \\
\hline Soro & D147 & 28 & M \\
\hline Soro & D148 & 38 & $\mathrm{~F}$ \\
\hline
\end{tabular}




\begin{tabular}{|c|c|c|c|}
\hline Soro & D149 & 69 & $\mathrm{~F}$ \\
\hline Soro & D150 & 84 & $\mathrm{~F}$ \\
\hline Liquor/LCR & D151 & 31 & M \\
\hline Liquor/LCR & D152 & 31 & $M$ \\
\hline Soro & D153 & 75 & M \\
\hline Soro & D154 & 55 & $\mathrm{~F}$ \\
\hline Soro & D155 & 84 & $M$ \\
\hline Soro & D156 & 57 & M \\
\hline Soro & D157 & 22 & M \\
\hline Soro & D158 & 50 & $\mathrm{~F}$ \\
\hline Soro & D159 & 60 & M \\
\hline Liquor/LCR & D160 & 80 & $\mathrm{~F}$ \\
\hline Soro & D161 & 32 & $\mathrm{~F}$ \\
\hline Liquor/LCR & D162 & 42 & $M$ \\
\hline Liquor/LCR & D163 & 42 & M \\
\hline Liquor/LCR & D164 & 81 & $\mathrm{~F}$ \\
\hline Soro & D165 & 80 & M \\
\hline Soro & D166 & 65 & $M$ \\
\hline Soro & D167 & 40 & $\mathrm{~F}$ \\
\hline Soro & D168 & 75 & $\mathrm{~F}$ \\
\hline Soro & D169 & 23 & $\mathrm{~F}$ \\
\hline Soro & D170 & 59 & $\mathrm{~F}$ \\
\hline Soro & D171 & 34 & $\mathrm{~F}$ \\
\hline Soro & D172 & 66 & $\mathrm{~F}$ \\
\hline Soro & D173 & 64 & M \\
\hline Soro & D174 & 78 & M \\
\hline Soro & D175 & 40 & $\mathrm{~F}$ \\
\hline Soro & D176 & 24 & M \\
\hline Soro & D177 & 17 & $\mathrm{~F}$ \\
\hline Soro & D178 & 40 & $\mathrm{~F}$ \\
\hline Liquor/LCR & D179 & 59 & M \\
\hline Soro & D180 & 38 & $\mathrm{~F}$ \\
\hline Soro & D181 & 54 & M \\
\hline Soro & D182 & 44 & M \\
\hline Soro & D183 & 44 & M \\
\hline Soro & D184 & 31 & $\mathrm{~F}$ \\
\hline Soro & D185 & 58 & $\mathrm{~F}$ \\
\hline Soro & D186 & 62 & M \\
\hline Soro & D187 & 46 & $\mathrm{~F}$ \\
\hline Soro & D188 & 53 & M \\
\hline Soro & D189 & 91 & $M$ \\
\hline Soro & D190 & 71 & M \\
\hline Liquor/LCR & D191 & 0 & $\mathrm{~F}$ \\
\hline Soro & D192 & 46 & $\mathrm{~F}$ \\
\hline Soro & D193 & 24 & $\mathrm{~F}$ \\
\hline Soro & D194 & 29 & $M$ \\
\hline Soro & D195 & 42 & M \\
\hline Soro & D196 & 41 & M \\
\hline Soro & D197 & 40 & $\mathrm{~F}$ \\
\hline Soro & D198 & 57 & $M$ \\
\hline
\end{tabular}




\begin{tabular}{|c|c|c|c|}
\hline Soro & D199 & 41 & M \\
\hline Soro & D200 & 40 & $\mathrm{~F}$ \\
\hline Soro & D201 & 63 & M \\
\hline Soro & D202 & 46 & M \\
\hline Soro & D203 & 46 & M \\
\hline Soro & D204 & 36 & $M$ \\
\hline Liquor/LCR & D205 & 47 & $\mathrm{~F}$ \\
\hline Soro & D206 & 38 & $\mathrm{~F}$ \\
\hline Soro & D207 & 38 & $\mathrm{~F}$ \\
\hline Liquor/LCR & D208 & 68 & M \\
\hline Soro & D209 & 60 & $\mathrm{~F}$ \\
\hline Soro & D210 & 55 & $\mathrm{~F}$ \\
\hline Soro & D211 & 55 & $\mathrm{~F}$ \\
\hline Soro & D212 & 43 & $\mathrm{M}$ \\
\hline Soro & D213 & 82 & M \\
\hline Soro & D214 & 20 & M \\
\hline Soro & D215 & 52 & $\mathrm{~F}$ \\
\hline Soro & D216 & 19 & M \\
\hline Soro & D217 & 20 & $\mathrm{~F}$ \\
\hline Soro & D218 & 35 & M \\
\hline Soro & D219 & 77 & M \\
\hline Soro & D220 & 64 & M \\
\hline Liquor/LCR & D221 & 60 & M \\
\hline Soro & D222 & 9 & $\mathrm{~F}$ \\
\hline Liquor/LCR & D223 & 39 & $\mathrm{~F}$ \\
\hline Soro & D224 & 70 & M \\
\hline Soro & D225 & 43 & $\mathrm{~F}$ \\
\hline Soro & D226 & 43 & M \\
\hline Soro & D227 & 67 & M \\
\hline Soro & D228 & 73 & $\mathrm{M}$ \\
\hline Soro & D229 & 48 & $\mathrm{~F}$ \\
\hline Soro & D230 & 47 & $\mathrm{~F}$ \\
\hline Soro & D231 & 40 & M \\
\hline Soro & D232 & 65 & $\mathrm{~F}$ \\
\hline Soro & D233 & 68 & $\mathrm{~F}$ \\
\hline Soro & D234 & 78 & $M$ \\
\hline Liquor/LCR & D235 & 66 & M \\
\hline Soro & D236 & 41 & M \\
\hline Soro & D237 & 76 & $M$ \\
\hline Soro & D238 & 69 & M \\
\hline Soro & D239 & 61 & M \\
\hline Soro & D240 & 36 & M \\
\hline Soro & D241 & 15 & $\mathrm{~F}$ \\
\hline Soro & D242 & 67 & $\mathrm{~F}$ \\
\hline Soro & D243 & 64 & M \\
\hline Soro & D244 & 69 & $\mathrm{M}$ \\
\hline Soro & D245 & 20 & $M$ \\
\hline Soro & D246 & 85 & $M$ \\
\hline Soro & D247 & 51 & M \\
\hline Soro & D248 & 58 & M \\
\hline
\end{tabular}




\begin{tabular}{|c|c|c|c|}
\hline Soro & D249 & 26 & $M$ \\
\hline Soro & D250 & 70 & $M$ \\
\hline Soro & D251 & 70 & $M$ \\
\hline Soro & D252 & 79 & $\mathrm{~F}$ \\
\hline Soro & D253 & 69 & M \\
\hline Soro & D254 & 73 & M \\
\hline Soro & D255 & 78 & $\mathrm{~F}$ \\
\hline Soro & D256 & 49 & $\mathrm{~F}$ \\
\hline Soro & D257 & 46 & $\mathrm{~F}$ \\
\hline Soro & D258 & 41 & $\mathrm{~F}$ \\
\hline Soro & D259 & 19 & M \\
\hline Soro & D260 & 68 & $M$ \\
\hline Soro & D261 & 63 & $\mathrm{~F}$ \\
\hline Soro & D262 & 73 & $\mathrm{~F}$ \\
\hline Soro & D263 & 52 & $\mathrm{~F}$ \\
\hline Soro & D264 & 36 & $\mathrm{~F}$ \\
\hline Soro & D265 & 65 & $M$ \\
\hline Soro & D266 & 46 & $M$ \\
\hline Soro & D267 & 84 & $\mathrm{~F}$ \\
\hline Soro & D268 & 74 & $\mathrm{~F}$ \\
\hline Liquor/LCR & D269 & 58 & M \\
\hline Soro & D270 & 56 & $\mathrm{~F}$ \\
\hline Soro & D271 & 64 & $M$ \\
\hline Soro & D272 & 34 & $\mathrm{~F}$ \\
\hline Soro & D273 & 70 & $\mathrm{~F}$ \\
\hline Soro & D274 & 45 & $M$ \\
\hline Soro & D275 & 55 & $\mathrm{~F}$ \\
\hline Soro & D276 & 66 & M \\
\hline Soro & D277 & 48 & $\mathrm{~F}$ \\
\hline Liquor/LCR & D278 & 22 & $\mathrm{M}$ \\
\hline Soro & D279 & 63 & $M$ \\
\hline Soro & D280 & 76 & $\mathrm{~F}$ \\
\hline Soro & D281 & 95 & $M$ \\
\hline Soro & D282 & 59 & $\mathrm{~F}$ \\
\hline Soro & D283 & 30 & $\mathrm{~F}$ \\
\hline Soro & D284 & 57 & M \\
\hline Soro & D285 & 74 & $\mathrm{~F}$ \\
\hline Soro & D286 & 74 & $\mathrm{~F}$ \\
\hline Soro & D287 & 58 & $\mathrm{~F}$ \\
\hline Soro & D288 & 43 & $\mathrm{~F}$ \\
\hline Soro & D289 & 59 & $M$ \\
\hline Soro & D290 & 67 & $\mathrm{~F}$ \\
\hline Soro & D291 & 63 & $\mathrm{~F}$ \\
\hline Soro & D292 & 7 & M \\
\hline Soro & D293 & 56 & $\mathrm{~F}$ \\
\hline Soro & D294 & 42 & $M$ \\
\hline Soro & D295 & 41 & $M$ \\
\hline Soro & D296 & 0 & $M$ \\
\hline Soro & D297 & 54 & M \\
\hline Soro & D298 & 47 & $\mathrm{~F}$ \\
\hline
\end{tabular}




\begin{tabular}{|c|c|c|c|}
\hline Soro & D299 & 49 & $\mathrm{~F}$ \\
\hline Soro & D300 & 30 & $\mathrm{~F}$ \\
\hline Soro & D301 & 53 & $\mathrm{~F}$ \\
\hline Soro & D302 & 68 & M \\
\hline Soro & D303 & 41 & M \\
\hline Soro & D304 & 44 & $\mathrm{~F}$ \\
\hline Soro & D305 & 85 & M \\
\hline Liquor/LCR & D306 & 79 & M \\
\hline Soro & D307 & 63 & $\mathrm{~F}$ \\
\hline Soro & D308 & 0 & $M$ \\
\hline Soro & D309 & 33 & $\mathrm{~F}$ \\
\hline Soro & D310 & 80 & M \\
\hline Soro & D311 & 43 & M \\
\hline Soro & D312 & 37 & $\mathrm{~F}$ \\
\hline Soro & D313 & 46 & M \\
\hline Soro & D314 & 23 & $\mathrm{~F}$ \\
\hline Soro & D315 & 65 & $\mathrm{~F}$ \\
\hline Soro & D316 & 79 & $\mathrm{~F}$ \\
\hline Soro & D317 & 52 & $\mathrm{~F}$ \\
\hline Soro & D318 & 54 & M \\
\hline Liquor/LCR & D319 & 74 & M \\
\hline Soro & D320 & 37 & $\mathrm{~F}$ \\
\hline Soro & D321 & 74 & $\mathrm{~F}$ \\
\hline Soro & D322 & 67 & $\mathrm{~F}$ \\
\hline Liquor/LCR & D323 & 0 & $\mathrm{~F}$ \\
\hline Soro & D324 & 56 & M \\
\hline Soro & D325 & 41 & $\mathrm{~F}$ \\
\hline Soro & D326 & 71 & $\mathrm{~F}$ \\
\hline Soro & D327 & 58 & $\mathrm{~F}$ \\
\hline Soro & D328 & 51 & $\mathrm{~F}$ \\
\hline Soro & D329 & 59 & $\mathrm{~F}$ \\
\hline Soro & D330 & 15 & $M$ \\
\hline Soro & D331 & 69 & M \\
\hline Soro & D332 & 81 & M \\
\hline Soro & D333 & 49 & M \\
\hline Soro & D334 & 41 & M \\
\hline Soro & D335 & 33 & M \\
\hline Soro & D336 & 60 & M \\
\hline Soro & D337 & 14 & $M$ \\
\hline Soro & D338 & 26 & M \\
\hline Soro & D339 & 45 & M \\
\hline Soro & D340 & 51 & $\mathrm{~F}$ \\
\hline Soro & D341 & 21 & M \\
\hline Soro & D342 & 27 & $\mathrm{~F}$ \\
\hline Soro & D343 & 50 & M \\
\hline Soro & D344 & 38 & $\mathrm{~F}$ \\
\hline Soro & D345 & 79 & $\mathrm{~F}$ \\
\hline Soro & D346 & 61 & M \\
\hline Soro & D347 & 78 & $\mathrm{~F}$ \\
\hline Soro & D348 & 38 & $\mathrm{~F}$ \\
\hline
\end{tabular}




\begin{tabular}{|c|c|c|c|}
\hline Soro & D349 & 77 & $\mathrm{~F}$ \\
\hline Soro & D350 & 38 & $\mathrm{~F}$ \\
\hline Soro & D351 & 45 & $\mathrm{~F}$ \\
\hline Soro & D352 & 78 & $\mathrm{~F}$ \\
\hline Liquor/LCR & D353 & 67 & M \\
\hline Liquor/LCR & D354 & 29 & $\mathrm{~F}$ \\
\hline Soro & D355 & 31 & M \\
\hline Soro & D356 & 57 & M \\
\hline Soro & D357 & 84 & $\mathrm{~F}$ \\
\hline Soro & D358 & 34 & M \\
\hline Soro & D359 & 48 & $\mathrm{~F}$ \\
\hline Soro & D360 & 83 & M \\
\hline Soro & D361 & 70 & $\mathrm{~F}$ \\
\hline Liquor/LCR & D362 & 47 & M \\
\hline Liquor/LCR & D363 & 54 & M \\
\hline Soro & D364 & 85 & $\mathrm{~F}$ \\
\hline Soro & D365 & 60 & $\mathrm{~F}$ \\
\hline Soro & D366 & 52 & M \\
\hline Soro & D367 & 78 & $\mathrm{~F}$ \\
\hline Soro & D368 & 46 & M \\
\hline Soro & D369 & 61 & M \\
\hline Soro & D370 & 50 & M \\
\hline Soro & D371 & 12 & M \\
\hline Soro & D372 & 38 & $\mathrm{~F}$ \\
\hline Soro & D373 & 60 & $\mathrm{~F}$ \\
\hline Soro & D374 & 67 & M \\
\hline Soro & D375 & 60 & M \\
\hline Soro & D376 & 55 & $\mathrm{~F}$ \\
\hline Soro & D377 & 66 & $\mathrm{~F}$ \\
\hline Soro & D378 & 29 & M \\
\hline Soro & D379 & 66 & M \\
\hline Soro & D380 & 49 & $\mathrm{~F}$ \\
\hline Soro & D381 & 39 & $\mathrm{~F}$ \\
\hline Soro & D382 & 28 & $\mathrm{~F}$ \\
\hline Soro & D383 & 26 & M \\
\hline Soro & D384 & 67 & $\mathrm{~F}$ \\
\hline Soro & D385 & 81 & M \\
\hline Soro & D386 & 69 & M \\
\hline Soro & D387 & 50 & $\mathrm{~F}$ \\
\hline Soro & D388 & 58 & M \\
\hline Soro & D389 & 48 & M \\
\hline Soro & D390 & 53 & $\mathrm{~F}$ \\
\hline Soro & D391 & 85 & $\mathrm{~F}$ \\
\hline Soro & D392 & 46 & $\mathrm{~F}$ \\
\hline Soro & D393 & 49 & $\mathrm{~F}$ \\
\hline Soro & D394 & 28 & M \\
\hline Soro & D395 & 56 & $\mathrm{~F}$ \\
\hline Soro & D396 & 59 & $\mathrm{~F}$ \\
\hline Soro & D397 & 26 & $\mathrm{~F}$ \\
\hline Liquor/LCR & D398 & 33 & M \\
\hline
\end{tabular}




\begin{tabular}{|c|c|c|c|}
\hline Soro & D399 & 18 & $M$ \\
\hline Soro & D400 & 38 & $\mathrm{~F}$ \\
\hline Soro & D401 & 10 & $\mathrm{~F}$ \\
\hline Soro & D402 & 12 & $\mathrm{~F}$ \\
\hline Soro & D403 & 54 & M \\
\hline Soro & D404 & 72 & $M$ \\
\hline Soro & D405 & 77 & M \\
\hline Soro & D406 & 53 & M \\
\hline Liquor/LCR & D407 & 79 & M \\
\hline Soro & D408 & 44 & $\mathrm{~F}$ \\
\hline Soro & D409 & 57 & M \\
\hline Soro & D410 & 25 & $\mathrm{~F}$ \\
\hline Soro & D411 & 55 & $\mathrm{~F}$ \\
\hline Soro & D412 & 43 & $\mathrm{~F}$ \\
\hline Soro & D413 & 34 & $\mathrm{~F}$ \\
\hline Soro & D414 & 54 & $M$ \\
\hline Soro & D415 & 61 & $\mathrm{~F}$ \\
\hline Soro & D416 & 83 & $\mathrm{~F}$ \\
\hline Soro & D417 & 63 & $\mathrm{~F}$ \\
\hline Liquor/LCR & D418 & 0 & M \\
\hline Soro & D419 & 54 & M \\
\hline Soro & D420 & 27 & $\mathrm{~F}$ \\
\hline Soro & D421 & 26 & $\mathrm{~F}$ \\
\hline Soro & D422 & 26 & $\mathrm{~F}$ \\
\hline Soro & D423 & 42 & $\mathrm{~F}$ \\
\hline Soro & D424 & 51 & $\mathrm{~F}$ \\
\hline Soro & D425 & 71 & M \\
\hline Soro & D426 & 58 & M \\
\hline Liquor/LCR & D427 & 41 & M \\
\hline Soro & D428 & 32 & M \\
\hline Soro & D429 & 49 & M \\
\hline Soro & D430 & 61 & $M$ \\
\hline Soro & D431 & 61 & M \\
\hline Soro & D432 & 61 & $\mathrm{~F}$ \\
\hline Soro & D433 & 56 & $\mathrm{~F}$ \\
\hline Soro & D434 & 47 & $M$ \\
\hline Soro & D435 & 83 & $\mathrm{~F}$ \\
\hline Soro & D436 & 3 & $\mathrm{~F}$ \\
\hline Liquor/LCR & D437 & 81 & $M$ \\
\hline Soro & D438 & 40 & M \\
\hline Liquor/LCR & D439 & 47 & $\mathrm{~F}$ \\
\hline Soro & D440 & 38 & 1 \\
\hline Soro & D441 & 61 & M \\
\hline Soro & D442 & 44 & $\mathrm{~F}$ \\
\hline Soro & D443 & 79 & $\mathrm{~F}$ \\
\hline Soro & D444 & 56 & M \\
\hline Soro & D445 & 35 & M \\
\hline Soro & D446 & 58 & M \\
\hline Soro & D447 & 42 & $\mathrm{~F}$ \\
\hline Soro & D448 & 63 & $\mathrm{~F}$ \\
\hline
\end{tabular}




\begin{tabular}{|c|c|c|c|}
\hline Soro & D449 & 40 & $M$ \\
\hline Soro & D450 & 73 & $\mathrm{~F}$ \\
\hline Soro & D451 & 48 & M \\
\hline Soro & D452 & 14 & M \\
\hline Soro & D453 & 55 & M \\
\hline Soro & D454 & 3 & $\mathrm{~F}$ \\
\hline Liquor/LCR & D455 & 49 & M \\
\hline Soro & D456 & 13 & M \\
\hline Soro & D457 & 13 & M \\
\hline Soro & D458 & 54 & $M$ \\
\hline Soro & D459 & 50 & $\mathrm{~F}$ \\
\hline Soro & D460 & 60 & M \\
\hline Soro & D461 & 55 & M \\
\hline Soro & D462 & 64 & $\mathrm{~F}$ \\
\hline Liquor/LCR & D463 & 65 & $\mathrm{~F}$ \\
\hline Soro & D464 & 42 & M \\
\hline Soro & D465 & 62 & $\mathrm{~F}$ \\
\hline Soro & D466 & 25 & M \\
\hline Soro & D467 & 65 & M \\
\hline Soro & D468 & 41 & M \\
\hline Soro & D469 & 12 & M \\
\hline Soro & D470 & 54 & M \\
\hline Soro & D471 & 61 & M \\
\hline Soro & D472 & 68 & $\mathrm{~F}$ \\
\hline Soro & D473 & 41 & M \\
\hline Soro & D474 & 79 & M \\
\hline Soro & D475 & 48 & M \\
\hline Soro & D476 & 56 & M \\
\hline Liquor/LCR & D477 & 65 & M \\
\hline Soro & D478 & 79 & $\mathrm{~F}$ \\
\hline Soro & D479 & 58 & M \\
\hline Soro & D480 & 33 & $M$ \\
\hline Soro & D481 & 34 & M \\
\hline Soro & D482 & 31 & $\mathrm{~F}$ \\
\hline Soro & D483 & 85 & M \\
\hline Liquor/LCR & D484 & 79 & $M$ \\
\hline Soro & D485 & 15 & $\mathrm{~F}$ \\
\hline Soro & D486 & 38 & M \\
\hline Soro & D487 & 65 & $M$ \\
\hline Soro & D488 & 69 & M \\
\hline Soro & D489 & 78 & M \\
\hline Soro & D490 & 69 & $\mathrm{~F}$ \\
\hline Soro & D491 & 16 & M \\
\hline Soro & D492 & 76 & $\mathrm{~F}$ \\
\hline Soro & D493 & 83 & M \\
\hline Soro & D494 & 68 & $M$ \\
\hline Soro & D495 & 70 & M \\
\hline Soro & D496 & 32 & $\mathrm{~F}$ \\
\hline Soro & D497 & 38 & $\mathrm{~F}$ \\
\hline Soro & D498 & 28 & M \\
\hline
\end{tabular}




\begin{tabular}{|c|c|c|c|}
\hline Soro & D499 & 24 & $M$ \\
\hline Liquor/LCR & D500 & 47 & $M$ \\
\hline Soro & D501 & 62 & $\mathrm{~F}$ \\
\hline Liquor/LCR & D502 & 71 & $\mathrm{~F}$ \\
\hline Soro & D503 & 34 & M \\
\hline Soro & D504 & 34 & $M$ \\
\hline Soro & D505 & 50 & $\mathrm{~F}$ \\
\hline Soro & D506 & 34 & $\mathrm{~F}$ \\
\hline Liquor/LCR & D507 & 88 & $\mathrm{~F}$ \\
\hline Soro & D508 & 44 & $M$ \\
\hline Soro & D509 & 44 & $\mathrm{~F}$ \\
\hline Soro & D510 & 42 & M \\
\hline Soro & D511 & 38 & M \\
\hline Liquor/LCR & D512 & 40 & M \\
\hline Soro & D513 & 70 & $\mathrm{~F}$ \\
\hline Liquor/LCR & D514 & 61 & M \\
\hline Soro & D515 & 50 & $\mathrm{~F}$ \\
\hline Soro & D516 & 75 & M \\
\hline Soro & D517 & 79 & M \\
\hline Soro & D518 & 57 & M \\
\hline Soro & D519 & 61 & M \\
\hline Soro & D520 & 47 & $\mathrm{~F}$ \\
\hline Soro & D521 & 39 & $\mathrm{~F}$ \\
\hline Soro & D522 & 38 & $\mathrm{~F}$ \\
\hline Soro & D523 & 75 & $\mathrm{~F}$ \\
\hline Liquor/LCR & D524 & 61 & M \\
\hline Liquor/LCR & D525 & 42 & M \\
\hline Soro & D526 & 73 & M \\
\hline Soro & D527 & 61 & M \\
\hline Liquor/LCR & D528 & 57 & M \\
\hline Soro & D529 & 90 & $\mathrm{~F}$ \\
\hline Liquor/LCR & D530 & 0 & $M$ \\
\hline Soro & D531 & 73 & $\mathrm{~F}$ \\
\hline Soro & D532 & 66 & M \\
\hline Soro & D533 & 69 & M \\
\hline Soro & D534 & 63 & M \\
\hline Soro & D535 & 67 & $\mathrm{~F}$ \\
\hline Soro & D536 & 40 & $\mathrm{~F}$ \\
\hline Soro & D537 & 76 & $\mathrm{~F}$ \\
\hline Soro & D538 & 76 & $\mathrm{~F}$ \\
\hline Soro & D539 & 61 & M \\
\hline Soro & D540 & 65 & M \\
\hline Soro & D541 & 63 & $\mathrm{~F}$ \\
\hline Soro & D542 & 40 & $\mathrm{~F}$ \\
\hline Soro & D543 & 76 & $\mathrm{~F}$ \\
\hline Soro & D544 & 42 & M \\
\hline Soro & D545 & 60 & $M$ \\
\hline Soro & D546 & 56 & $M$ \\
\hline Soro & D547 & 56 & $\mathrm{~F}$ \\
\hline Soro & D548 & 80 & $\mathrm{~F}$ \\
\hline
\end{tabular}




\begin{tabular}{|c|c|c|c|}
\hline Soro & D549 & 75 & $\mathrm{~F}$ \\
\hline Liquor/LCR & D550 & 82 & $\mathrm{~F}$ \\
\hline Soro & D551 & 72 & $M$ \\
\hline Soro & D552 & 27 & M \\
\hline Liquor/LCR & D553 & 46 & $\mathrm{~F}$ \\
\hline Soro & D554 & 83 & $M$ \\
\hline Soro & D555 & 52 & $M$ \\
\hline Soro & D556 & 68 & M \\
\hline Soro & D557 & 77 & $\mathrm{~F}$ \\
\hline Liquor/LCR & D558 & 54 & $\mathrm{~F}$ \\
\hline Soro & D559 & 56 & M \\
\hline Soro & D560 & 63 & $\mathrm{~F}$ \\
\hline Soro & D561 & 81 & $\mathrm{~F}$ \\
\hline Soro & D562 & 66 & $M$ \\
\hline Soro & D563 & 37 & M \\
\hline Soro & D564 & 50 & $M$ \\
\hline Soro & D565 & 54 & $M$ \\
\hline Soro & D566 & 39 & M \\
\hline Soro & D567 & 66 & M \\
\hline Soro & D568 & 71 & $\mathrm{~F}$ \\
\hline Soro & D569 & 45 & 1 \\
\hline Soro & D570 & 53 & M \\
\hline Soro & D571 & 21 & $\mathrm{~F}$ \\
\hline Soro & D572 & 33 & $\mathrm{~F}$ \\
\hline Soro & D573 & 58 & $\mathrm{~F}$ \\
\hline Soro & D574 & 43 & $\mathrm{~F}$ \\
\hline Soro & D575 & 43 & $\mathrm{~F}$ \\
\hline Soro & D576 & 44 & $\mathrm{~F}$ \\
\hline Soro & D577 & 22 & M \\
\hline Soro & D578 & 76 & $\mathrm{~F}$ \\
\hline Soro & D579 & 57 & $\mathrm{~F}$ \\
\hline Soro & D580 & 63 & $\mathrm{~F}$ \\
\hline Soro & D581 & 65 & $M$ \\
\hline Soro & D582 & 43 & $M$ \\
\hline Soro & D583 & 46 & $\mathrm{~F}$ \\
\hline Soro & D584 & 58 & $M$ \\
\hline Soro & D585 & 48 & M \\
\hline Soro & D586 & 65 & $M$ \\
\hline Soro & D587 & 63 & $\mathrm{~F}$ \\
\hline Liquor/LCR & D588 & 34 & $M$ \\
\hline Soro & D589 & 51 & M \\
\hline Soro & D590 & 38 & $\mathrm{~F}$ \\
\hline Soro & D591 & 5 & $M$ \\
\hline Soro & D592 & 61 & $\mathrm{~F}$ \\
\hline Soro & D593 & 45 & $\mathrm{~F}$ \\
\hline Liquor/LCR & D594 & 59 & $\mathrm{~F}$ \\
\hline Soro & D595 & 42 & $M$ \\
\hline Soro & D596 & 55 & M \\
\hline Soro & D597 & 82 & M \\
\hline Soro & D598 & 84 & $\mathrm{~F}$ \\
\hline
\end{tabular}




\begin{tabular}{|c|c|c|c|}
\hline Soro & D599 & 61 & $M$ \\
\hline Soro & D600 & 8 & $F$ \\
\hline Soro & D601 & 85 & $F$ \\
\hline Soro & D602 & 73 & $F$ \\
\hline Soro & D603 & 78 & $\mathrm{~F}$ \\
\hline Soro & D604 & 81 & $M$ \\
\hline Soro & D605 & 40 & $F$ \\
\hline Soro & D606 & 70 & $M$ \\
\hline Soro & D607 & 57 & $M$ \\
\hline Soro & D608 & 57 & $M$ \\
\hline Soro & D609 & 49 & $F$ \\
\hline Soro & D610 & 42 & $\mathrm{M}$ \\
\hline Soro & D611 & 36 & $\mathrm{M}$ \\
\hline Soro & D612 & 24 & $\mathrm{~F}$ \\
\hline Soro & D613 & 80 & $F$ \\
\hline Soro & D614 & 61 & $M$ \\
\hline Soro & D615 & 62 & $F$ \\
\hline Soro & D616 & 79 & $\mathrm{M}$ \\
\hline Soro & D617 & 26 & $F$ \\
\hline Soro & D618 & 84 & $\mathrm{M}$ \\
\hline Soro & D619 & 59 & $\mathrm{~F}$ \\
\hline Soro & D620 & 24 & $F$ \\
\hline Soro & D621 & 62 & $F$ \\
\hline Soro & D622 & 49 & $\mathrm{~F}$ \\
\hline Soro & D623 & 8 & $\mathrm{M}$ \\
\hline Soro & D624 & 42 & $F$ \\
\hline Soro & D625 & 41 & $F$ \\
\hline Soro & D626 & 36 & $\mathrm{M}$ \\
\hline Soro & D627 & 68 & $M$ \\
\hline Soro & D628 & 46 & $\mathrm{~F}$ \\
\hline Soro & D629 & 68 & M \\
\hline Liquor/LCR & D630 & 59 & $M$ \\
\hline Liquor/LCR & D631 & 0 & $\mathrm{M}$ \\
\hline Soro & D632 & 76 & $M$ \\
\hline Soro & D633 & 68 & $\mathrm{~F}$ \\
\hline Soro & D634 & 38 & $M$ \\
\hline Soro & D635 & 12 & $\mathrm{M}$ \\
\hline Soro & D636 & 54 & $M$ \\
\hline Soro & D637 & 66 & $\mathrm{~F}$ \\
\hline Liquor/LCR & D638 & 35 & $F$ \\
\hline Soro & D639 & 50 & $F$ \\
\hline Soro & D640 & 58 & $\mathrm{M}$ \\
\hline Soro & D641 & 48 & M \\
\hline Soro & D642 & 53 & $\mathrm{M}$ \\
\hline Soro & D643 & 73 & $M$ \\
\hline Soro & D644 & 20 & $F$ \\
\hline Soro & D645 & 63 & $M$ \\
\hline Soro & D646 & 45 & $M$ \\
\hline Soro & D647 & 53 & $\mathrm{~F}$ \\
\hline Soro & D648 & 46 & $\mathrm{~F}$ \\
\hline
\end{tabular}




\begin{tabular}{|c|c|c|c|}
\hline Soro & D649 & 14 & $\mathrm{~F}$ \\
\hline Soro & D650 & 21 & $M$ \\
\hline Soro & D651 & 87 & $\mathrm{~F}$ \\
\hline Soro & D652 & 28 & $\mathrm{~F}$ \\
\hline Soro & D653 & 72 & $\mathrm{~F}$ \\
\hline Soro & D654 & 42 & $M$ \\
\hline Soro & D655 & 45 & $M$ \\
\hline Soro & D656 & 45 & $\mathrm{~F}$ \\
\hline Soro & D657 & 44 & $\mathrm{~F}$ \\
\hline Soro & D658 & 44 & $\mathrm{~F}$ \\
\hline Soro & D659 & 72 & $M$ \\
\hline Soro & D660 & 21 & $M$ \\
\hline Soro & D661 & 61 & $M$ \\
\hline Soro & D662 & 81 & $M$ \\
\hline Soro & D663 & 71 & $\mathrm{M}$ \\
\hline Soro & D664 & 73 & $\mathrm{~F}$ \\
\hline Soro & D665 & 27 & $\mathrm{~F}$ \\
\hline Soro & D666 & 27 & $M$ \\
\hline Soro & D667 & 46 & $\mathrm{~F}$ \\
\hline Soro & D668 & 76 & $M$ \\
\hline Soro & D669 & 3 & $M$ \\
\hline Soro & D670 & 81 & $\mathrm{~F}$ \\
\hline Soro & D671 & 64 & $\mathrm{M}$ \\
\hline Soro & D672 & 56 & $\mathrm{~F}$ \\
\hline Soro & D673 & 58 & $\mathrm{~F}$ \\
\hline Soro & D674 & 41 & $\mathrm{~F}$ \\
\hline Soro & D675 & 81 & $\mathrm{~F}$ \\
\hline Soro & D676 & 36 & $M$ \\
\hline Soro & D677 & 57 & $M$ \\
\hline Soro & D678 & 7 & $\mathrm{M}$ \\
\hline Soro & D679 & 44 & $M$ \\
\hline Soro & D680 & 77 & $M$ \\
\hline Soro & D681 & 56 & $M$ \\
\hline Soro & D682 & 52 & $\mathrm{~F}$ \\
\hline Soro & D683 & 74 & $M$ \\
\hline Soro & D684 & 52 & $M$ \\
\hline Soro & D685 & 38 & $\mathrm{~F}$ \\
\hline Soro & D686 & 81 & $\mathrm{~F}$ \\
\hline Soro & D687 & 52 & $M$ \\
\hline Soro & D688 & 72 & $M$ \\
\hline Soro & D689 & 60 & $\mathrm{~F}$ \\
\hline Soro & D690 & 41 & $\mathrm{~F}$ \\
\hline Soro & D691 & 76 & $M$ \\
\hline Soro & D692 & 24 & $\mathrm{~F}$ \\
\hline Soro & D693 & 33 & $M$ \\
\hline Soro & D694 & 41 & $\mathrm{M}$ \\
\hline Soro & D695 & 31 & $\mathrm{~F}$ \\
\hline Soro & D696 & 57 & $\mathrm{~F}$ \\
\hline Soro & D697 & 58 & M \\
\hline Soro & D698 & 72 & $M$ \\
\hline
\end{tabular}




\begin{tabular}{|c|c|c|c|}
\hline Soro & D699 & 18 & $M$ \\
\hline Soro & D700 & 41 & $M$ \\
\hline Soro & D701 & 57 & $\mathrm{~F}$ \\
\hline Liquor/LCR & D702 & 24 & $\mathrm{~F}$ \\
\hline Soro & D703 & 51 & $\mathrm{~F}$ \\
\hline Soro & D704 & 64 & $M$ \\
\hline Soro & D705 & 71 & M \\
\hline Soro & D706 & 59 & M \\
\hline Soro & D707 & 67 & $\mathrm{~F}$ \\
\hline Soro & D708 & 54 & $M$ \\
\hline Soro & D709 & 54 & $\mathrm{~F}$ \\
\hline Soro & D710 & 64 & $\mathrm{~F}$ \\
\hline Soro & D711 & 52 & $\mathrm{~F}$ \\
\hline Soro & D712 & 37 & $\mathrm{~F}$ \\
\hline Soro & D713 & 23 & $\mathrm{~F}$ \\
\hline Soro & D714 & 64 & $\mathrm{~F}$ \\
\hline Soro & D715 & 30 & $\mathrm{~F}$ \\
\hline Soro & D716 & 63 & $\mathrm{~F}$ \\
\hline Soro & D717 & 59 & M \\
\hline Liquor/LCR & D718 & 40 & $\mathrm{~F}$ \\
\hline Soro & D719 & 36 & M \\
\hline Soro & D720 & 61 & M \\
\hline Soro & D721 & 37 & M \\
\hline Soro & D722 & 42 & M \\
\hline Soro & D723 & 46 & M \\
\hline Soro & D724 & 77 & M \\
\hline Soro & D725 & 68 & M \\
\hline Soro & D726 & 80 & M \\
\hline Soro & D727 & 29 & $\mathrm{~F}$ \\
\hline Soro & D728 & 57 & M \\
\hline Soro & D729 & 56 & M \\
\hline Soro & D730 & 36 & $\mathrm{~F}$ \\
\hline Soro & D731 & 26 & $\mathrm{~F}$ \\
\hline Soro & D732 & 84 & M \\
\hline Soro & D733 & 72 & $\mathrm{~F}$ \\
\hline Soro & D734 & 52 & $M$ \\
\hline Soro & D735 & 57 & M \\
\hline Soro & D736 & 36 & M \\
\hline Soro & D737 & 53 & $M$ \\
\hline Soro & D738 & 53 & M \\
\hline Soro & D739 & 81 & M \\
\hline Soro & D740 & 63 & M \\
\hline Soro & D741 & 43 & M \\
\hline Soro & D742 & 71 & $\mathrm{~F}$ \\
\hline Soro & D743 & 6 & $\mathrm{~F}$ \\
\hline Soro & D744 & 7 & $\mathrm{~F}$ \\
\hline Soro & D745 & 69 & M \\
\hline Soro & D746 & 6 & M \\
\hline Soro & D747 & 86 & $\mathrm{~F}$ \\
\hline Soro & D748 & 82 & $\mathrm{~F}$ \\
\hline
\end{tabular}




\begin{tabular}{|c|c|c|c|}
\hline Soro & D749 & 15 & $\mathrm{~F}$ \\
\hline Soro & D750 & 32 & $\mathrm{~F}$ \\
\hline Soro & D751 & 68 & $\mathrm{~F}$ \\
\hline Soro & D752 & 49 & M \\
\hline Liquor/LCR & D753 & 77 & $\mathrm{~F}$ \\
\hline Soro & D754 & 40 & $M$ \\
\hline Soro & D755 & 45 & $\mathrm{~F}$ \\
\hline Soro & D756 & 81 & $\mathrm{~F}$ \\
\hline Soro & D757 & 58 & M \\
\hline Soro & D758 & 44 & $M$ \\
\hline Soro & D759 & 5 & $\mathrm{~F}$ \\
\hline Soro & D760 & 5 & M \\
\hline Soro & D761 & 4 & M \\
\hline Soro & D762 & 62 & M \\
\hline Soro & D763 & 51 & M \\
\hline Soro & D764 & 34 & $\mathrm{~F}$ \\
\hline Soro & D765 & 21 & $\mathrm{~F}$ \\
\hline Soro & D766 & 49 & $\mathrm{~F}$ \\
\hline Soro & D767 & 45 & $\mathrm{~F}$ \\
\hline Liquor/LCR & D768 & 67 & $\mathrm{~F}$ \\
\hline Soro & D769 & 67 & M \\
\hline Soro & D770 & 15 & $\mathrm{~F}$ \\
\hline Soro & D771 & 77 & M \\
\hline Soro & D772 & 18 & $\mathrm{~F}$ \\
\hline Soro & D773 & 61 & M \\
\hline Soro & D774 & 51 & M \\
\hline Soro & D775 & 58 & $\mathrm{~F}$ \\
\hline Soro & D776 & 49 & $\mathrm{~F}$ \\
\hline Soro & D777 & 43 & M \\
\hline Soro & D778 & 36 & M \\
\hline Soro & D779 & 15 & M \\
\hline Soro & D780 & 77 & $M$ \\
\hline Soro & D781 & 69 & M \\
\hline Soro & D782 & 66 & $\mathrm{~F}$ \\
\hline Soro & D783 & 58 & $\mathrm{~F}$ \\
\hline Soro & D784 & 55 & $M$ \\
\hline Soro & D785 & 37 & $\mathrm{~F}$ \\
\hline Soro & D786 & 45 & 1 \\
\hline Soro & D787 & 61 & $M$ \\
\hline Soro & D788 & 63 & M \\
\hline Soro & D789 & 81 & $\mathrm{~F}$ \\
\hline Soro & D790 & 56 & M \\
\hline Soro & D791 & 63 & $\mathrm{~F}$ \\
\hline Soro & D792 & 30 & $\mathrm{~F}$ \\
\hline Soro & D793 & 46 & $\mathrm{~F}$ \\
\hline Soro & D794 & 23 & $\mathrm{~F}$ \\
\hline Soro & D795 & 5 & M \\
\hline Soro & D796 & 58 & M \\
\hline Soro & D797 & 85 & $M$ \\
\hline Soro & D798 & 42 & $\mathrm{~F}$ \\
\hline
\end{tabular}




\begin{tabular}{|c|c|c|c|}
\hline Soro & D799 & 52 & M \\
\hline Soro & D800 & 9 & $\mathrm{~F}$ \\
\hline Soro & D801 & 19 & M \\
\hline Soro & D802 & 80 & M \\
\hline Soro & D803 & 42 & $\mathrm{~F}$ \\
\hline Soro & D804 & 36 & M \\
\hline Soro & D805 & 50 & $\mathrm{~F}$ \\
\hline Soro & D806 & 57 & $\mathrm{~F}$ \\
\hline Soro & D807 & 60 & $\mathrm{~F}$ \\
\hline Soro & D808 & 5 & M \\
\hline Soro & D809 & 33 & $\mathrm{~F}$ \\
\hline Soro & D810 & 52 & M \\
\hline Soro & D811 & 78 & M \\
\hline Soro & D812 & 45 & $\mathrm{M}$ \\
\hline Soro & D813 & 58 & $\mathrm{~F}$ \\
\hline Soro & D814 & 74 & $\mathrm{~F}$ \\
\hline Soro & D815 & 45 & M \\
\hline Soro & D816 & 34 & M \\
\hline Soro & D817 & 57 & $\mathrm{~F}$ \\
\hline Soro & D818 & 43 & $\mathrm{~F}$ \\
\hline Soro & D819 & 44 & M \\
\hline Soro & D820 & 72 & $\mathrm{~F}$ \\
\hline Soro & D821 & 67 & $\mathrm{~F}$ \\
\hline Soro & D822 & 54 & M \\
\hline Soro & D823 & 11 & M \\
\hline Liquor/LCR & D824 & 27 & M \\
\hline Soro & D825 & 25 & $\mathrm{~F}$ \\
\hline Soro & D826 & 42 & $\mathrm{~F}$ \\
\hline Soro & D827 & 13 & $\mathrm{~F}$ \\
\hline Soro & D828 & 89 & $\mathrm{~F}$ \\
\hline Liquor/LCR & D829 & 37 & $\mathrm{~F}$ \\
\hline Soro & D830 & 80 & M \\
\hline Soro & D831 & 48 & $\mathrm{~F}$ \\
\hline Soro & D832 & 23 & $\mathrm{~F}$ \\
\hline Soro & D833 & 47 & $\mathrm{~F}$ \\
\hline Soro & D834 & 62 & $\mathrm{~F}$ \\
\hline Soro & D835 & 54 & $\mathrm{~F}$ \\
\hline Soro & D836 & 47 & $\mathrm{~F}$ \\
\hline Soro & D837 & 71 & $\mathrm{~F}$ \\
\hline Soro & D838 & 69 & M \\
\hline Soro & D839 & 52 & M \\
\hline Soro & D840 & 59 & M \\
\hline Soro & D841 & 61 & $\mathrm{~F}$ \\
\hline Soro & D842 & 20 & $\mathrm{~F}$ \\
\hline Soro & D843 & 41 & M \\
\hline Soro & D844 & 48 & $M$ \\
\hline Soro & D845 & 58 & $\mathrm{~F}$ \\
\hline Soro & D846 & 52 & $M$ \\
\hline Soro & D847 & 11 & M \\
\hline Soro & D848 & 52 & M \\
\hline
\end{tabular}




\begin{tabular}{|c|c|c|c|}
\hline Soro & D849 & 59 & $\mathrm{~F}$ \\
\hline Soro & D850 & 86 & $M$ \\
\hline Soro & D851 & 72 & 1 \\
\hline Soro & D852 & 83 & $\mathrm{~F}$ \\
\hline Liquor/LCR & D853 & 78 & $\mathrm{~F}$ \\
\hline Soro & D854 & 47 & $\mathrm{~F}$ \\
\hline Soro & D855 & 41 & $\mathrm{~F}$ \\
\hline Soro & D856 & 51 & M \\
\hline Soro & D857 & 36 & M \\
\hline Soro & D858 & 58 & $M$ \\
\hline Soro & D859 & 52 & M \\
\hline Soro & D860 & 49 & $M$ \\
\hline Soro & D861 & 32 & $\mathrm{~F}$ \\
\hline Soro & D862 & 80 & $M$ \\
\hline Soro & D863 & 37 & M \\
\hline Soro & D864 & 43 & $\mathrm{~F}$ \\
\hline Soro & D865 & 55 & $\mathrm{~F}$ \\
\hline Soro & D866 & 63 & $\mathrm{~F}$ \\
\hline Soro & D867 & 49 & M \\
\hline Soro & D868 & 55 & M \\
\hline Soro & D869 & 45 & $\mathrm{~F}$ \\
\hline Soro & D870 & 45 & $\mathrm{~F}$ \\
\hline Soro & D871 & 79 & M \\
\hline Soro & D872 & 36 & $M$ \\
\hline Soro & D873 & 60 & $\mathrm{~F}$ \\
\hline Soro & D874 & 42 & $\mathrm{~F}$ \\
\hline Liquor/LCR & D875 & 57 & $M$ \\
\hline Soro & D876 & 54 & $M$ \\
\hline Soro & D877 & 64 & M \\
\hline Soro & D878 & 41 & $\mathrm{~F}$ \\
\hline Soro & D879 & 50 & $M$ \\
\hline Soro & D880 & 62 & $M$ \\
\hline Soro & D881 & 42 & $\mathrm{~F}$ \\
\hline Soro & D882 & 44 & $\mathrm{~F}$ \\
\hline Soro & D883 & 28 & $\mathrm{~F}$ \\
\hline Soro & D884 & 57 & $\mathrm{~F}$ \\
\hline Soro & D885 & 35 & $\mathrm{~F}$ \\
\hline Soro & D886 & 57 & $\mathrm{~F}$ \\
\hline Soro & D887 & 3 & $M$ \\
\hline Liquor/LCR & D888 & 35 & $\mathrm{~F}$ \\
\hline Soro & D889 & 84 & $\mathrm{~F}$ \\
\hline Soro & D890 & 35 & M \\
\hline Soro & D891 & 35 & $M$ \\
\hline Soro & D892 & 54 & $\mathrm{~F}$ \\
\hline Soro & D893 & 77 & $\mathrm{~F}$ \\
\hline Soro & D894 & 59 & $\mathrm{~F}$ \\
\hline Soro & D895 & 85 & $M$ \\
\hline Liquor/LCR & D896 & 25 & M \\
\hline Soro & D897 & 41 & $\mathrm{~F}$ \\
\hline Soro & D898 & 79 & $\mathrm{~F}$ \\
\hline
\end{tabular}




\begin{tabular}{|c|c|c|c|}
\hline Soro & D899 & 35 & $\mathrm{~F}$ \\
\hline Soro & D900 & 72 & $M$ \\
\hline Soro & D901 & 53 & $\mathrm{~F}$ \\
\hline Soro & D902 & 59 & M \\
\hline Soro & D903 & 36 & M \\
\hline Soro & D904 & 65 & $M$ \\
\hline Soro & D905 & 49 & $\mathrm{~F}$ \\
\hline Soro & D906 & 53 & $\mathrm{~F}$ \\
\hline Soro & D907 & 56 & $\mathrm{~F}$ \\
\hline Soro & D908 & 60 & $\mathrm{~F}$ \\
\hline Soro & D909 & 53 & $\mathrm{~F}$ \\
\hline Soro & D910 & 64 & $M$ \\
\hline Soro & D911 & 47 & $\mathrm{~F}$ \\
\hline Soro & D912 & 44 & $M$ \\
\hline Soro & D913 & 83 & $\mathrm{~F}$ \\
\hline Soro & D914 & 32 & $\mathrm{~F}$ \\
\hline Soro & D915 & 57 & $\mathrm{~F}$ \\
\hline Soro & D916 & 38 & M \\
\hline Soro & D917 & 43 & M \\
\hline Soro & D918 & 55 & $\mathrm{~F}$ \\
\hline Soro & D919 & 17 & $M$ \\
\hline Soro & D920 & 82 & $\mathrm{~F}$ \\
\hline Soro & D921 & 70 & M \\
\hline Soro & D922 & 30 & $M$ \\
\hline Soro & D923 & 44 & $\mathrm{~F}$ \\
\hline Soro & D924 & 71 & M \\
\hline Soro & D925 & 65 & $\mathrm{~F}$ \\
\hline Soro & D926 & 65 & $\mathrm{~F}$ \\
\hline Soro & D927 & 30 & $\mathrm{~F}$ \\
\hline Soro & D928 & 64 & $M$ \\
\hline Soro & D929 & 71 & $\mathrm{~F}$ \\
\hline Soro & D930 & 39 & $\mathrm{~F}$ \\
\hline Soro & D931 & 80 & $\mathrm{~F}$ \\
\hline Soro & D932 & 25 & $\mathrm{~F}$ \\
\hline Soro & D933 & 55 & M \\
\hline Soro & D934 & 48 & $\mathrm{~F}$ \\
\hline Soro & D935 & 56 & $\mathrm{~F}$ \\
\hline Liquor/LCR & D936 & 46 & $\mathrm{~F}$ \\
\hline Soro & D937 & 19 & $M$ \\
\hline Soro & D938 & 81 & $\mathrm{~F}$ \\
\hline Soro & D939 & 72 & $\mathrm{~F}$ \\
\hline Soro & D940 & 22 & $\mathrm{~F}$ \\
\hline Soro & D941 & 58 & $\mathrm{~F}$ \\
\hline Soro & D942 & 57 & $M$ \\
\hline Soro & D943 & 49 & $M$ \\
\hline Soro & D944 & 36 & $M$ \\
\hline Soro & D945 & 50 & $\mathrm{~F}$ \\
\hline Soro & D946 & 36 & $M$ \\
\hline Soro & D947 & 82 & $\mathrm{~F}$ \\
\hline Soro & D948 & 70 & $M$ \\
\hline
\end{tabular}




\begin{tabular}{|c|c|c|c|}
\hline Soro & D949 & 21 & M \\
\hline Soro & D950 & 56 & M \\
\hline Soro & D951 & 44 & M \\
\hline Soro & D952 & 14 & M \\
\hline Soro & D953 & 68 & $\mathrm{~F}$ \\
\hline Soro & D954 & 59 & $\mathrm{~F}$ \\
\hline Soro & D955 & 77 & $\mathrm{~F}$ \\
\hline Soro & D956 & 83 & M \\
\hline Soro & D957 & 20 & $\mathrm{~F}$ \\
\hline Soro & D958 & 63 & $\mathrm{~F}$ \\
\hline Soro & D959 & 39 & M \\
\hline Soro & D960 & 42 & $\mathrm{~F}$ \\
\hline Liquor/LCR & D961 & 46 & $\mathrm{~F}$ \\
\hline Soro & D962 & 18 & $\mathrm{M}$ \\
\hline Soro & D963 & 3 & $\mathrm{~F}$ \\
\hline Soro & D964 & 34 & $\mathrm{~F}$ \\
\hline Soro & D965 & 52 & $\mathrm{~F}$ \\
\hline Soro & D966 & 45 & $\mathrm{~F}$ \\
\hline Soro & D967 & 43 & M \\
\hline Soro & D968 & 57 & $\mathrm{~F}$ \\
\hline Soro & D969 & 71 & M \\
\hline Soro & D970 & 71 & M \\
\hline Soro & D971 & 42 & M \\
\hline Soro & D972 & 14 & $\mathrm{~F}$ \\
\hline Soro & D973 & 39 & $\mathrm{~F}$ \\
\hline Soro & D974 & 39 & $\mathrm{~F}$ \\
\hline Soro & D975 & 52 & $\mathrm{~F}$ \\
\hline Liquor/LCR & D976 & 72 & $\mathrm{~F}$ \\
\hline Soro & D977 & 20 & $\mathrm{~F}$ \\
\hline Soro & D978 & 16 & $\mathrm{M}$ \\
\hline Soro & D979 & 43 & $\mathrm{~F}$ \\
\hline Soro & D980 & 46 & $\mathrm{~F}$ \\
\hline Soro & D981 & 29 & $\mathrm{~F}$ \\
\hline Soro & D982 & 63 & M \\
\hline Soro & D983 & 32 & M \\
\hline Soro & D984 & 48 & $\mathrm{~F}$ \\
\hline Soro & D985 & 76 & $\mathrm{~F}$ \\
\hline Soro & D986 & 45 & M \\
\hline Soro & D987 & 25 & $\mathrm{~F}$ \\
\hline Soro & D988 & 54 & $\mathrm{~F}$ \\
\hline Soro & D989 & 56 & M \\
\hline Soro & D990 & 50 & $\mathrm{~F}$ \\
\hline Soro & D991 & 43 & $M$ \\
\hline Soro & D992 & 45 & M \\
\hline Soro & D993 & 17 & $\mathrm{~F}$ \\
\hline Soro & D994 & 62 & $\mathrm{~F}$ \\
\hline Soro & D995 & 36 & M \\
\hline Soro & D996 & 20 & $\mathrm{~F}$ \\
\hline Liquor/LCR & D997 & 40 & M \\
\hline Liquor/LCR & D998 & 40 & M \\
\hline
\end{tabular}




\begin{tabular}{|c|c|c|c|}
\hline Soro & D999 & 59 & $\mathrm{~F}$ \\
\hline Soro & D1000 & 57 & $M$ \\
\hline Soro & D1001 & 58 & $\mathrm{~F}$ \\
\hline Soro & D1002 & 84 & $M$ \\
\hline Soro & D1003 & 62 & $M$ \\
\hline Soro & D1004 & 53 & $\mathrm{~F}$ \\
\hline Soro & D1005 & 21 & $M$ \\
\hline Soro & D1006 & 81 & $\mathrm{~F}$ \\
\hline Soro & D1007 & 24 & $\mathrm{M}$ \\
\hline Soro & D1008 & 69 & $M$ \\
\hline Liquor/LCR & D1009 & 81 & $\mathrm{~F}$ \\
\hline Soro & D1010 & 53 & $M$ \\
\hline Soro & D1011 & 43 & $M$ \\
\hline Soro & D1012 & 27 & $M$ \\
\hline Soro & D1013 & 68 & $\mathrm{M}$ \\
\hline Soro & D1014 & 26 & $\mathrm{M}$ \\
\hline Soro & D1015 & 64 & $M$ \\
\hline Soro & D1016 & 60 & $M$ \\
\hline Liquor/LCR & D1017 & 74 & $M$ \\
\hline Soro & D1018 & 20 & $\mathrm{~F}$ \\
\hline Soro & D1019 & 29 & $\mathrm{~F}$ \\
\hline Soro & D1020 & 19 & $\mathrm{M}$ \\
\hline Soro & D1021 & 51 & $\mathrm{~F}$ \\
\hline Soro & D1022 & 59 & $M$ \\
\hline Soro & D1023 & 62 & $\mathrm{~F}$ \\
\hline Soro & D1024 & 22 & $\mathrm{~F}$ \\
\hline Soro & D1025 & 66 & M \\
\hline Soro & D1026 & 67 & $\mathrm{~F}$ \\
\hline Soro & D1027 & 40 & $\mathrm{~F}$ \\
\hline Soro & D1028 & 44 & $\mathrm{M}$ \\
\hline Soro & D1029 & 57 & $M$ \\
\hline Soro & D1030 & 64 & $\mathrm{M}$ \\
\hline Soro & D1031 & 24 & $M$ \\
\hline Soro & D1032 & 7 & $M$ \\
\hline Soro & D1033 & 55 & $M$ \\
\hline Soro & D1034 & 38 & $M$ \\
\hline Soro & D1035 & 82 & $\mathrm{M}$ \\
\hline Soro & D1036 & 79 & $M$ \\
\hline Soro & D1037 & 16 & $\mathrm{~F}$ \\
\hline Soro & D1038 & 57 & $M$ \\
\hline Soro & D1039 & 78 & $M$ \\
\hline Soro & D1040 & 16 & $\mathrm{~F}$ \\
\hline Soro & D1041 & 34 & $M$ \\
\hline Soro & D1042 & 45 & $\mathrm{~F}$ \\
\hline Soro & D1043 & 50 & $M$ \\
\hline Soro & D1044 & 50 & $M$ \\
\hline Soro & D1045 & 22 & $\mathrm{~F}$ \\
\hline Soro & D1046 & 62 & $M$ \\
\hline Soro & D1047 & 23 & $\mathrm{~F}$ \\
\hline Soro & D1048 & 33 & $M$ \\
\hline
\end{tabular}




\begin{tabular}{|c|c|c|c|}
\hline Soro & D1049 & 65 & $M$ \\
\hline Soro & D1050 & 57 & $M$ \\
\hline Liquor/LCR & D1051 & 61 & M \\
\hline Soro & D1052 & 26 & M \\
\hline Soro & D1053 & 62 & $\mathrm{~F}$ \\
\hline Soro & D1054 & 55 & $\mathrm{~F}$ \\
\hline Soro & D1055 & 20 & M \\
\hline Soro & D1056 & 24 & $\mathrm{~F}$ \\
\hline Soro & D1057 & 60 & M \\
\hline Soro & D1058 & 46 & $\mathrm{~F}$ \\
\hline Soro & D1059 & 58 & M \\
\hline Soro & D1060 & 45 & $\mathrm{~F}$ \\
\hline Soro & D1061 & 30 & $\mathrm{~F}$ \\
\hline Soro & D1062 & 52 & $\mathrm{M}$ \\
\hline Soro & D1063 & 75 & $\mathrm{~F}$ \\
\hline Soro & D1064 & 5 & M \\
\hline Soro & D1065 & 77 & $\mathrm{~F}$ \\
\hline Soro & D1066 & 6 & $\mathrm{~F}$ \\
\hline Soro & D1067 & 63 & M \\
\hline Soro & D1068 & 56 & M \\
\hline Soro & D1069 & 13 & M \\
\hline Soro & D1070 & 27 & $\mathrm{~F}$ \\
\hline Soro & D1071 & 49 & M \\
\hline Soro & D1072 & 66 & $\mathrm{~F}$ \\
\hline Soro & D1073 & 46 & M \\
\hline Soro & D1074 & 14 & M \\
\hline Soro & D1075 & 44 & M \\
\hline Soro & D1076 & 55 & M \\
\hline Soro & D1077 & 47 & M \\
\hline Soro & D1078 & 18 & $\mathrm{M}$ \\
\hline Soro & D1079 & 72 & $\mathrm{~F}$ \\
\hline Soro & D1080 & 13 & M \\
\hline Soro & D1081 & 49 & $\mathrm{~F}$ \\
\hline Soro & D1082 & 12 & M \\
\hline Soro & D1083 & 60 & M \\
\hline Liquor/LCR & D1084 & 45 & $\mathrm{~F}$ \\
\hline Soro & D1085 & 47 & $\mathrm{~F}$ \\
\hline Soro & D1086 & 57 & $\mathrm{~F}$ \\
\hline Soro & D1087 & 78 & M \\
\hline Soro & D1088 & 65 & M \\
\hline Soro & D1089 & 70 & $\mathrm{~F}$ \\
\hline Soro & D1090 & 55 & M \\
\hline Soro & D1091 & 47 & M \\
\hline Soro & D1092 & 13 & $\mathrm{~F}$ \\
\hline Soro & D1093 & 49 & M \\
\hline Soro & D1094 & 50 & $\mathrm{~F}$ \\
\hline Soro & D1095 & 29 & M \\
\hline Soro & D1096 & 79 & $\mathrm{~F}$ \\
\hline Soro & D1097 & 19 & $\mathrm{~F}$ \\
\hline Soro & D1098 & 73 & $\mathrm{~F}$ \\
\hline
\end{tabular}




\begin{tabular}{|c|c|c|c|}
\hline Soro & D1099 & 70 & M \\
\hline Soro & D1100 & 49 & M \\
\hline Soro & D1101 & 71 & M \\
\hline Soro & D1102 & 67 & M \\
\hline Soro & D1103 & 54 & M \\
\hline Soro & D1104 & 28 & $\mathrm{~F}$ \\
\hline Soro & D1105 & 40 & M \\
\hline Soro & D1106 & 53 & M \\
\hline Soro & D1107 & 27 & $\mathrm{~F}$ \\
\hline Soro & D1108 & 66 & M \\
\hline Soro & D1109 & 43 & $\mathrm{~F}$ \\
\hline Soro & D1110 & 33 & M \\
\hline Soro & D1111 & 35 & M \\
\hline Soro & D1112 & 36 & $\mathrm{~F}$ \\
\hline Soro & D1113 & 75 & M \\
\hline Soro & D1114 & 38 & M \\
\hline Soro & D1115 & 56 & $\mathrm{~F}$ \\
\hline Soro & D1116 & 38 & M \\
\hline Soro & D1117 & 34 & $\mathrm{~F}$ \\
\hline Soro & D1118 & 64 & $\mathrm{~F}$ \\
\hline Soro & D1119 & 72 & $\mathrm{~F}$ \\
\hline Soro & D1120 & 53 & M \\
\hline Soro & D1121 & 68 & $\mathrm{~F}$ \\
\hline Soro & D1122 & 48 & M \\
\hline Soro & D1123 & 65 & M \\
\hline Soro & D1124 & 52 & $\mathrm{~F}$ \\
\hline Soro & D1125 & 63 & M \\
\hline Soro & D1126 & 23 & $\mathrm{~F}$ \\
\hline Soro & D1127 & 55 & M \\
\hline Soro & D1128 & 42 & $\mathrm{~F}$ \\
\hline Soro & D1129 & 62 & $\mathrm{~F}$ \\
\hline Soro & D1130 & 64 & $\mathrm{~F}$ \\
\hline Soro & D1131 & 57 & M \\
\hline Soro & D1132 & 24 & $\mathrm{~F}$ \\
\hline Soro & D1133 & 54 & $\mathrm{~F}$ \\
\hline Liquor/LCR & D1134 & 32 & $M$ \\
\hline Soro & D1135 & 54 & M \\
\hline Soro & D1136 & 59 & M \\
\hline Soro & D1137 & 65 & M \\
\hline Soro & D1138 & 58 & $\mathrm{~F}$ \\
\hline Soro & D1139 & 33 & $\mathrm{~F}$ \\
\hline Soro & D1140 & 21 & $\mathrm{~F}$ \\
\hline Soro & D1141 & 68 & $M$ \\
\hline Soro & D1142 & 53 & $\mathrm{M}$ \\
\hline Soro & D1143 & 17 & M \\
\hline Soro & D1144 & 34 & $\mathrm{~F}$ \\
\hline Soro & D1145 & 41 & M \\
\hline Soro & D1146 & 33 & $M$ \\
\hline Soro & D1147 & 54 & $\mathrm{~F}$ \\
\hline Soro & D1148 & 85 & M \\
\hline
\end{tabular}




\begin{tabular}{|c|c|c|c|}
\hline Soro & D1149 & 56 & $M$ \\
\hline Soro & D1150 & 42 & $M$ \\
\hline Soro & D1151 & 35 & $\mathrm{~F}$ \\
\hline Soro & D1152 & 49 & $M$ \\
\hline Soro & D1153 & 50 & $\mathrm{~F}$ \\
\hline Soro & D1154 & 24 & M \\
\hline Soro & D1155 & 53 & $M$ \\
\hline Soro & D1156 & 51 & $\mathrm{~F}$ \\
\hline Soro & D1157 & 44 & $M$ \\
\hline Soro & D1158 & 76 & $M$ \\
\hline Soro & D1159 & 38 & $\mathrm{~F}$ \\
\hline Soro & D1160 & 39 & $\mathrm{~F}$ \\
\hline Soro & D1161 & 24 & M \\
\hline Soro & D1162 & 56 & $\mathrm{M}$ \\
\hline Soro & D1163 & 36 & $\mathrm{~F}$ \\
\hline Soro & D1164 & 6 & $\mathrm{~F}$ \\
\hline Soro & D1165 & 61 & $M$ \\
\hline Soro & D1166 & 63 & $M$ \\
\hline Soro & D1167 & 89 & $\mathrm{~F}$ \\
\hline Soro & D1168 & 36 & M \\
\hline Soro & D1169 & 57 & M \\
\hline Soro & D1170 & 52 & $\mathrm{~F}$ \\
\hline Soro & D1171 & 23 & M \\
\hline Soro & D1172 & 60 & $M$ \\
\hline Soro & D1173 & 49 & $M$ \\
\hline Soro & D1174 & 66 & $M$ \\
\hline Soro & D1175 & 52 & $\mathrm{~F}$ \\
\hline Soro & D1176 & 59 & M \\
\hline Soro & D1177 & 3 & $\mathrm{M}$ \\
\hline Soro & D1178 & 15 & $\mathrm{M}$ \\
\hline Soro & D1179 & 39 & $\mathrm{~F}$ \\
\hline Soro & D1180 & 21 & $\mathrm{~F}$ \\
\hline Soro & D1181 & 31 & $M$ \\
\hline Soro & D1182 & 51 & M \\
\hline Soro & D1183 & 29 & $\mathrm{~F}$ \\
\hline Soro & D1184 & 66 & $\mathrm{M}$ \\
\hline Soro & D1185 & 75 & $\mathrm{M}$ \\
\hline Soro & D1186 & 63 & $\mathrm{~F}$ \\
\hline Soro & D1187 & 63 & $\mathrm{~F}$ \\
\hline Soro & D1188 & 50 & $\mathrm{~F}$ \\
\hline Soro & D1189 & 23 & $\mathrm{~F}$ \\
\hline Soro & D1190 & 55 & $\mathrm{M}$ \\
\hline Soro & D1191 & 80 & $M$ \\
\hline Soro & D1192 & 60 & $\mathrm{M}$ \\
\hline Soro & D1193 & 52 & M \\
\hline Soro & D1194 & 45 & $M$ \\
\hline Soro & D1195 & 49 & $\mathrm{~F}$ \\
\hline Soro & D1196 & 49 & $\mathrm{~F}$ \\
\hline Soro & D1197 & 66 & $\mathrm{~F}$ \\
\hline Soro & D1198 & 20 & M \\
\hline
\end{tabular}




\begin{tabular}{|c|c|c|c|}
\hline Soro & D1199 & 51 & M \\
\hline Soro & D1200 & 55 & M \\
\hline Soro & D1201 & 77 & $\mathrm{~F}$ \\
\hline Soro & D1202 & 67 & $\mathrm{~F}$ \\
\hline Soro & D1203 & 65 & M \\
\hline Soro & D1204 & 54 & $\mathrm{~F}$ \\
\hline Soro & D1205 & 58 & M \\
\hline Soro & D1206 & 89 & M \\
\hline Soro & D1207 & 24 & $\mathrm{~F}$ \\
\hline Soro & D1208 & 50 & $\mathrm{~F}$ \\
\hline Soro & D1209 & 62 & M \\
\hline Soro & D1210 & 8 & M \\
\hline Soro & D1211 & 11 & M \\
\hline Soro & D1212 & 36 & M \\
\hline Soro & D1213 & 37 & $\mathrm{~F}$ \\
\hline Soro & D1214 & 39 & $\mathrm{~F}$ \\
\hline Liquor/LCR & D1215 & 65 & $\mathrm{~F}$ \\
\hline Soro & D1216 & 83 & $\mathrm{~F}$ \\
\hline Soro & D1217 & 59 & $\mathrm{~F}$ \\
\hline Soro & D1218 & 63 & M \\
\hline Soro & D1219 & 63 & M \\
\hline Soro & D1220 & 42 & $\mathrm{~F}$ \\
\hline Soro & D1221 & 82 & M \\
\hline Soro & D1222 & 61 & M \\
\hline Soro & D1223 & 84 & M \\
\hline Soro & D1224 & 55 & M \\
\hline Soro & D1225 & 34 & $\mathrm{~F}$ \\
\hline Soro & D1226 & 52 & M \\
\hline Soro & D1227 & 63 & $\mathrm{~F}$ \\
\hline Soro & D1228 & 42 & $\mathrm{M}$ \\
\hline Soro & D1229 & 31 & M \\
\hline Soro & D1230 & 83 & $\mathrm{~F}$ \\
\hline Soro & D1231 & 61 & M \\
\hline Soro & D1232 & 75 & $\mathrm{~F}$ \\
\hline Soro & D1233 & 62 & M \\
\hline Soro & D1234 & 16 & $\mathrm{~F}$ \\
\hline Soro & D1235 & 83 & $\mathrm{~F}$ \\
\hline Soro & D1236 & 65 & $\mathrm{~F}$ \\
\hline Liquor/LCR & D1237 & 82 & $\mathrm{~F}$ \\
\hline Soro & D1238 & 57 & M \\
\hline Soro & D1239 & 86 & $\mathrm{~F}$ \\
\hline Soro & D1240 & 39 & M \\
\hline Soro & D1241 & 52 & $\mathrm{~F}$ \\
\hline Soro & D1242 & 63 & M \\
\hline Soro & D1243 & 72 & $\mathrm{~F}$ \\
\hline Soro & D1244 & 44 & M \\
\hline Soro & D1245 & 70 & M \\
\hline Soro & D1246 & 56 & $\mathrm{~F}$ \\
\hline Liquor/LCR & D1247 & 47 & M \\
\hline Liquor/LCR & D1248 & 53 & $M$ \\
\hline
\end{tabular}




\begin{tabular}{|c|c|c|c|}
\hline Soro & D1249 & 59 & $\mathrm{~F}$ \\
\hline Soro & D1250 & 52 & $M$ \\
\hline Soro & D1251 & 42 & $M$ \\
\hline Soro & D1252 & 65 & M \\
\hline Soro & D1253 & 49 & M \\
\hline Soro & D1254 & 38 & $\mathrm{~F}$ \\
\hline Soro & D1255 & 87 & $\mathrm{~F}$ \\
\hline Soro & D1256 & 37 & M \\
\hline Soro & D1257 & 55 & $\mathrm{~F}$ \\
\hline Soro & D1258 & 73 & $\mathrm{~F}$ \\
\hline Soro & D1259 & 43 & M \\
\hline Soro & D1260 & 61 & $M$ \\
\hline Soro & D1261 & 48 & $\mathrm{~F}$ \\
\hline Soro & D1262 & 60 & $\mathrm{~F}$ \\
\hline Soro & D1263 & 78 & $\mathrm{~F}$ \\
\hline Soro & D1264 & 78 & $\mathrm{~F}$ \\
\hline Soro & D1265 & 54 & $M$ \\
\hline Soro & D1266 & 66 & M \\
\hline Soro & D1267 & 58 & $\mathrm{~F}$ \\
\hline Soro & D1268 & 74 & M \\
\hline Soro & D1269 & 41 & $M$ \\
\hline Liquor/LCR & D1270 & 30 & $\mathrm{~F}$ \\
\hline Soro & D1271 & 33 & $\mathrm{~F}$ \\
\hline Soro & D1272 & 38 & $\mathrm{~F}$ \\
\hline Soro & D1273 & 40 & $\mathrm{~F}$ \\
\hline Soro & D1274 & 39 & M \\
\hline Liquor/LCR & D1275 & 75 & $M$ \\
\hline Soro & D1276 & 64 & M \\
\hline Liquor/LCR & D1277 & 33 & $M$ \\
\hline Liquor/LCR & D1278 & 45 & $M$ \\
\hline Liquor/LCR & D1279 & 41 & $\mathrm{~F}$ \\
\hline Soro & D1280 & 57 & M \\
\hline Soro & D1281 & 26 & $M$ \\
\hline Soro & D1282 & 61 & $\mathrm{~F}$ \\
\hline Soro & D1283 & 49 & $\mathrm{~F}$ \\
\hline Soro & D1284 & 67 & $\mathrm{~F}$ \\
\hline Soro & D1285 & 49 & $\mathrm{~F}$ \\
\hline Soro & D1286 & 64 & $\mathrm{~F}$ \\
\hline Liquor/LCR & D1287 & 42 & M \\
\hline Liquor/LCR & D1288 & 50 & $M$ \\
\hline Liquor/LCR & D1289 & 50 & M \\
\hline Liquor/LCR & D1290 & 74 & $\mathrm{~F}$ \\
\hline Soro & D1291 & 54 & $M$ \\
\hline Soro & D1292 & 68 & $\mathrm{~F}$ \\
\hline Liquor/LCR & D1293 & 44 & $\mathrm{~F}$ \\
\hline Soro & D1294 & 86 & $M$ \\
\hline Soro & D1295 & 53 & $M$ \\
\hline Soro & D1296 & 52 & $\mathrm{~F}$ \\
\hline Soro & D1297 & 81 & M \\
\hline Soro & D1298 & 15 & $M$ \\
\hline
\end{tabular}




\begin{tabular}{|c|c|c|c|}
\hline Soro & D1299 & 20 & $\mathrm{~F}$ \\
\hline Soro & D1300 & 75 & $\mathrm{~F}$ \\
\hline Soro & D1301 & 25 & M \\
\hline Soro & D1302 & 79 & $\mathrm{~F}$ \\
\hline Soro & D1303 & 60 & $\mathrm{~F}$ \\
\hline Soro & D1304 & 55 & $M$ \\
\hline Soro & D1305 & 31 & M \\
\hline Soro & D1306 & 33 & $\mathrm{~F}$ \\
\hline Soro & D1307 & 39 & $\mathrm{~F}$ \\
\hline Soro & D1308 & 7 & $M$ \\
\hline Soro & D1309 & 82 & $\mathrm{~F}$ \\
\hline Soro & D1310 & 70 & $\mathrm{~F}$ \\
\hline Soro & D1311 & 34 & M \\
\hline Soro & D1312 & 81 & M \\
\hline Soro & D1313 & 30 & M \\
\hline Soro & D1314 & 39 & M \\
\hline Soro & D1315 & 62 & $\mathrm{~F}$ \\
\hline Soro & D1316 & 65 & $\mathrm{~F}$ \\
\hline Soro & D1317 & 42 & M \\
\hline Soro & D1318 & 35 & M \\
\hline Soro & D1319 & 70 & $\mathrm{~F}$ \\
\hline Soro & D1320 & 69 & M \\
\hline Soro & D1321 & 59 & $\mathrm{~F}$ \\
\hline Soro & D1322 & 64 & M \\
\hline Soro & D1323 & 83 & M \\
\hline Soro & D1324 & 33 & $\mathrm{~F}$ \\
\hline Liquor/LCR & D1325 & 84 & $\mathrm{~F}$ \\
\hline Liquor/LCR & D1326 & 84 & M \\
\hline Soro & D1327 & 25 & M \\
\hline Soro & D1328 & 71 & $\mathrm{~F}$ \\
\hline Soro & D1329 & 38 & $\mathrm{~F}$ \\
\hline Soro & D1330 & 74 & $M$ \\
\hline Soro & D1331 & 66 & M \\
\hline Soro & D1332 & 78 & M \\
\hline Liquor/LCR & D1333 & 84 & M \\
\hline Soro & D1334 & 81 & $\mathrm{~F}$ \\
\hline Soro & D1335 & 71 & M \\
\hline Soro & D1336 & 29 & M \\
\hline Liquor/LCR & D1337 & 41 & $M$ \\
\hline Soro & D1338 & 42 & $\mathrm{~F}$ \\
\hline Soro & D1339 & 71 & $\mathrm{~F}$ \\
\hline Soro & D1340 & 84 & M \\
\hline Soro & D1341 & 78 & $\mathrm{~F}$ \\
\hline Soro & D1342 & 78 & M \\
\hline Soro & D1343 & 71 & M \\
\hline Liquor/LCR & D1344 & 70 & $M$ \\
\hline Soro & D1345 & 75 & $\mathrm{~F}$ \\
\hline Soro & D1346 & 72 & $\mathrm{~F}$ \\
\hline Soro & D1347 & 23 & $\mathrm{~F}$ \\
\hline Soro & D1348 & 73 & M \\
\hline
\end{tabular}




\begin{tabular}{|c|c|c|c|}
\hline Soro & D1349 & 44 & $F$ \\
\hline Soro & D1350 & 24 & $\mathrm{~F}$ \\
\hline Soro & D1351 & 35 & $M$ \\
\hline Soro & D1352 & 44 & $F$ \\
\hline Soro & D1353 & 44 & $F$ \\
\hline Soro & D1354 & 92 & $M$ \\
\hline Soro & D1355 & 55 & $F$ \\
\hline Soro & D1356 & 86 & $F$ \\
\hline Soro & D1357 & 44 & $\mathrm{M}$ \\
\hline Soro & D1358 & 37 & $F$ \\
\hline Soro & D1359 & 67 & $M$ \\
\hline Soro & D1360 & 44 & $F$ \\
\hline Soro & D1361 & 49 & $F$ \\
\hline Soro & D1362 & 69 & $\mathrm{~F}$ \\
\hline Soro & D1363 & 33 & I \\
\hline Liquor/LCR & D1364 & 31 & $F$ \\
\hline Soro & D1365 & 51 & $\mathrm{M}$ \\
\hline Soro & D1366 & 38 & $F$ \\
\hline Soro & D1367 & 74 & $F$ \\
\hline Soro & D1368 & 82 & $F$ \\
\hline Soro & D1369 & 66 & M \\
\hline Soro & D1370 & 80 & $\mathrm{M}$ \\
\hline Soro & D1371 & 61 & $\mathrm{M}$ \\
\hline Soro & D1372 & 47 & $M$ \\
\hline Soro & D1373 & 55 & $F$ \\
\hline Soro & D1374 & 55 & $\mathrm{M}$ \\
\hline Soro & D1375 & 32 & $M$ \\
\hline Soro & D1376 & 73 & $\mathrm{M}$ \\
\hline Soro & D1377 & 74 & $M$ \\
\hline Liquor/LCR & D1378 & 50 & $\mathrm{M}$ \\
\hline Soro & D1379 & 53 & M \\
\hline Soro & D1380 & 66 & $M$ \\
\hline Soro & D1381 & 87 & $F$ \\
\hline Soro & D1382 & 91 & $F$ \\
\hline Soro & D1383 & 37 & I \\
\hline Soro & D1384 & 90 & $\mathrm{~F}$ \\
\hline Soro & D1385 & 80 & $F$ \\
\hline Soro & D1386 & 85 & $M$ \\
\hline Soro & D1387 & 54 & $F$ \\
\hline Soro & D1388 & 25 & $\mathrm{M}$ \\
\hline Soro & D1389 & 83 & $M$ \\
\hline Soro & D1390 & 83 & $\mathrm{M}$ \\
\hline Soro & D1391 & 11 & $\mathrm{~F}$ \\
\hline Soro & D1392 & 52 & $F$ \\
\hline Soro & D1393 & 90 & $M$ \\
\hline Soro & D1394 & 49 & $F$ \\
\hline Soro & D1395 & 28 & $M$ \\
\hline Soro & D1396 & 71 & $\mathrm{M}$ \\
\hline Soro & D1397 & 72 & $F$ \\
\hline Soro & D1398 & 77 & $\mathrm{M}$ \\
\hline
\end{tabular}




\begin{tabular}{|c|c|c|c|}
\hline Liquor/LCR & D1399 & 52 & $\mathrm{~F}$ \\
\hline Soro & D1400 & 35 & $\mathrm{~F}$ \\
\hline Soro & D1401 & 47 & $M$ \\
\hline Soro & D1402 & 55 & $M$ \\
\hline Liquor/LCR & D1403 & 56 & $M$ \\
\hline Soro & D1404 & 54 & $\mathrm{~F}$ \\
\hline Soro & D1405 & 19 & $\mathrm{~F}$ \\
\hline Soro & D1406 & 51 & $M$ \\
\hline Soro & D1407 & 24 & $F$ \\
\hline Soro & D1408 & 54 & $\mathrm{~F}$ \\
\hline Soro & D1409 & 60 & $\mathrm{~F}$ \\
\hline Soro & D1410 & 51 & $\mathrm{~F}$ \\
\hline Soro & D1411 & 37 & $\mathrm{~F}$ \\
\hline Soro & D1412 & 79 & $\mathrm{~F}$ \\
\hline Soro & D1413 & 44 & $\mathrm{M}$ \\
\hline Soro & D1414 & 64 & $\mathrm{~F}$ \\
\hline Soro & D1415 & 72 & $\mathrm{~F}$ \\
\hline Soro & D1416 & 67 & $\mathrm{~F}$ \\
\hline Soro & D1417 & 70 & $M$ \\
\hline Soro & D1418 & 60 & $\mathrm{~F}$ \\
\hline Soro & D1419 & 42 & $\mathrm{~F}$ \\
\hline Soro & D1420 & 39 & $\mathrm{~F}$ \\
\hline Soro & D1421 & 71 & $M$ \\
\hline Soro & D1422 & 73 & $M$ \\
\hline Soro & D1423 & 40 & $M$ \\
\hline Soro & D1424 & 28 & $\mathrm{~F}$ \\
\hline Soro & D1425 & 42 & $\mathrm{~F}$ \\
\hline Soro & D1426 & 43 & $\mathrm{M}$ \\
\hline Soro & D1427 & 69 & $\mathrm{~F}$ \\
\hline Soro & D1428 & 49 & $\mathrm{M}$ \\
\hline Liquor/LCR & D1429 & 49 & $\mathrm{~F}$ \\
\hline Soro & D1430 & 33 & $\mathrm{~F}$ \\
\hline Liquor/LCR & D1431 & 85 & $\mathrm{~F}$ \\
\hline Soro & D1432 & 88 & $\mathrm{~F}$ \\
\hline Soro & D1433 & 69 & $\mathrm{~F}$ \\
\hline Soro & D1434 & 55 & $\mathrm{~F}$ \\
\hline Soro & D1435 & 76 & $\mathrm{M}$ \\
\hline Soro & D1436 & 59 & $\mathrm{~F}$ \\
\hline Liquor/LCR & D1437 & 40 & $M$ \\
\hline Soro & D1438 & 66 & $M$ \\
\hline Soro & D1439 & 84 & $M$ \\
\hline Soro & D1440 & 75 & $M$ \\
\hline Soro & D1441 & 33 & $\mathrm{~F}$ \\
\hline Soro & D1442 & 45 & $\mathrm{~F}$ \\
\hline Liquor/LCR & D1443 & 40 & $\mathrm{~F}$ \\
\hline Soro & D1444 & 67 & $\mathrm{M}$ \\
\hline Soro & D1445 & 35 & $M$ \\
\hline Soro & D1446 & 51 & $M$ \\
\hline Soro & D1447 & 66 & $\mathrm{~F}$ \\
\hline Soro & D1448 & 28 & $\mathrm{~F}$ \\
\hline
\end{tabular}




\begin{tabular}{|c|c|c|c|}
\hline Soro & D1449 & 63 & $\mathrm{~F}$ \\
\hline Soro & D1450 & 61 & $M$ \\
\hline Soro & D1451 & 55 & $\mathrm{~F}$ \\
\hline Soro & D1452 & 48 & $\mathrm{~F}$ \\
\hline Soro & D1453 & 35 & $\mathrm{~F}$ \\
\hline Soro & D1454 & 0 & $M$ \\
\hline Soro & D1455 & 0 & M \\
\hline Soro & D1456 & 40 & M \\
\hline Soro & D1457 & 47 & M \\
\hline Liquor/LCR & D1458 & 92 & $M$ \\
\hline Soro & D1459 & 11 & $\mathrm{~F}$ \\
\hline Liquor/LCR & D1460 & 87 & M \\
\hline Soro & D1461 & 83 & $\mathrm{~F}$ \\
\hline Liquor/LCR & D1462 & 30 & $\mathrm{~F}$ \\
\hline Soro & D1463 & 46 & M \\
\hline Soro & D1464 & 80 & $\mathrm{~F}$ \\
\hline Soro & D1465 & 54 & $\mathrm{~F}$ \\
\hline Soro & D1466 & 29 & M \\
\hline Soro & D1467 & 25 & M \\
\hline Soro & D1468 & 25 & M \\
\hline Soro & D1469 & 48 & $\mathrm{~F}$ \\
\hline Soro & D1470 & 11 & M \\
\hline Soro & D1471 & 82 & $\mathrm{~F}$ \\
\hline Soro & D1472 & 32 & $\mathrm{~F}$ \\
\hline Soro & D1473 & 56 & M \\
\hline Soro & D1474 & 85 & $\mathrm{~F}$ \\
\hline Soro & D1475 & 39 & M \\
\hline Soro & D1476 & 85 & M \\
\hline Soro & D1477 & 52 & M \\
\hline Soro & D1478 & 50 & $\mathrm{~F}$ \\
\hline Soro & D1479 & 47 & $\mathrm{~F}$ \\
\hline Soro & D1480 & 36 & $\mathrm{~F}$ \\
\hline Soro & D1481 & 59 & $\mathrm{~F}$ \\
\hline Soro & D1482 & 31 & $\mathrm{~F}$ \\
\hline Liquor/LCR & D1483 & 51 & M \\
\hline Liquor/LCR & D1484 & 53 & $\mathrm{~F}$ \\
\hline Soro & D1485 & 62 & $\mathrm{~F}$ \\
\hline Soro & D1486 & 53 & $\mathrm{~F}$ \\
\hline Soro & D1487 & 36 & $M$ \\
\hline Soro & D1488 & 51 & $\mathrm{~F}$ \\
\hline Soro & D1489 & 62 & $\mathrm{~F}$ \\
\hline Soro & D1490 & 63 & $\mathrm{~F}$ \\
\hline Soro & D1491 & 78 & $\mathrm{~F}$ \\
\hline Soro & D1492 & 0 & $\mathrm{~F}$ \\
\hline Soro & D1493 & 77 & $\mathrm{~F}$ \\
\hline Soro & D1494 & 35 & $\mathrm{~F}$ \\
\hline Liquor/LCR & D1495 & 63 & $\mathrm{~F}$ \\
\hline Soro & D1496 & 69 & $\mathrm{~F}$ \\
\hline Soro & D1497 & 49 & M \\
\hline Soro & D1498 & 66 & $\mathrm{~F}$ \\
\hline
\end{tabular}




\begin{tabular}{|c|c|c|c|}
\hline Soro & D1499 & 49 & $\mathrm{~F}$ \\
\hline Soro & D1500 & 85 & $M$ \\
\hline Soro & D1501 & 0 & M \\
\hline Soro & D1502 & 36 & $\mathrm{~F}$ \\
\hline Soro & D1503 & 63 & $\mathrm{~F}$ \\
\hline Soro & D1504 & 71 & $\mathrm{~F}$ \\
\hline Soro & D1505 & 75 & $\mathrm{~F}$ \\
\hline Soro & D1506 & 68 & M \\
\hline Soro & D1507 & 47 & $\mathrm{~F}$ \\
\hline Soro & D1508 & 81 & $M$ \\
\hline Soro & D1509 & 37 & $\mathrm{~F}$ \\
\hline Soro & D1510 & 0 & $\mathrm{~F}$ \\
\hline Soro & D1511 & 91 & $M$ \\
\hline Soro & D1512 & 36 & M \\
\hline Soro & D1513 & 85 & M \\
\hline Soro & D1514 & 49 & $\mathrm{~F}$ \\
\hline Soro & D1515 & 45 & $\mathrm{~F}$ \\
\hline Soro & D1516 & 65 & $\mathrm{~F}$ \\
\hline Soro & D1517 & 61 & M \\
\hline Soro & D1518 & 36 & $\mathrm{~F}$ \\
\hline Soro & D1519 & 35 & M \\
\hline Soro & D1520 & 29 & M \\
\hline Soro & D1521 & 66 & $\mathrm{~F}$ \\
\hline Soro & D1522 & 51 & $\mathrm{~F}$ \\
\hline Soro & D1523 & 72 & $\mathrm{~F}$ \\
\hline Soro & D1524 & 32 & $\mathrm{~F}$ \\
\hline Soro & D1525 & 33 & $\mathrm{~F}$ \\
\hline Soro & D1526 & 62 & $\mathrm{~F}$ \\
\hline Soro & D1527 & 0 & M \\
\hline Liquor/LCR & D1528 & 63 & M \\
\hline Soro & D1529 & 48 & M \\
\hline Soro & D1530 & 74 & $M$ \\
\hline Soro & D1531 & 45 & $\mathrm{~F}$ \\
\hline Soro & D1532 & 48 & M \\
\hline Liquor/LCR & D1533 & 55 & M \\
\hline Soro & D1534 & 12 & $\mathrm{~F}$ \\
\hline Soro & D1535 & 75 & $\mathrm{~F}$ \\
\hline Soro & D1536 & 56 & M \\
\hline Soro & D1537 & 70 & $\mathrm{~F}$ \\
\hline Soro & D1538 & 34 & $\mathrm{~F}$ \\
\hline Soro & D1539 & 38 & M \\
\hline Soro & D1540 & 76 & M \\
\hline Soro & D1541 & 20 & $\mathrm{~F}$ \\
\hline Soro & D1542 & 83 & $\mathrm{~F}$ \\
\hline Soro & D1543 & 72 & $\mathrm{~F}$ \\
\hline Soro & D1544 & 24 & $\mathrm{M}$ \\
\hline Soro & D1545 & 63 & $\mathrm{~F}$ \\
\hline Soro & D1546 & 34 & $\mathrm{~F}$ \\
\hline Soro & D1547 & 83 & $\mathrm{~F}$ \\
\hline Soro & D1548 & 45 & $\mathrm{~F}$ \\
\hline
\end{tabular}




\begin{tabular}{|c|c|c|c|}
\hline Soro & D1549 & 79 & $M$ \\
\hline Soro & D1550 & 0 & $M$ \\
\hline Soro & D1551 & 0 & M \\
\hline Soro & D1552 & 69 & M \\
\hline Soro & D1553 & 56 & M \\
\hline Soro & D1554 & 9 & $M$ \\
\hline Soro & D1555 & 63 & $\mathrm{~F}$ \\
\hline Soro & D1556 & 75 & M \\
\hline Soro & D1557 & 78 & M \\
\hline Soro & D1558 & 56 & $M$ \\
\hline Soro & D1559 & 50 & M \\
\hline Soro & D1560 & 63 & $\mathrm{~F}$ \\
\hline Soro & D1561 & 61 & $\mathrm{~F}$ \\
\hline Soro & D1562 & 89 & M \\
\hline Soro & D1563 & 83 & $\mathrm{~F}$ \\
\hline Soro & D1564 & 57 & $\mathrm{~F}$ \\
\hline Soro & D1565 & 46 & $\mathrm{~F}$ \\
\hline Soro & D1566 & 46 & $\mathrm{~F}$ \\
\hline Soro & D1567 & 93 & $\mathrm{~F}$ \\
\hline Soro & D1568 & 9 & $\mathrm{~F}$ \\
\hline Soro & D1569 & 0 & $\mathrm{~F}$ \\
\hline Soro & D1570 & 0 & M \\
\hline Soro & D1571 & 89 & $\mathrm{~F}$ \\
\hline Soro & D1572 & 89 & M \\
\hline Soro & D1573 & 23 & $\mathrm{~F}$ \\
\hline Soro & D1574 & 75 & $\mathrm{~F}$ \\
\hline Soro & D1575 & 10 & M \\
\hline Soro & D1576 & 22 & $\mathrm{~F}$ \\
\hline Soro & D1577 & 0 & $\mathrm{~F}$ \\
\hline Soro & D1578 & 58 & $\mathrm{~F}$ \\
\hline Soro & D1579 & 50 & M \\
\hline Soro & D1580 & 7 & $M$ \\
\hline Soro & D1581 & 48 & M \\
\hline Soro & D1582 & 0 & M \\
\hline Soro & D1583 & 72 & M \\
\hline Soro & D1584 & 53 & $\mathrm{~F}$ \\
\hline Soro & D1585 & 53 & $\mathrm{M}$ \\
\hline Soro & D1586 & 53 & $\mathrm{~F}$ \\
\hline Soro & D1587 & 46 & $M$ \\
\hline Liquor/LCR & D1588 & 55 & M \\
\hline Soro & D1589 & 60 & $\mathrm{~F}$ \\
\hline Soro & D1590 & 38 & $\mathrm{~F}$ \\
\hline Soro & D1591 & 55 & $\mathrm{~F}$ \\
\hline Soro & D1592 & 44 & $\mathrm{M}$ \\
\hline Soro & D1593 & 21 & $\mathrm{~F}$ \\
\hline Soro & D1594 & 55 & M \\
\hline Soro & D1595 & 45 & $\mathrm{~F}$ \\
\hline Soro & D1596 & 56 & M \\
\hline Soro & D1597 & 31 & M \\
\hline Soro & D1598 & 42 & M \\
\hline
\end{tabular}




\begin{tabular}{|c|c|c|c|}
\hline Soro & D1599 & 70 & $\mathrm{~F}$ \\
\hline Soro & D1600 & 39 & $\mathrm{~F}$ \\
\hline Soro & D1601 & 42 & $\mathrm{~F}$ \\
\hline Soro & D1602 & 0 & 1 \\
\hline Soro & D1603 & 0 & I \\
\hline Soro & D1604 & 0 & I \\
\hline Soro & D1605 & 0 & M \\
\hline Soro & D1606 & 0 & $\mathrm{~F}$ \\
\hline Soro & D1607 & 56 & $\mathrm{~F}$ \\
\hline Soro & D1608 & 70 & $M$ \\
\hline Soro & D1609 & 73 & $\mathrm{~F}$ \\
\hline Soro & D1610 & 56 & M \\
\hline Soro & D1611 & 44 & M \\
\hline Soro & D1612 & 49 & M \\
\hline Soro & D1613 & 0 & M \\
\hline Soro & D1614 & 0 & M \\
\hline Soro & D1615 & 19 & $\mathrm{~F}$ \\
\hline Soro & D1616 & 6 & M \\
\hline Liquor/LCR & D1617 & 77 & M \\
\hline Soro & D1618 & 54 & M \\
\hline Liquor/LCR & D1619 & 0 & $\mathrm{~F}$ \\
\hline Soro & D1620 & 0 & M \\
\hline Soro & D1621 & 0 & M \\
\hline Soro & D1622 & 49 & $\mathrm{~F}$ \\
\hline Soro & D1623 & 40 & M \\
\hline Soro & D1624 & 46 & $\mathrm{~F}$ \\
\hline Soro & D1625 & 61 & $\mathrm{~F}$ \\
\hline Soro & D1626 & 45 & M \\
\hline Soro & D1627 & 40 & $\mathrm{~F}$ \\
\hline Soro & D1628 & 95 & $\mathrm{~F}$ \\
\hline Soro & D1629 & 67 & M \\
\hline Soro & D1630 & 58 & $M$ \\
\hline Soro & D1631 & 41 & M \\
\hline Soro & D1632 & 14 & $\mathrm{~F}$ \\
\hline Soro & D1633 & 88 & M \\
\hline Soro & D1634 & 0 & M \\
\hline Soro & D1635 & 40 & 1 \\
\hline Soro & D1636 & 73 & M \\
\hline Soro & D1637 & 0 & $\mathrm{~F}$ \\
\hline Soro & D1638 & 56 & $\mathrm{~F}$ \\
\hline Soro & D1639 & 54 & $\mathrm{~F}$ \\
\hline Soro & D1640 & 75 & M \\
\hline Soro & D1641 & 53 & M \\
\hline Soro & D1642 & 82 & M \\
\hline Soro & D1643 & 71 & M \\
\hline Soro & D1644 & 39 & M \\
\hline Soro & D1645 & 41 & M \\
\hline Soro & D1646 & 28 & M \\
\hline Soro & D1647 & 54 & $\mathrm{~F}$ \\
\hline Soro & D1648 & 63 & $\mathrm{~F}$ \\
\hline
\end{tabular}




\begin{tabular}{|c|c|c|c|}
\hline Soro & D1649 & 53 & $\mathrm{~F}$ \\
\hline Soro & D1650 & 78 & $M$ \\
\hline Soro & D1651 & 40 & M \\
\hline Soro & D1652 & 62 & $\mathrm{~F}$ \\
\hline Soro & D1653 & 57 & M \\
\hline Soro & D1654 & 59 & $\mathrm{~F}$ \\
\hline Liquor/LCR & D1655 & 85 & $\mathrm{~F}$ \\
\hline Soro & D1656 & 70 & M \\
\hline Soro & D1657 & 47 & M \\
\hline Soro & D1658 & 11 & $M$ \\
\hline Soro & D1659 & 0 & M \\
\hline Soro & D1660 & 0 & $\mathrm{~F}$ \\
\hline Soro & D1661 & 44 & $\mathrm{~F}$ \\
\hline Soro & D1662 & 76 & $\mathrm{~F}$ \\
\hline Soro & D1663 & 65 & $\mathrm{~F}$ \\
\hline Soro & D1664 & 44 & $M$ \\
\hline Soro & D1665 & 39 & $\mathrm{~F}$ \\
\hline Soro & D1666 & 30 & $\mathrm{~F}$ \\
\hline Soro & D1667 & 53 & $\mathrm{~F}$ \\
\hline Soro & D1668 & 42 & M \\
\hline Soro & D1669 & 70 & M \\
\hline Soro & D1670 & 71 & M \\
\hline Soro & D1671 & 55 & M \\
\hline Soro & D1672 & 61 & M \\
\hline Soro & D1673 & 57 & M \\
\hline Soro & D1674 & 62 & $\mathrm{~F}$ \\
\hline Soro & D1675 & 82 & $\mathrm{~F}$ \\
\hline Soro & D1676 & 59 & $\mathrm{~F}$ \\
\hline Soro & D1677 & 0 & M \\
\hline Soro & D1678 & 0 & $\mathrm{~F}$ \\
\hline Soro & D1679 & 0 & M \\
\hline Soro & D1680 & 74 & $\mathrm{~F}$ \\
\hline Soro & D1681 & 0 & $\mathrm{~F}$ \\
\hline Soro & D1682 & 0 & $\mathrm{~F}$ \\
\hline Soro & D1683 & 44 & M \\
\hline Soro & D1684 & 21 & $\mathrm{~F}$ \\
\hline Soro & D1685 & 74 & $\mathrm{M}$ \\
\hline Soro & D1686 & 0 & M \\
\hline Soro & D1687 & 44 & $M$ \\
\hline Soro & D1688 & 59 & $\mathrm{~F}$ \\
\hline Soro & D1689 & 63 & M \\
\hline Soro & D1690 & 76 & $\mathrm{~F}$ \\
\hline Soro & D1691 & 60 & $\mathrm{~F}$ \\
\hline Soro & D1692 & 24 & $\mathrm{~F}$ \\
\hline Soro & D1693 & 61 & M \\
\hline Soro & D1694 & 32 & $\mathrm{~F}$ \\
\hline Soro & D1695 & 70 & M \\
\hline Soro & D1696 & 31 & $\mathrm{~F}$ \\
\hline Soro & D1697 & 77 & M \\
\hline Soro & D1698 & 0 & $\mathrm{~F}$ \\
\hline
\end{tabular}




\begin{tabular}{|c|c|c|c|}
\hline Soro & D1699 & 39 & $\mathrm{~F}$ \\
\hline Soro & D1700 & 50 & $\mathrm{~F}$ \\
\hline Soro & D1701 & 38 & $M$ \\
\hline Soro & D1702 & 56 & M \\
\hline Soro & D1703 & 73 & M \\
\hline Liquor/LCR & D1704 & 44 & M \\
\hline Soro & D1705 & 37 & $M$ \\
\hline Soro & D1706 & 54 & M \\
\hline Soro & D1707 & 59 & M \\
\hline Soro & D1708 & 90 & $\mathrm{~F}$ \\
\hline Soro & D1709 & 47 & $\mathrm{~F}$ \\
\hline Soro & D1710 & 49 & $M$ \\
\hline Soro & D1711 & 73 & $M$ \\
\hline Soro & D1712 & 34 & $\mathrm{~F}$ \\
\hline Soro & D1713 & 7 & M \\
\hline Soro & D1714 & 51 & M \\
\hline Soro & D1715 & 44 & $M$ \\
\hline Soro & D1716 & 25 & $\mathrm{~F}$ \\
\hline Soro & D1717 & 44 & M \\
\hline Soro & D1718 & 71 & $\mathrm{~F}$ \\
\hline Soro & D1719 & 69 & $\mathrm{~F}$ \\
\hline Soro & D1720 & 33 & $\mathrm{~F}$ \\
\hline Soro & D1721 & 30 & $\mathrm{~F}$ \\
\hline Soro & D1722 & 36 & $\mathrm{~F}$ \\
\hline Soro & D1723 & 45 & $\mathrm{~F}$ \\
\hline Soro & D1724 & 26 & M \\
\hline Soro & D1725 & 77 & $\mathrm{~F}$ \\
\hline Soro & D1726 & 0 & $M$ \\
\hline Soro & D1727 & 47 & M \\
\hline Soro & D1728 & 45 & $M$ \\
\hline Soro & D1729 & 78 & $\mathrm{~F}$ \\
\hline Soro & D1730 & 61 & $M$ \\
\hline Soro & D1731 & 36 & $\mathrm{~F}$ \\
\hline Soro & D1732 & 52 & $\mathrm{~F}$ \\
\hline Soro & D1733 & 45 & M \\
\hline Soro & D1734 & 35 & $\mathrm{~F}$ \\
\hline Soro & D1735 & 79 & $\mathrm{~F}$ \\
\hline Soro & D1736 & 77 & $M$ \\
\hline Soro & D1737 & 60 & $\mathrm{~F}$ \\
\hline Soro & D1738 & 30 & $\mathrm{~F}$ \\
\hline Soro & D1739 & 0 & $\mathrm{~F}$ \\
\hline Soro & D1740 & 0 & $\mathrm{~F}$ \\
\hline Soro & D1741 & 0 & $M$ \\
\hline Soro & D1742 & 55 & $\mathrm{~F}$ \\
\hline Soro & D1743 & 15 & $\mathrm{~F}$ \\
\hline Soro & D1744 & 0 & $\mathrm{~F}$ \\
\hline Soro & D1745 & 41 & $\mathrm{~F}$ \\
\hline Soro & D1746 & 68 & $\mathrm{~F}$ \\
\hline Soro & D1747 & 61 & $M$ \\
\hline Soro & D1748 & 59 & $\mathrm{~F}$ \\
\hline
\end{tabular}




\begin{tabular}{|c|c|c|c|}
\hline Soro & D1749 & 0 & $\mathrm{~F}$ \\
\hline Soro & D1750 & 41 & $M$ \\
\hline Soro & D1751 & 29 & M \\
\hline Soro & D1752 & 48 & $\mathrm{~F}$ \\
\hline Soro & D1753 & 51 & $\mathrm{~F}$ \\
\hline Soro & D1754 & 65 & $M$ \\
\hline Soro & D1755 & 29 & M \\
\hline Liquor/LCR & D1756 & 60 & $\mathrm{~F}$ \\
\hline Soro & D1757 & 53 & M \\
\hline Soro & D1758 & 77 & $M$ \\
\hline Soro & D1759 & 44 & $\mathrm{~F}$ \\
\hline Soro & D1760 & 44 & $\mathrm{~F}$ \\
\hline Soro & D1761 & 87 & $\mathrm{~F}$ \\
\hline Soro & D1762 & 69 & $\mathrm{M}$ \\
\hline Soro & D1763 & 56 & $\mathrm{~F}$ \\
\hline Soro & D1764 & 74 & M \\
\hline Soro & D1765 & 81 & $\mathrm{~F}$ \\
\hline Soro & D1766 & 60 & M \\
\hline Soro & D1767 & 0 & M \\
\hline Soro & D1768 & 0 & M \\
\hline Soro & D1769 & 80 & $\mathrm{~F}$ \\
\hline Soro & D1770 & 92 & M \\
\hline Soro & D1771 & 78 & M \\
\hline Soro & D1772 & 38 & M \\
\hline Soro & D1773 & 49 & $\mathrm{~F}$ \\
\hline Soro & D1774 & 80 & $\mathrm{~F}$ \\
\hline Soro & D1775 & 67 & $\mathrm{~F}$ \\
\hline Soro & D1776 & 60 & M \\
\hline Soro & D1777 & 63 & $\mathrm{~F}$ \\
\hline Soro & D1778 & 20 & M \\
\hline Soro & D1779 & 80 & M \\
\hline Soro & D1780 & 41 & $\mathrm{~F}$ \\
\hline Soro & D1781 & 64 & $\mathrm{~F}$ \\
\hline Soro & D1782 & 49 & M \\
\hline Soro & D1783 & 59 & M \\
\hline Soro & D1784 & 56 & $\mathrm{~F}$ \\
\hline Soro & D1785 & 59 & $\mathrm{~F}$ \\
\hline Soro & D1786 & 57 & $\mathrm{~F}$ \\
\hline Soro & D1787 & 60 & $M$ \\
\hline Soro & D1788 & 67 & M \\
\hline Soro & D1789 & 59 & M \\
\hline Soro & D1790 & 53 & $\mathrm{~F}$ \\
\hline Soro & D1791 & 51 & $\mathrm{~F}$ \\
\hline Soro & D1792 & 60 & $\mathrm{~F}$ \\
\hline Soro & D1793 & 65 & $\mathrm{~F}$ \\
\hline Soro & D1794 & 60 & $\mathrm{~F}$ \\
\hline Soro & D1795 & 90 & $\mathrm{~F}$ \\
\hline Soro & D1796 & 72 & $\mathrm{~F}$ \\
\hline Soro & D1797 & 22 & M \\
\hline Soro & D1798 & 51 & $\mathrm{~F}$ \\
\hline
\end{tabular}




\begin{tabular}{|c|c|c|c|}
\hline Soro & D1799 & 43 & $\mathrm{~F}$ \\
\hline Soro & D1800 & 49 & $\mathrm{~F}$ \\
\hline Soro & D1801 & 85 & $\mathrm{~F}$ \\
\hline Soro & D1802 & 0 & $\mathrm{~F}$ \\
\hline Soro & D1803 & 31 & $\mathrm{~F}$ \\
\hline Soro & D1804 & 31 & $\mathrm{~F}$ \\
\hline Soro & D1805 & 31 & $\mathrm{~F}$ \\
\hline Soro & D1806 & 60 & $\mathrm{~F}$ \\
\hline Soro & D1807 & 36 & $\mathrm{~F}$ \\
\hline Soro & D1808 & 41 & $\mathrm{~F}$ \\
\hline Soro & D1809 & 59 & $\mathrm{~F}$ \\
\hline Soro & D1810 & 89 & $\mathrm{~F}$ \\
\hline Liquor/LCR & D1811 & 0 & $M$ \\
\hline Soro & D1812 & 89 & M \\
\hline Soro & D1813 & 36 & M \\
\hline Soro & D1814 & 49 & $M$ \\
\hline Soro & D1815 & 46 & $\mathrm{~F}$ \\
\hline Soro & D1816 & 61 & $\mathrm{~F}$ \\
\hline Soro & D1817 & 73 & $\mathrm{~F}$ \\
\hline Soro & D1818 & 63 & $\mathrm{~F}$ \\
\hline Soro & D1819 & 80 & M \\
\hline Soro & D1820 & 33 & M \\
\hline Soro & D1821 & 0 & M \\
\hline Liquor/LCR & D1822 & 54 & M \\
\hline Soro & D1823 & 63 & M \\
\hline Soro & D1824 & 47 & M \\
\hline Liquor/LCR & D1825 & 50 & M \\
\hline Soro & D1826 & 82 & M \\
\hline Soro & D1827 & 39 & M \\
\hline Soro & D1828 & 41 & M \\
\hline Soro & D1829 & 72 & M \\
\hline Soro & D1830 & 56 & $M$ \\
\hline Soro & D1831 & 72 & M \\
\hline Soro & D1832 & 76 & $\mathrm{~F}$ \\
\hline Soro & D1833 & 71 & M \\
\hline Soro & D1834 & 40 & $M$ \\
\hline Soro & D1835 & 87 & $\mathrm{~F}$ \\
\hline Soro & D1836 & 68 & M \\
\hline Soro & D1837 & 64 & $M$ \\
\hline Liquor/LCR & D1838 & 62 & $\mathrm{~F}$ \\
\hline Soro & D1839 & 41 & $\mathrm{~F}$ \\
\hline Soro & D1840 & 31 & M \\
\hline Soro & D1841 & 0 & M \\
\hline Soro & D1842 & 40 & $\mathrm{M}$ \\
\hline Soro & D1843 & 38 & $\mathrm{~F}$ \\
\hline Soro & D1844 & 82 & M \\
\hline Soro & D1845 & 71 & M \\
\hline Soro & D1846 & 43 & $\mathrm{~F}$ \\
\hline Liquor/LCR & D1847 & 84 & $M$ \\
\hline Soro & D1848 & 26 & $\mathrm{~F}$ \\
\hline
\end{tabular}




\begin{tabular}{|c|c|c|c|}
\hline Soro & D1849 & 9 & $M$ \\
\hline Soro & D1850 & 73 & $\mathrm{~F}$ \\
\hline Soro & D1851 & 83 & $\mathrm{~F}$ \\
\hline Soro & D1852 & 59 & $\mathrm{~F}$ \\
\hline Soro & D1853 & 26 & M \\
\hline Liquor/LCR & D1854 & 29 & $\mathrm{~F}$ \\
\hline Soro & D1855 & 87 & M \\
\hline Soro & D1856 & 83 & $\mathrm{~F}$ \\
\hline Liquor/LCR & D1857 & 27 & $\mathrm{~F}$ \\
\hline Soro & D1858 & 19 & $M$ \\
\hline Soro & D1859 & 42 & $\mathrm{~F}$ \\
\hline Soro & D1860 & 21 & $\mathrm{~F}$ \\
\hline Soro & D1861 & 46 & $\mathrm{~F}$ \\
\hline Soro & D1862 & 87 & M \\
\hline Soro & D1863 & 81 & M \\
\hline Soro & D1864 & 57 & M \\
\hline Soro & D1865 & 48 & $M$ \\
\hline Soro & D1866 & 91 & M \\
\hline Soro & D1867 & 34 & M \\
\hline Soro & D1868 & 0 & $\mathrm{~F}$ \\
\hline Soro & D1869 & 0 & $\mathrm{~F}$ \\
\hline Soro & D1870 & 0 & M \\
\hline Liquor/LCR & D1871 & 43 & $\mathrm{~F}$ \\
\hline Soro & D1872 & 50 & M \\
\hline Soro & D1873 & 78 & $\mathrm{~F}$ \\
\hline Soro & D1874 & 40 & $\mathrm{~F}$ \\
\hline Soro & D1875 & 77 & M \\
\hline Soro & D1876 & 52 & $\mathrm{~F}$ \\
\hline Soro & D1877 & 11 & M \\
\hline Soro & D1878 & 0 & $\mathrm{~F}$ \\
\hline Soro & D1879 & 13 & M \\
\hline Soro & D1880 & 0 & $\mathrm{~F}$ \\
\hline Soro & D1881 & 32 & M \\
\hline Soro & D1882 & 52 & M \\
\hline Liquor/LCR & D1883 & 66 & M \\
\hline Soro & D1884 & 66 & $\mathrm{~F}$ \\
\hline Soro & D1885 & 45 & M \\
\hline Soro & D1886 & 86 & M \\
\hline Soro & D1887 & 75 & $M$ \\
\hline Soro & D1888 & 23 & $\mathrm{~F}$ \\
\hline Soro & D1889 & 34 & M \\
\hline Soro & D1890 & 21 & M \\
\hline Soro & D1891 & 34 & M \\
\hline Soro & D1892 & 35 & M \\
\hline Soro & D1893 & 34 & M \\
\hline Soro & D1894 & 84 & M \\
\hline Soro & D1895 & 70 & M \\
\hline Soro & D1896 & 92 & M \\
\hline Soro & D1897 & 62 & $\mathrm{~F}$ \\
\hline Soro & D1898 & 94 & $\mathrm{~F}$ \\
\hline
\end{tabular}




\begin{tabular}{|c|c|c|c|}
\hline Soro & D1899 & 33 & $\mathrm{~F}$ \\
\hline Soro & D1900 & 33 & $\mathrm{~F}$ \\
\hline Soro & D1901 & 82 & M \\
\hline Soro & D1902 & 77 & $\mathrm{~F}$ \\
\hline Soro & D1903 & 0 & $\mathrm{~F}$ \\
\hline Soro & D1904 & 9 & $M$ \\
\hline Soro & D1905 & 56 & M \\
\hline Soro & D1906 & 59 & M \\
\hline Soro & D1907 & 49 & M \\
\hline Soro & D1908 & 69 & $M$ \\
\hline Soro & D1909 & 69 & M \\
\hline Soro & D1910 & 24 & M \\
\hline Soro & D1911 & 36 & M \\
\hline Soro & D1912 & 0 & $\mathrm{~F}$ \\
\hline Soro & D1913 & 0 & $\mathrm{~F}$ \\
\hline Soro & D1914 & 0 & $\mathrm{~F}$ \\
\hline Soro & D1915 & 71 & $M$ \\
\hline Soro & D1916 & 64 & $\mathrm{~F}$ \\
\hline Soro & D1917 & 63 & M \\
\hline Soro & D1918 & 36 & $\mathrm{~F}$ \\
\hline Soro & D1919 & 63 & $\mathrm{~F}$ \\
\hline Soro & D1920 & 68 & M \\
\hline Soro & D1921 & 38 & M \\
\hline Soro & D1922 & 58 & $\mathrm{~F}$ \\
\hline Soro & D1923 & 0 & M \\
\hline Soro & D1924 & 68 & $\mathrm{~F}$ \\
\hline Soro & D1925 & 87 & M \\
\hline Soro & D1926 & 64 & $\mathrm{~F}$ \\
\hline Soro & D1927 & 68 & $\mathrm{~F}$ \\
\hline Soro & D1928 & 67 & 1 \\
\hline Soro & D1929 & 62 & M \\
\hline Soro & D1930 & 85 & $M$ \\
\hline Soro & D1931 & 71 & M \\
\hline Soro & D1932 & 38 & M \\
\hline Soro & D1933 & 43 & M \\
\hline Soro & D1934 & 59 & $\mathrm{~F}$ \\
\hline Soro & D1935 & 79 & $\mathrm{M}$ \\
\hline Soro & D1936 & 49 & $\mathrm{~F}$ \\
\hline Soro & D1937 & 68 & M \\
\hline Soro & D1938 & 32 & M \\
\hline Soro & D1939 & 53 & $\mathrm{~F}$ \\
\hline Soro & D1940 & 11 & M \\
\hline Soro & D1941 & 77 & $\mathrm{~F}$ \\
\hline Soro & D1942 & 69 & $\mathrm{~F}$ \\
\hline Soro & D1943 & 72 & $\mathrm{~F}$ \\
\hline Liquor/LCR & D1944 & 77 & $M$ \\
\hline Soro & D1945 & 44 & $\mathrm{~F}$ \\
\hline Soro & D1946 & 0 & M \\
\hline Soro & D1947 & 49 & $\mathrm{~F}$ \\
\hline Soro & D1948 & 73 & M \\
\hline
\end{tabular}




\begin{tabular}{|c|c|c|c|}
\hline Soro & D1949 & 82 & $\mathrm{~F}$ \\
\hline Soro & D1950 & 39 & $M$ \\
\hline Soro & D1951 & 96 & M \\
\hline Soro & D1952 & 53 & $\mathrm{~F}$ \\
\hline Soro & D1953 & 0 & $\mathrm{~F}$ \\
\hline Soro & D1954 & 84 & $M$ \\
\hline Soro & D1955 & 92 & M \\
\hline Soro & D1956 & 11 & M \\
\hline Soro & D1957 & 56 & $\mathrm{~F}$ \\
\hline Soro & D1958 & 92 & $M$ \\
\hline Soro & D1959 & 73 & $\mathrm{~F}$ \\
\hline Soro & D1960 & 52 & M \\
\hline Soro & D1961 & 61 & $\mathrm{~F}$ \\
\hline Soro & D1962 & 59 & $\mathrm{~F}$ \\
\hline Soro & D1963 & 82 & M \\
\hline Soro & D1964 & 42 & $M$ \\
\hline Soro & D1965 & 64 & $M$ \\
\hline Soro & D1966 & 51 & M \\
\hline Soro & D1967 & 64 & M \\
\hline Soro & D1968 & 0 & $\mathrm{~F}$ \\
\hline Soro & D1969 & 59 & $\mathrm{~F}$ \\
\hline Soro & D1970 & 11 & M \\
\hline Liquor/LCR & D1971 & 76 & $\mathrm{~F}$ \\
\hline Soro & D1972 & 56 & M \\
\hline Soro & D1973 & 41 & $\mathrm{~F}$ \\
\hline Soro & D1974 & 63 & M \\
\hline Soro & D1975 & 78 & M \\
\hline Soro & D1976 & 72 & $\mathrm{~F}$ \\
\hline Soro & D1977 & 88 & M \\
\hline Soro & D1978 & 64 & $\mathrm{~F}$ \\
\hline Soro & D1979 & 19 & M \\
\hline Soro & D1980 & 49 & $\mathrm{~F}$ \\
\hline Soro & D1981 & 31 & $\mathrm{~F}$ \\
\hline Soro & D1982 & 70 & $\mathrm{~F}$ \\
\hline Soro & D1983 & 47 & M \\
\hline Soro & D1984 & 31 & $\mathrm{~F}$ \\
\hline Soro & D1985 & 55 & M \\
\hline Soro & D1986 & 43 & $\mathrm{~F}$ \\
\hline Soro & D1987 & 44 & $\mathrm{~F}$ \\
\hline Soro & D1988 & 51 & $\mathrm{~F}$ \\
\hline Soro & D1989 & 69 & M \\
\hline Soro & D1990 & 57 & $\mathrm{~F}$ \\
\hline Soro & D1991 & 39 & $\mathrm{~F}$ \\
\hline Soro & D1992 & 20 & $\mathrm{~F}$ \\
\hline Soro & D1993 & 49 & M \\
\hline Soro & D1994 & 35 & $\mathrm{~F}$ \\
\hline Soro & D1995 & 6 & M \\
\hline Soro & D1996 & 63 & M \\
\hline Soro & D1997 & 63 & M \\
\hline Soro & D1998 & 42 & $\mathrm{~F}$ \\
\hline
\end{tabular}




\begin{tabular}{|c|c|c|c|}
\hline Soro & D1999 & 54 & $\mathrm{~F}$ \\
\hline Soro & D2000 & 79 & $\mathrm{~F}$ \\
\hline Soro & D2001 & 27 & M \\
\hline Soro & D2002 & 23 & M \\
\hline Soro & D2003 & 14 & M \\
\hline Soro & D2004 & 0 & M \\
\hline Soro & D2005 & 64 & M \\
\hline Soro & D2006 & 89 & M \\
\hline Soro & D2007 & 64 & M \\
\hline Soro & D2008 & 59 & $\mathrm{~F}$ \\
\hline Soro & D2009 & 0 & $\mathrm{~F}$ \\
\hline Soro & D2010 & 29 & M \\
\hline Soro & D2011 & 70 & $\mathrm{~F}$ \\
\hline Soro & D2012 & 48 & $\mathrm{M}$ \\
\hline Soro & D2013 & 53 & M \\
\hline Liquor/LCR & D2014 & 85 & $M$ \\
\hline Soro & D2015 & 40 & $M$ \\
\hline Soro & D2016 & 37 & $\mathrm{~F}$ \\
\hline Soro & D2017 & 84 & $\mathrm{~F}$ \\
\hline Soro & D2018 & 42 & $\mathrm{~F}$ \\
\hline Soro & D2019 & 56 & M \\
\hline Soro & D2020 & 40 & $\mathrm{~F}$ \\
\hline Soro & D2021 & 0 & M \\
\hline Soro & D2022 & 51 & $\mathrm{~F}$ \\
\hline Soro & D2023 & 99 & $\mathrm{~F}$ \\
\hline Soro & D2024 & 65 & $\mathrm{~F}$ \\
\hline Soro & D2025 & 57 & $\mathrm{~F}$ \\
\hline Soro & D2026 & 87 & M \\
\hline Soro & D2027 & 52 & $\mathrm{~F}$ \\
\hline Soro & D2028 & 62 & $\mathrm{M}$ \\
\hline Soro & D2029 & 62 & $\mathrm{~F}$ \\
\hline Soro & D2030 & 41 & $\mathrm{~F}$ \\
\hline Soro & D2031 & 82 & $\mathrm{~F}$ \\
\hline Soro & D2032 & 43 & $\mathrm{~F}$ \\
\hline Soro & D2033 & 49 & $\mathrm{~F}$ \\
\hline Soro & D2034 & 67 & $\mathrm{M}$ \\
\hline Soro & D2035 & 69 & $\mathrm{~F}$ \\
\hline Soro & D2036 & 71 & $\mathrm{~F}$ \\
\hline Soro & D2037 & 43 & $\mathrm{~F}$ \\
\hline Soro & D2038 & 35 & M \\
\hline Soro & D2039 & 18 & $\mathrm{~F}$ \\
\hline Soro & D2040 & 95 & M \\
\hline Soro & D2041 & 27 & $\mathrm{~F}$ \\
\hline Soro & D2042 & 0 & $\mathrm{M}$ \\
\hline Liquor/LCR & D2043 & 40 & M \\
\hline Liquor/LCR & D2044 & 77 & M \\
\hline Soro & D2045 & 45 & $\mathrm{~F}$ \\
\hline Soro & D2046 & 58 & M \\
\hline Soro & D2047 & 33 & M \\
\hline Soro & D2048 & 21 & $\mathrm{~F}$ \\
\hline
\end{tabular}




\begin{tabular}{|c|c|c|c|}
\hline Soro & D2049 & 61 & $M$ \\
\hline Soro & D2050 & 80 & $M$ \\
\hline Soro & D2051 & 79 & $\mathrm{~F}$ \\
\hline Soro & D2052 & 77 & M \\
\hline Soro & D2053 & 43 & $\mathrm{~F}$ \\
\hline Soro & D2054 & 54 & $M$ \\
\hline Liquor/LCR & D2055 & 46 & M \\
\hline Soro & D2056 & 65 & M \\
\hline Liquor/LCR & D2057 & 79 & $\mathrm{~F}$ \\
\hline Soro & D2058 & 81 & $M$ \\
\hline Soro & D2059 & 71 & $\mathrm{~F}$ \\
\hline Soro & D2060 & 0 & M \\
\hline Soro & D2061 & 68 & M \\
\hline Soro & D2062 & 52 & $\mathrm{M}$ \\
\hline Soro & D2063 & 68 & M \\
\hline Soro & D2064 & 51 & $\mathrm{~F}$ \\
\hline Soro & D2065 & 51 & $M$ \\
\hline Soro & D2066 & 69 & M \\
\hline Soro & D2067 & 19 & M \\
\hline Soro & D2068 & 51 & M \\
\hline Soro & D2069 & 93 & M \\
\hline Soro & D2070 & 40 & $\mathrm{M}$ \\
\hline Soro & D2071 & 55 & $\mathrm{~F}$ \\
\hline Soro & D2072 & 66 & $\mathrm{~F}$ \\
\hline Soro & D2073 & 28 & M \\
\hline Soro & D2074 & 93 & $\mathrm{~F}$ \\
\hline Soro & D2075 & 49 & M \\
\hline Soro & D2076 & 76 & M \\
\hline Soro & D2077 & 0 & $\mathrm{M}$ \\
\hline Soro & D2078 & 43 & $\mathrm{~F}$ \\
\hline Soro & D2079 & 8 & M \\
\hline Soro & D2080 & 85 & $\mathrm{~F}$ \\
\hline Soro & D2081 & 74 & M \\
\hline Soro & D2082 & 66 & M \\
\hline Soro & D2083 & 66 & M \\
\hline Liquor/LCR & D2084 & 44 & $\mathrm{~F}$ \\
\hline Soro & D2085 & 37 & $\mathrm{~F}$ \\
\hline Soro & D2086 & 80 & $\mathrm{~F}$ \\
\hline Soro & D2087 & 62 & $M$ \\
\hline Soro & D2088 & 61 & $\mathrm{~F}$ \\
\hline Soro & D2089 & 61 & M \\
\hline Soro & D2090 & 33 & M \\
\hline Liquor/LCR & D2091 & 36 & $\mathrm{~F}$ \\
\hline Soro & D2092 & 60 & $\mathrm{~F}$ \\
\hline Soro & D2093 & 0 & M \\
\hline Soro & D2094 & 37 & M \\
\hline Soro & D2095 & 60 & $\mathrm{~F}$ \\
\hline Soro & D2096 & 36 & $\mathrm{~F}$ \\
\hline Soro & D2097 & 47 & $\mathrm{~F}$ \\
\hline Soro & D2098 & 51 & $\mathrm{~F}$ \\
\hline
\end{tabular}




\begin{tabular}{|c|c|c|c|}
\hline Soro & D2099 & 60 & $M$ \\
\hline Soro & D2100 & 19 & $M$ \\
\hline Soro & D2101 & 66 & $\mathrm{~F}$ \\
\hline Soro & D2102 & 0 & $\mathrm{~F}$ \\
\hline Liquor/LCR & D2103 & 82 & $\mathrm{~F}$ \\
\hline Soro & D2104 & 71 & $M$ \\
\hline Soro & D2105 & 68 & M \\
\hline Soro & D2106 & 85 & M \\
\hline Soro & D2107 & 45 & $\mathrm{~F}$ \\
\hline Soro & D2108 & 47 & $\mathrm{~F}$ \\
\hline Soro & D2109 & 47 & $\mathrm{~F}$ \\
\hline Soro & D2110 & 56 & M \\
\hline Soro & D2111 & 58 & $\mathrm{~F}$ \\
\hline Soro & D2112 & 61 & M \\
\hline Soro & D2113 & 50 & $\mathrm{~F}$ \\
\hline Soro & D2114 & 75 & M \\
\hline Soro & D2115 & 41 & $M$ \\
\hline Soro & D2116 & 76 & $\mathrm{~F}$ \\
\hline Soro & D2117 & 59 & $\mathrm{~F}$ \\
\hline Soro & D2118 & 50 & M \\
\hline Soro & D2119 & 60 & $\mathrm{~F}$ \\
\hline Soro & D2120 & 74 & M \\
\hline Liquor/LCR & D2121 & 11 & M \\
\hline Soro & D2122 & 54 & $\mathrm{~F}$ \\
\hline Soro & D2123 & 39 & $\mathrm{~F}$ \\
\hline Liquor/LCR & D2124 & 50 & M \\
\hline Liquor/LCR & D2125 & 50 & M \\
\hline Soro & D2126 & 51 & M \\
\hline Soro & D2127 & 71 & $\mathrm{~F}$ \\
\hline Soro & D2128 & 79 & M \\
\hline Soro & D2129 & 0 & M \\
\hline Soro & D2130 & 39 & $M$ \\
\hline Soro & D2131 & 79 & M \\
\hline Soro & D2132 & 0 & M \\
\hline Soro & D2133 & 32 & M \\
\hline Soro & D2134 & 76 & $\mathrm{~F}$ \\
\hline Soro & D2135 & 44 & $\mathrm{~F}$ \\
\hline Soro & D2136 & 81 & M \\
\hline Soro & D2137 & 41 & $M$ \\
\hline Soro & D2138 & 41 & $\mathrm{~F}$ \\
\hline Soro & D2139 & 86 & M \\
\hline Soro & D2140 & 39 & M \\
\hline Soro & D2141 & 53 & M \\
\hline Soro & D2142 & 40 & $\mathrm{~F}$ \\
\hline Soro & D2143 & 29 & M \\
\hline Soro & D2144 & 49 & $\mathrm{~F}$ \\
\hline Soro & D2145 & 60 & M \\
\hline Soro & D2146 & 49 & $\mathrm{~F}$ \\
\hline Soro & D2147 & 44 & $M$ \\
\hline Soro & D2148 & 71 & M \\
\hline
\end{tabular}




\begin{tabular}{|c|c|c|c|}
\hline Soro & D2149 & 81 & $\mathrm{~F}$ \\
\hline Soro & D2150 & 95 & $\mathrm{~F}$ \\
\hline Soro & D2151 & 68 & M \\
\hline Soro & D2152 & 94 & M \\
\hline Soro & D2153 & 33 & $\mathrm{~F}$ \\
\hline Soro & D2154 & 81 & $\mathrm{~F}$ \\
\hline Soro & D2155 & 83 & $\mathrm{~F}$ \\
\hline Soro & D2156 & 7 & $\mathrm{~F}$ \\
\hline Soro & D2157 & 82 & $\mathrm{~F}$ \\
\hline Soro & D2158 & 22 & $M$ \\
\hline Soro & D2159 & 50 & M \\
\hline Soro & D2160 & 85 & M \\
\hline Soro & D2161 & 38 & M \\
\hline Soro & D2162 & 71 & $\mathrm{M}$ \\
\hline Soro & D2163 & 54 & M \\
\hline Soro & D2164 & 0 & $\mathrm{~F}$ \\
\hline Soro & D2165 & 0 & $\mathrm{~F}$ \\
\hline Soro & D2166 & 47 & M \\
\hline Soro & D2167 & 58 & $\mathrm{~F}$ \\
\hline Soro & D2168 & 39 & M \\
\hline Soro & D2169 & 83 & M \\
\hline Soro & D2170 & 69 & M \\
\hline Soro & D2171 & 47 & M \\
\hline Soro & D2172 & 36 & $\mathrm{~F}$ \\
\hline Soro & D2173 & 87 & $\mathrm{~F}$ \\
\hline Soro & D2174 & 85 & $\mathrm{~F}$ \\
\hline Soro & D2175 & 56 & M \\
\hline Soro & D2176 & 3 & M \\
\hline Soro & D2177 & 42 & $\mathrm{~F}$ \\
\hline Soro & D2178 & 53 & $\mathrm{~F}$ \\
\hline Soro & D2179 & 80 & M \\
\hline Soro & D2180 & 52 & $\mathrm{~F}$ \\
\hline Soro & D2181 & 0 & M \\
\hline Soro & D2182 & 69 & M \\
\hline Soro & D2183 & 60 & M \\
\hline Liquor/LCR & D2184 & 80 & $\mathrm{~F}$ \\
\hline Soro & D2185 & 48 & $\mathrm{~F}$ \\
\hline Soro & D2186 & 59 & M \\
\hline Soro & D2187 & 61 & $M$ \\
\hline Soro & D2188 & 77 & M \\
\hline Soro & D2189 & 34 & $\mathrm{~F}$ \\
\hline Soro & D2190 & 31 & M \\
\hline Soro & D2191 & 62 & $\mathrm{~F}$ \\
\hline Soro & D2192 & 66 & $\mathrm{M}$ \\
\hline Soro & D2193 & 69 & $\mathrm{~F}$ \\
\hline Soro & D2194 & 70 & $M$ \\
\hline Soro & D2195 & 66 & $\mathrm{~F}$ \\
\hline Soro & D2196 & 20 & M \\
\hline Soro & D2197 & 90 & M \\
\hline Soro & D2198 & 31 & M \\
\hline
\end{tabular}




\begin{tabular}{|c|c|c|c|}
\hline Soro & D2199 & 48 & $M$ \\
\hline Soro & D2200 & 82 & $\mathrm{~F}$ \\
\hline Soro & D2201 & 80 & M \\
\hline Soro & D2202 & 80 & M \\
\hline Soro & D2203 & 68 & $\mathrm{~F}$ \\
\hline Soro & D2204 & 67 & $\mathrm{~F}$ \\
\hline Soro & D2205 & 93 & $\mathrm{~F}$ \\
\hline Soro & D2206 & 0 & M \\
\hline Soro & D2207 & 0 & M \\
\hline Soro & D2208 & 71 & $\mathrm{~F}$ \\
\hline Soro & D2209 & 34 & M \\
\hline Soro & D2210 & 45 & $\mathrm{~F}$ \\
\hline Soro & D2211 & 48 & M \\
\hline Soro & D2212 & 64 & M \\
\hline Soro & D2213 & 56 & $\mathrm{~F}$ \\
\hline Soro & D2214 & 39 & $\mathrm{~F}$ \\
\hline Liquor/LCR & D2215 & 40 & $\mathrm{~F}$ \\
\hline Soro & D2216 & 44 & $\mathrm{~F}$ \\
\hline Soro & D2217 & 70 & M \\
\hline Liquor/LCR & D2218 & 44 & $\mathrm{~F}$ \\
\hline Soro & D2219 & & M \\
\hline Liquor/LCR & D2220 & & M \\
\hline Soro & D2221 & 77 & M \\
\hline Soro & D2222 & 61 & M \\
\hline Soro & D2223 & 85 & $\mathrm{~F}$ \\
\hline Liquor/LCR & D2224 & 42 & $\mathrm{~F}$ \\
\hline Soro & D2225 & 39 & $\mathrm{~F}$ \\
\hline Soro & D2226 & 44 & M \\
\hline Soro & D2227 & 39 & $\mathrm{~F}$ \\
\hline Liquor/LCR & D2228 & 77 & $\mathrm{M}$ \\
\hline Soro & D2229 & & $\mathrm{F}$ \\
\hline Soro & D2230 & 73 & M \\
\hline Soro & D2231 & 76 & M \\
\hline Soro & D2232 & 69 & M \\
\hline Soro & D2233 & 67 & M \\
\hline Soro & D2234 & 37 & $\mathrm{~F}$ \\
\hline Soro & D2235 & 70 & $\mathrm{~F}$ \\
\hline Soro & D2236 & 45 & $\mathrm{~F}$ \\
\hline Soro & D2237 & 47 & $\mathrm{~F}$ \\
\hline Soro & D2238 & 75 & M \\
\hline Soro & D2239 & 51 & $\mathrm{~F}$ \\
\hline Soro & D2240 & 41 & M \\
\hline Soro & D2241 & 44 & $\mathrm{~F}$ \\
\hline Soro & D2242 & & M \\
\hline Soro & D2243 & 83 & $\mathrm{~F}$ \\
\hline Liquor/LCR & D2244 & 30 & M \\
\hline Soro & D2245 & 49 & $\mathrm{~F}$ \\
\hline Soro & D2246 & 62 & $\mathrm{~F}$ \\
\hline Soro & D2247 & 85 & M \\
\hline Liquor/LCR & D2248 & 74 & $\mathrm{~F}$ \\
\hline
\end{tabular}




\begin{tabular}{|c|c|c|c|}
\hline Soro & D2249 & 53 & 1 \\
\hline Soro & D2250 & & $\mathrm{F}$ \\
\hline Soro & D2251 & 33 & $\mathrm{~F}$ \\
\hline Soro & D2252 & 70 & $M$ \\
\hline Soro & D2253 & 35 & $\mathrm{~F}$ \\
\hline Soro & D2254 & 60 & $\mathrm{~F}$ \\
\hline Soro & D2255 & 82 & $\mathrm{~F}$ \\
\hline Liquor/LCR & D2256 & 62 & $M$ \\
\hline Soro & D2257 & & $M$ \\
\hline Soro & D2258 & 50 & $\mathrm{~F}$ \\
\hline Soro & D2259 & 74 & $M$ \\
\hline Soro & D2260 & 32 & $\mathrm{~F}$ \\
\hline Soro & D2261 & 22 & $M$ \\
\hline Soro & D2262 & 36 & $M$ \\
\hline Soro & D2263 & 39 & $M$ \\
\hline Soro & D2264 & 40 & $M$ \\
\hline Soro & D2265 & 48 & $M$ \\
\hline Soro & D2266 & 64 & $M$ \\
\hline Soro & D2267 & 81 & $\mathrm{~F}$ \\
\hline Soro & D2268 & 57 & $\mathrm{~F}$ \\
\hline Soro & D2269 & 32 & $M$ \\
\hline Soro & D2270 & 49 & $\mathrm{~F}$ \\
\hline Soro & D2271 & 33 & $M$ \\
\hline Soro & D2272 & 59 & $\mathrm{~F}$ \\
\hline Soro & D2273 & & $M$ \\
\hline Soro & D2274 & 43 & $\mathrm{~F}$ \\
\hline Soro & D2275 & 57 & $M$ \\
\hline Soro & D2276 & 57 & $M$ \\
\hline Soro & D2277 & 66 & $M$ \\
\hline Soro & D2278 & 57 & $M$ \\
\hline Soro & D2279 & 68 & $M$ \\
\hline Soro & D2280 & 48 & $\mathrm{~F}$ \\
\hline Soro & D2281 & 85 & $\mathrm{~F}$ \\
\hline Liquor/LCR & D2282 & 83 & $\mathrm{M}$ \\
\hline Soro & D2283 & 56 & $\mathrm{~F}$ \\
\hline Soro & D2284 & 55 & $M$ \\
\hline Soro & D2285 & 59 & $M$ \\
\hline Soro & D2286 & 50 & $\mathrm{~F}$ \\
\hline Soro & D2287 & 85 & $M$ \\
\hline Soro & D2288 & 39 & $\mathrm{~F}$ \\
\hline Soro & D2289 & 42 & $M$ \\
\hline Soro & D2290 & 63 & $M$ \\
\hline Soro & D2291 & 63 & $\mathrm{~F}$ \\
\hline Soro & D2292 & 25 & $\mathrm{~F}$ \\
\hline Soro & D2293 & 80 & $M$ \\
\hline Soro & D2294 & & $M$ \\
\hline Soro & D2295 & & $M$ \\
\hline Soro & D2296 & 92 & $\mathrm{~F}$ \\
\hline Soro & D2297 & 82 & $\mathrm{~F}$ \\
\hline Soro & D2298 & 80 & $F$ \\
\hline
\end{tabular}




\begin{tabular}{|llll}
\hline Soro & $\mathrm{D} 2299$ & 65 & $\mathrm{M}$ \\
\hline Soro & $\mathrm{D} 2300$ & 29 & $\mathrm{M}$ \\
\hline Soro & $\mathrm{D} 2301$ & 45 & $\mathrm{~F}$ \\
\hline Liquor/LCR & $\mathrm{D} 2302$ & 48 & $\mathrm{M}$ \\
\hline Liquor/LCR & $\mathrm{D} 2303$ & 48 & $\mathrm{M}$ \\
\hline Soro & $\mathrm{D} 2304$ & 65 & $\mathrm{~F}$ \\
\hline Soro & $\mathrm{D} 2305$ & 51 & $\mathrm{M}$ \\
\hline Soro & $\mathrm{D} 2306$ & & $\mathrm{~F}$ \\
\hline Soro & $\mathrm{D} 2307$ & 80 & $\mathrm{M}$ \\
\hline Liquor/LCR & $\mathrm{D} 2308$ & 36 & $\mathrm{~F}$ \\
\hline Soro & $\mathrm{D} 2309$ & 81 & $\mathrm{M}$ \\
\hline Soro & $\mathrm{D} 2310$ & 67 & $\mathrm{~F}$ \\
\hline Soro & $\mathrm{D} 2311$ & 46 & $\mathrm{M}$ \\
\hline Soro & $\mathrm{D} 2312$ & & $\mathrm{M}$ \\
\hline Soro & $\mathrm{D} 2313$ & 62 & $\mathrm{M}$ \\
\hline Soro & $\mathrm{D} 2314$ & 63 & $\mathrm{M}$ \\
\hline Soro & $\mathrm{D} 2315$ & 47 & $\mathrm{~F}$ \\
\hline Soro & $\mathrm{D} 2316$ & 46 & $\mathrm{M}$ \\
\hline Soro & $\mathrm{D} 2317$ & 27 & $\mathrm{M}$ \\
\hline Soro & $\mathrm{D} 2318$ & & $\mathrm{~F}$ \\
\hline Soro & $\mathrm{D} 2319$ & & $\mathrm{M}$ \\
\hline Soro & $\mathrm{D} 2320$ & 43 & $\mathrm{~F}$ \\
\hline Soro & $\mathrm{D} 2321$ & 87 & $\mathrm{M}$ \\
\hline & & & \\
\hline
\end{tabular}




\begin{tabular}{|c|}
\hline Result \\
\hline Negativo \\
\hline Negativo \\
\hline Negativo \\
\hline Negativo \\
\hline Negativo \\
\hline Negativo \\
\hline Negativo \\
\hline Negativo \\
\hline Positivo \\
\hline Negativo \\
\hline Negativo \\
\hline Negativo \\
\hline Negativo \\
\hline Negativo \\
\hline Negativo \\
\hline Negativo \\
\hline Negativo \\
\hline Negativo \\
\hline Negativo \\
\hline Negativo \\
\hline Negativo \\
\hline Positivo \\
\hline Negativo \\
\hline Negativo \\
\hline Negativo \\
\hline Negativo \\
\hline Negativo \\
\hline Negativo \\
\hline Negativo \\
\hline Positivo \\
\hline Negativo \\
\hline Negativo \\
\hline Negativo \\
\hline Negativo \\
\hline Negativo \\
\hline Negativo \\
\hline Negativo \\
\hline Negativo \\
\hline Negativo \\
\hline Negativo \\
\hline Negativo \\
\hline Negativo \\
\hline Negativo \\
\hline Negativo \\
\hline Negativo \\
\hline Negativo \\
\hline Negativo \\
\hline Negativo \\
\hline
\end{tabular}




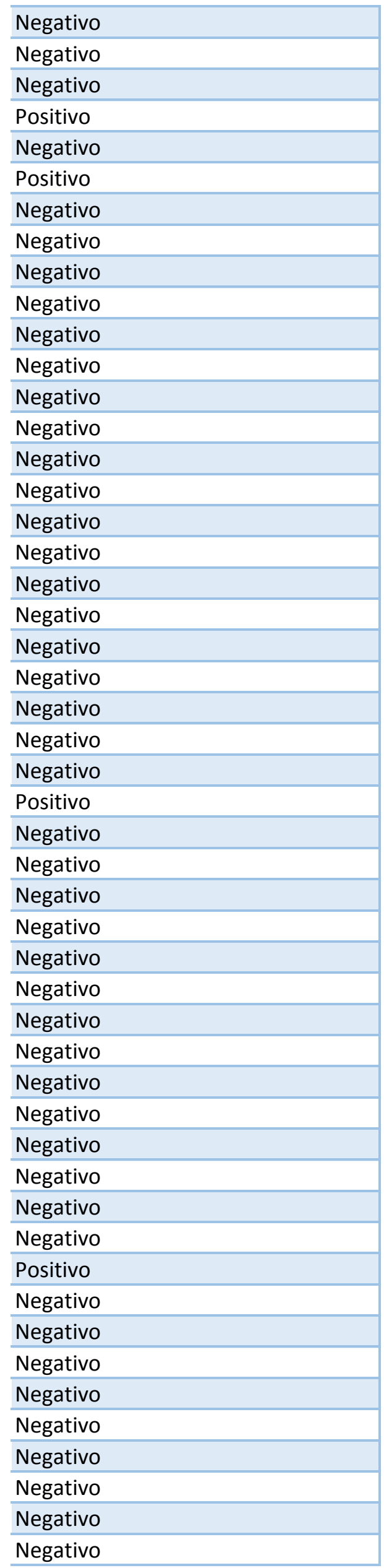




\begin{tabular}{|l|}
\hline Negativo \\
\hline Negativo \\
\hline Negativo \\
\hline Negativo \\
\hline Negativo \\
\hline Negativo \\
\hline Negativo \\
\hline Negativo \\
\hline Negativo \\
\hline Negativo \\
\hline Negativo \\
\hline Positivo \\
\hline Negativo \\
\hline Negativo \\
\hline Negativo \\
\hline Negativo \\
\hline Positivo \\
\hline Negativo \\
\hline Negativo \\
\hline Negativo \\
\hline Negativo \\
\hline Negativo \\
\hline Negativo \\
\hline Negativo \\
\hline Negativo \\
\hline Negativo \\
\hline Negativo \\
\hline Negativo \\
\hline Nesitivo \\
\hline Negativo \\
\hline Negativo \\
\hline Negativo \\
\hline Negativo \\
\hline Negativo \\
\hline Negativo \\
\hline Negativo \\
\hline Negativotivo \\
\hline Negativo \\
\hline Negativo \\
\hline Negativo \\
\hline
\end{tabular}




\begin{tabular}{|l|}
\hline Negativo \\
\hline Negativo \\
\hline Negativo \\
\hline Negativo \\
\hline Negativo \\
\hline Negativo \\
\hline Negativo \\
\hline Negativo \\
\hline Negativo \\
\hline Negativo \\
\hline Negativo \\
\hline Negativo \\
\hline Negativo \\
\hline Negativo \\
\hline Negativo \\
\hline Negativo \\
\hline Negativo \\
\hline Negativo \\
\hline Negativo \\
\hline Negativo \\
\hline Negativo \\
\hline Negativo \\
\hline Positivo \\
\hline Negativo \\
\hline Negitivo \\
\hline Negativo \\
\hline Negativo \\
\hline Negativo \\
\hline Negativativo \\
\hline Positivo \\
\hline Negativo \\
\hline Negativo \\
\hline Negativo \\
\hline Negativo \\
\hline Nesitivo \\
\hline Negativo \\
\hline Negativo \\
\hline Negativo \\
\hline Negativo \\
\hline Negativo \\
\hline
\end{tabular}




\begin{tabular}{|l|}
\hline Negativo \\
\hline Negativo \\
\hline Negativo \\
\hline Negativo \\
\hline Negativo \\
\hline Negativo \\
\hline Negativo \\
\hline Positivo \\
\hline Positivo \\
\hline Negativo \\
\hline Negativo \\
\hline Negativo \\
\hline Negativo \\
\hline Negativo \\
\hline Negativo \\
\hline Negativo \\
\hline Negativo \\
\hline Negativo \\
\hline Negativo \\
\hline Negativo \\
\hline Negativo \\
\hline Negativo \\
\hline Negativo \\
\hline Negativo \\
\hline Negativo \\
\hline Negativo \\
\hline Negativo \\
\hline Negativo \\
\hline Negativo \\
\hline Negativo \\
\hline Positivo \\
\hline Negativo \\
\hline Negativo \\
\hline Positivo \\
\hline Negativo \\
\hline Negativo \\
\hline Negativo \\
\hline Negativo \\
\hline Negativo \\
\hline Negativo \\
\hline
\end{tabular}




\begin{tabular}{|l|}
\hline Positivo \\
\hline Negativo \\
\hline Negativo \\
\hline Negativo \\
\hline Negativo \\
\hline Negativo \\
\hline Negativo \\
\hline Negativo \\
\hline Negativo \\
\hline Negativo \\
\hline Negativo \\
\hline Positivo \\
\hline Positivo \\
\hline Negativo \\
\hline Negativo \\
\hline Negativo \\
\hline Negativo \\
\hline Negativo \\
\hline Negativo \\
\hline Negativo \\
\hline Negativo \\
\hline Negativo \\
\hline Negativo \\
\hline Negativo \\
\hline Positivo \\
\hline Negativo \\
\hline Negativo \\
\hline Negativo \\
\hline Positivo \\
\hline Negativo \\
\hline Negativo \\
\hline Negativo \\
\hline Negativo \\
\hline Negativo \\
\hline Negativo \\
\hline Pegativo \\
\hline Negitivo \\
\hline Negativo \\
\hline
\end{tabular}




\begin{tabular}{|l|}
\hline Negativo \\
\hline Positivo \\
\hline Negativo \\
\hline Negativo \\
\hline Negativo \\
\hline Negativo \\
\hline Negativo \\
\hline Negativo \\
\hline Negativo \\
\hline Negativo \\
\hline Negativo \\
\hline Negativo \\
\hline Negativo \\
\hline Negativo \\
\hline Negativo \\
\hline Negativo \\
\hline Negativo \\
\hline Negativo \\
\hline Negativo \\
\hline Negativo \\
\hline Negativo \\
\hline Negativo \\
\hline Negativo \\
\hline Negativo \\
\hline Negativo \\
\hline Negativo \\
\hline Negativo \\
\hline Negativo \\
\hline Negativo \\
\hline Negativo \\
\hline Negativo \\
\hline Negativo \\
\hline Negativo \\
\hline Negativo \\
\hline Negativo \\
\hline Negativo \\
\hline Negativo \\
\hline Negativo \\
\hline Negativo \\
\hline
\end{tabular}




\begin{tabular}{|l|}
\hline Negativo \\
\hline Negativo \\
\hline Negativo \\
\hline Negativo \\
\hline Negativo \\
\hline Negativo \\
\hline Negativo \\
\hline Positivo \\
\hline Negativo \\
\hline Negativo \\
\hline Negativo \\
\hline Negativo \\
\hline Negativo \\
\hline Negativo \\
\hline Negativo \\
\hline Negativo \\
\hline Negativo \\
\hline Negativo \\
\hline Negativo \\
\hline Negativo \\
\hline Negativo \\
\hline Negativo \\
\hline Negativo \\
\hline Negativativo \\
\hline Negativo \\
\hline Negativo \\
\hline Negativo \\
\hline Positivo \\
\hline Negativo \\
\hline Negativo \\
\hline Negativo \\
\hline Negativo \\
\hline Negativo \\
\hline Negativo \\
\hline Negativo \\
\hline Negativo \\
\hline Negativo \\
\hline Negativo \\
\hline
\end{tabular}




\begin{tabular}{|c|}
\hline Negativo \\
\hline Negativo \\
\hline Negativo \\
\hline Negativo \\
\hline Positivo \\
\hline Negativo \\
\hline Negativo \\
\hline Negativo \\
\hline Negativo \\
\hline Negativo \\
\hline Positivo \\
\hline Negativo \\
\hline Negativo \\
\hline Negativo \\
\hline Negativo \\
\hline Negativo \\
\hline Negativo \\
\hline Negativo \\
\hline Negativo \\
\hline Negativo \\
\hline Negativo \\
\hline Negativo \\
\hline Negativo \\
\hline Negativo \\
\hline Negativo \\
\hline Negativo \\
\hline Negativo \\
\hline Negativo \\
\hline Negativo \\
\hline Negativo \\
\hline Negativo \\
\hline Negativo \\
\hline Negativo \\
\hline Negativo \\
\hline Negativo \\
\hline Negativo \\
\hline Positivo \\
\hline Negativo \\
\hline Negativo \\
\hline Negativo \\
\hline Negativo \\
\hline Negativo \\
\hline Negativo \\
\hline Negativo \\
\hline Negativo \\
\hline Negativo \\
\hline Negativo \\
\hline Negativo \\
\hline Negativo \\
\hline Negativo \\
\hline
\end{tabular}




\begin{tabular}{|c|}
\hline Negativo \\
\hline Negativo \\
\hline Negativo \\
\hline Negativo \\
\hline Negativo \\
\hline Negativo \\
\hline Negativo \\
\hline Negativo \\
\hline Negativo \\
\hline Negativo \\
\hline Negativo \\
\hline Negativo \\
\hline Negativo \\
\hline Negativo \\
\hline Negativo \\
\hline Negativo \\
\hline Negativo \\
\hline Negativo \\
\hline Negativo \\
\hline Negativo \\
\hline Negativo \\
\hline Negativo \\
\hline Negativo \\
\hline Negativo \\
\hline Negativo \\
\hline Negativo \\
\hline Negativo \\
\hline Negativo \\
\hline Negativo \\
\hline Positivo \\
\hline Negativo \\
\hline Negativo \\
\hline Negativo \\
\hline Negativo \\
\hline Negativo \\
\hline Negativo \\
\hline Negativo \\
\hline Negativo \\
\hline Positivo \\
\hline Negativo \\
\hline Negativo \\
\hline Negativo \\
\hline Negativo \\
\hline Negativo \\
\hline Negativo \\
\hline Positivo \\
\hline Negativo \\
\hline Negativo \\
\hline Negativo \\
\hline Negativo \\
\hline
\end{tabular}




\begin{tabular}{|c|}
\hline Negativo \\
\hline Negativo \\
\hline Positivo \\
\hline Negativo \\
\hline Negativo \\
\hline Negativo \\
\hline Negativo \\
\hline Negativo \\
\hline Negativo \\
\hline Negativo \\
\hline Negativo \\
\hline Negativo \\
\hline Negativo \\
\hline Negativo \\
\hline Negativo \\
\hline Negativo \\
\hline Negativo \\
\hline Negativo \\
\hline Negativo \\
\hline Negativo \\
\hline Negativo \\
\hline Negativo \\
\hline Negativo \\
\hline Positivo \\
\hline Negativo \\
\hline Negativo \\
\hline Negativo \\
\hline Negativo \\
\hline Positivo \\
\hline Negativo \\
\hline Negativo \\
\hline Negativo \\
\hline Negativo \\
\hline Negativo \\
\hline Negativo \\
\hline Negativo \\
\hline Negativo \\
\hline Negativo \\
\hline Negativo \\
\hline Negativo \\
\hline Negativo \\
\hline Positivo \\
\hline Positivo \\
\hline Negativo \\
\hline Positivo \\
\hline Negativo \\
\hline Negativo \\
\hline Negativo \\
\hline Negativo \\
\hline Negativo \\
\hline
\end{tabular}




\begin{tabular}{|l|}
\hline Negativo \\
\hline Positivo \\
\hline Negativo \\
\hline Negativo \\
\hline Negativo \\
\hline Negativo \\
\hline Negativo \\
\hline Negativo \\
\hline Positivo \\
\hline Negativo \\
\hline Negativo \\
\hline Negativo \\
\hline Negativo \\
\hline Negativo \\
\hline Negativo \\
\hline Negativo \\
\hline Negativo \\
\hline Negativo \\
\hline Negativo \\
\hline Negativo \\
\hline Negativo \\
\hline Negativo \\
\hline Negativo \\
\hline Negativo \\
\hline Negativo \\
\hline Negativo \\
\hline Negativo \\
\hline Negativo \\
\hline Negativo \\
\hline Negativo \\
\hline Negativo \\
\hline Negativo \\
\hline Positivo \\
\hline Negativo \\
\hline Negativo \\
\hline Negativo \\
\hline Negativo \\
\hline Negativo \\
\hline Negativo \\
\hline Negativo \\
\hline
\end{tabular}




\begin{tabular}{|l|}
\hline Positivo \\
\hline Negativo \\
\hline Negativo \\
\hline Negativo \\
\hline Negativo \\
\hline Negativo \\
\hline Negativo \\
\hline Negativo \\
\hline Negativo \\
\hline Negativo \\
\hline Negativo \\
\hline Negativo \\
\hline Negativo \\
\hline Negativo \\
\hline Positivo \\
\hline Negativo \\
\hline Negativo \\
\hline Negativo \\
\hline Negativo \\
\hline Negativo \\
\hline Negativo \\
\hline Negativo \\
\hline Negativo \\
\hline Negativo \\
\hline Negativativo \\
\hline Negativo \\
\hline Negativo \\
\hline Negativo \\
\hline Negativo \\
\hline Negativo \\
\hline Negativo \\
\hline Negativo \\
\hline Negativo \\
\hline Negativo \\
\hline Negativo \\
\hline Negativotivo \\
\hline Negativo \\
\hline Negativo \\
\hline
\end{tabular}




\begin{tabular}{|l|}
\hline Negativo \\
\hline Negativo \\
\hline Positivo \\
\hline Negativo \\
\hline Negativo \\
\hline Negativo \\
\hline Negativo \\
\hline Negativo \\
\hline Negativo \\
\hline Negativo \\
\hline Negativo \\
\hline Negativo \\
\hline Negativo \\
\hline Negativo \\
\hline Negativo \\
\hline Negativo \\
\hline Negativo \\
\hline Negativo \\
\hline Negativo \\
\hline Negativo \\
\hline Negativo \\
\hline Negativo \\
\hline Negativo \\
\hline Negativo \\
\hline Negativo \\
\hline Negativo \\
\hline Negativo \\
\hline Negativo \\
\hline Negativo \\
\hline Negativo \\
\hline Negativo \\
\hline Negativo \\
\hline Negativo \\
\hline Negativo \\
\hline Negativo \\
\hline Negativo \\
\hline Negativo \\
\hline Negativo \\
\hline
\end{tabular}




\begin{tabular}{|l|}
\hline Negativo \\
\hline Negativo \\
\hline Negativo \\
\hline Negativo \\
\hline Negativo \\
\hline Negativo \\
\hline Positivo \\
\hline Negativo \\
\hline Negativo \\
\hline Negativo \\
\hline Negativo \\
\hline Negativo \\
\hline Negativo \\
\hline Negativo \\
\hline Negativo \\
\hline Negativo \\
\hline Positivo \\
\hline Negativo \\
\hline Negativo \\
\hline Negativo \\
\hline Negativo \\
\hline Negativo \\
\hline Negativo \\
\hline Negativo \\
\hline Negativo \\
\hline Negativo \\
\hline Negativo \\
\hline Negativo \\
\hline Negativo \\
\hline Negativo \\
\hline Negativo \\
\hline Negativo \\
\hline Negativo \\
\hline Negativo \\
\hline Negativo \\
\hline Negativo \\
\hline Negativotivo \\
\hline Negativo \\
\hline Negativo \\
\hline Negativo \\
\hline
\end{tabular}




\begin{tabular}{|c|}
\hline Negativo \\
\hline Negativo \\
\hline Positivo \\
\hline Negativo \\
\hline Negativo \\
\hline Negativo \\
\hline Negativo \\
\hline Negativo \\
\hline Negativo \\
\hline Negativo \\
\hline Negativo \\
\hline Positivo \\
\hline Negativo \\
\hline Negativo \\
\hline Negativo \\
\hline Negativo \\
\hline Negativo \\
\hline Negativo \\
\hline Negativo \\
\hline Negativo \\
\hline Negativo \\
\hline Negativo \\
\hline Negativo \\
\hline Negativo \\
\hline Negativo \\
\hline Negativo \\
\hline Negativo \\
\hline Negativo \\
\hline Negativo \\
\hline Negativo \\
\hline Negativo \\
\hline Negativo \\
\hline Negativo \\
\hline Negativo \\
\hline Negativo \\
\hline Positivo \\
\hline Negativo \\
\hline Negativo \\
\hline Negativo \\
\hline Negativo \\
\hline Negativo \\
\hline Negativo \\
\hline Negativo \\
\hline Positivo \\
\hline Negativo \\
\hline Negativo \\
\hline Positivo \\
\hline Negativo \\
\hline Negativo \\
\hline Negativo \\
\hline
\end{tabular}




\begin{tabular}{|c|}
\hline Negativo \\
\hline Negativo \\
\hline Negativo \\
\hline Negativo \\
\hline Negativo \\
\hline Negativo \\
\hline Negativo \\
\hline Negativo \\
\hline Negativo \\
\hline Positivo \\
\hline Negativo \\
\hline Negativo \\
\hline Positivo \\
\hline Negativo \\
\hline Negativo \\
\hline Negativo \\
\hline Negativo \\
\hline Negativo \\
\hline Negativo \\
\hline Negativo \\
\hline Negativo \\
\hline Negativo \\
\hline Negativo \\
\hline Negativo \\
\hline Negativo \\
\hline Negativo \\
\hline Negativo \\
\hline Negativo \\
\hline Negativo \\
\hline Negativo \\
\hline Negativo \\
\hline Negativo \\
\hline Negativo \\
\hline Negativo \\
\hline Negativo \\
\hline Negativo \\
\hline Negativo \\
\hline Negativo \\
\hline Negativo \\
\hline Negativo \\
\hline Negativo \\
\hline Negativo \\
\hline Negativo \\
\hline Negativo \\
\hline Positivo \\
\hline Negativo \\
\hline Negativo \\
\hline Negativo \\
\hline Negativo \\
\hline Negativo \\
\hline
\end{tabular}




\begin{tabular}{|c|}
\hline Negativo \\
\hline Negativo \\
\hline Negativo \\
\hline Positivo \\
\hline Negativo \\
\hline Negativo \\
\hline Negativo \\
\hline Negativo \\
\hline Negativo \\
\hline Negativo \\
\hline Negativo \\
\hline Negativo \\
\hline Negativo \\
\hline Negativo \\
\hline Negativo \\
\hline Negativo \\
\hline Negativo \\
\hline Negativo \\
\hline Negativo \\
\hline Negativo \\
\hline Negativo \\
\hline Positivo \\
\hline Negativo \\
\hline Negativo \\
\hline Negativo \\
\hline Negativo \\
\hline Negativo \\
\hline Negativo \\
\hline Positivo \\
\hline Negativo \\
\hline Negativo \\
\hline Negativo \\
\hline Negativo \\
\hline Negativo \\
\hline Negativo \\
\hline Negativo \\
\hline Negativo \\
\hline Negativo \\
\hline Negativo \\
\hline Negativo \\
\hline Negativo \\
\hline Negativo \\
\hline Negativo \\
\hline Negativo \\
\hline Negativo \\
\hline Negativo \\
\hline Negativo \\
\hline Negativo \\
\hline Negativo \\
\hline Negativo \\
\hline
\end{tabular}




\begin{tabular}{|c|}
\hline Negativo \\
\hline Negativo \\
\hline Negativo \\
\hline Negativo \\
\hline Negativo \\
\hline Negativo \\
\hline Negativo \\
\hline Negativo \\
\hline Negativo \\
\hline Negativo \\
\hline Negativo \\
\hline Negativo \\
\hline Negativo \\
\hline Positivo \\
\hline Negativo \\
\hline Negativo \\
\hline Negativo \\
\hline Negativo \\
\hline Negativo \\
\hline Negativo \\
\hline Negativo \\
\hline Negativo \\
\hline Negativo \\
\hline Negativo \\
\hline Negativo \\
\hline Negativo \\
\hline Negativo \\
\hline Negativo \\
\hline Negativo \\
\hline Negativo \\
\hline Negativo \\
\hline Negativo \\
\hline Negativo \\
\hline Negativo \\
\hline Negativo \\
\hline Negativo \\
\hline Negativo \\
\hline Negativo \\
\hline Negativo \\
\hline Negativo \\
\hline Negativo \\
\hline Negativo \\
\hline Negativo \\
\hline Negativo \\
\hline Negativo \\
\hline Negativo \\
\hline Negativo \\
\hline Negativo \\
\hline Negativo \\
\hline Negativo \\
\hline
\end{tabular}




\begin{tabular}{|l|}
\hline Negativo \\
\hline Negativo \\
\hline Negativo \\
\hline Negativo \\
\hline Negativo \\
\hline Negativo \\
\hline Negativo \\
\hline Negativo \\
\hline Negativo \\
\hline Negativo \\
\hline Negativo \\
\hline Negativo \\
\hline Negativo \\
\hline Negativo \\
\hline Negativo \\
\hline Negativo \\
\hline Negativo \\
\hline Negativo \\
\hline Negativo \\
\hline Negativo \\
\hline Negativo \\
\hline Negativo \\
\hline Negativo \\
\hline Negativo \\
\hline Negativo \\
\hline Negativo \\
\hline Negativo \\
\hline Negativo \\
\hline Negativo \\
\hline Negativo \\
\hline Negativo \\
\hline Negativo \\
\hline Negativo \\
\hline Negativo \\
\hline Negativo \\
\hline Negativo \\
\hline Negativo \\
\hline Negativo \\
\hline Negativo \\
\hline
\end{tabular}




\begin{tabular}{|l|}
\hline Negativo \\
\hline Negativo \\
\hline Negativo \\
\hline Negativo \\
\hline Negativo \\
\hline Positivo \\
\hline Negativo \\
\hline Negativo \\
\hline Negativo \\
\hline Negativo \\
\hline Negativo \\
\hline Negativo \\
\hline Negativo \\
\hline Negativo \\
\hline Negativo \\
\hline Negativo \\
\hline Negativo \\
\hline Negativo \\
\hline Negativo \\
\hline Negativo \\
\hline Negativo \\
\hline Negativo \\
\hline Negativo \\
\hline Negativo \\
\hline Negativo \\
\hline Negativo \\
\hline Negativativo \\
\hline Negativo \\
\hline Negativo \\
\hline Negativo \\
\hline Negativo \\
\hline Negativo \\
\hline Negativo \\
\hline Negativo \\
\hline Negativo \\
\hline Negativo \\
\hline Negativo \\
\hline Negativo \\
\hline Negativo \\
\hline
\end{tabular}




\begin{tabular}{|l|}
\hline Negativo \\
\hline Positivo \\
\hline Negativo \\
\hline Negativo \\
\hline Positivo \\
\hline Negativo \\
\hline Negativo \\
\hline Negativo \\
\hline Negativo \\
\hline Negativo \\
\hline Negativo \\
\hline Positivo \\
\hline Negativo \\
\hline Negativo \\
\hline Negativo \\
\hline Negativo \\
\hline Negativo \\
\hline Negativo \\
\hline Negativo \\
\hline Negativo \\
\hline Negativo \\
\hline Positivo \\
\hline Positivo \\
\hline Negativo \\
\hline Negativo \\
\hline Negativo \\
\hline Negativo \\
\hline Negativo \\
\hline Negativo \\
\hline Negativo \\
\hline Negativo \\
\hline Positivo \\
\hline Positivo \\
\hline Negativo \\
\hline Negativo \\
\hline Negativo \\
\hline Negativo \\
\hline Negativo \\
\hline Negativo \\
\hline Negativo \\
\hline
\end{tabular}




\begin{tabular}{|l|}
\hline Negativo \\
\hline Negativo \\
\hline Negativo \\
\hline Negativo \\
\hline Negativo \\
\hline Negativo \\
\hline Positivo \\
\hline Positivo \\
\hline Positivo \\
\hline Negativo \\
\hline Negativo \\
\hline Negativo \\
\hline Negativo \\
\hline Negativo \\
\hline Negativo \\
\hline Negativo \\
\hline Negativo \\
\hline Negativo \\
\hline Negativo \\
\hline Negativo \\
\hline Positivo \\
\hline Negativo \\
\hline Negativo \\
\hline Negativo \\
\hline Negativo \\
\hline Negativo \\
\hline Negativo \\
\hline Negativo \\
\hline Negativo \\
\hline Negativo \\
\hline Negativo \\
\hline Negativo \\
\hline Negativo \\
\hline Negativo \\
\hline Negativo \\
\hline Negativo \\
\hline Negativotivo \\
\hline Negativo \\
\hline Negativo \\
\hline
\end{tabular}




\begin{tabular}{|l|}
\hline Negativo \\
\hline Positivo \\
\hline Negativo \\
\hline Negativo \\
\hline Negativo \\
\hline Negativo \\
\hline Positivo \\
\hline Negativo \\
\hline Negativo \\
\hline Negativo \\
\hline Negativo \\
\hline Negativo \\
\hline Negativo \\
\hline Negativo \\
\hline Negativo \\
\hline Negativo (ver observações) \\
\hline Negativo \\
\hline Negativo \\
\hline Negativo \\
\hline Negativo \\
\hline Negativo \\
\hline Negativo \\
\hline Negativo \\
\hline Negativo \\
\hline Negativo \\
\hline Negativo \\
\hline Negativo \\
\hline Negativo \\
\hline Negativo \\
\hline Negativo \\
\hline Negativo \\
\hline Negativo \\
\hline Negativo \\
\hline Negativo \\
\hline Positivo \\
\hline Nesitivo \\
\hline Negativativo \\
\hline Negativo \\
\hline Negativo \\
\hline
\end{tabular}




\begin{tabular}{|l|}
\hline Negativo \\
\hline Negativo \\
\hline Negativo \\
\hline Negativo \\
\hline Negativo \\
\hline Negativo \\
\hline Negativo \\
\hline Negativo \\
\hline Negativo \\
\hline Negativo \\
\hline Negativo \\
\hline Negativo \\
\hline Positivo \\
\hline Negativo \\
\hline Negativo \\
\hline Negativo \\
\hline Negativo \\
\hline Negativo \\
\hline Negativo \\
\hline Negativo \\
\hline Negativo \\
\hline Nositivo \\
\hline Negativativo \\
\hline Negitivo \\
\hline Negativo \\
\hline Negativo \\
\hline Positivo \\
\hline Negativo \\
\hline Negativo \\
\hline Negativo \\
\hline Negativo \\
\hline Positivo \\
\hline Nesitivo \\
\hline Negativo \\
\hline Negativo \\
\hline Negativo \\
\hline Negativo \\
\hline \\
\hline Negativo \\
\hline
\end{tabular}




\begin{tabular}{|l|}
\hline Negativo \\
\hline Negativo \\
\hline Positivo \\
\hline Negativo \\
\hline Negativo \\
\hline Negativo \\
\hline Negativo \\
\hline Negativo \\
\hline Negativo \\
\hline Positivo \\
\hline Negativo \\
\hline Negativo \\
\hline Negativo \\
\hline Negativo \\
\hline Negativo \\
\hline Negativo \\
\hline Negativo \\
\hline Negativo \\
\hline Negativo \\
\hline Negativo \\
\hline Negativo \\
\hline Negativo \\
\hline Negativo \\
\hline Negativo \\
\hline Negativo \\
\hline Negativo \\
\hline Negativo \\
\hline Negativo \\
\hline Negativo \\
\hline Negativo \\
\hline Positivo \\
\hline Negativo \\
\hline Negativo \\
\hline Negativo \\
\hline Negativo \\
\hline Negativo \\
\hline Negativo \\
\hline Negativo \\
\hline Negativo \\
\hline Negativo \\
\hline
\end{tabular}




\begin{tabular}{|l|}
\hline Negativo \\
\hline Negativo \\
\hline Negativo \\
\hline Negativo \\
\hline Negativo \\
\hline Negativo \\
\hline Negativo \\
\hline Positivo \\
\hline Negativo \\
\hline Negativo \\
\hline Negativo \\
\hline Negativo \\
\hline Negativo \\
\hline Negativo \\
\hline Negativo \\
\hline Negativo \\
\hline Negativo \\
\hline Negativo \\
\hline Negativo \\
\hline Positivo \\
\hline Negativo \\
\hline Negativo \\
\hline Positivo \\
\hline Negativo \\
\hline Negativo \\
\hline Negativo \\
\hline Negativo \\
\hline Negativo \\
\hline Negativo \\
\hline Negativo \\
\hline Negativo \\
\hline Negativo \\
\hline Negativo \\
\hline Negativo \\
\hline Negativo \\
\hline Negativo \\
\hline Negativo \\
\hline Negativo \\
\hline Negativo \\
\hline Negativo \\
\hline
\end{tabular}




\begin{tabular}{|l|}
\hline Negativo \\
\hline Negativo \\
\hline Positivo \\
\hline Negativo \\
\hline Negativo \\
\hline Negativo \\
\hline Negativo \\
\hline Negativo \\
\hline Negativo \\
\hline Negativo \\
\hline Negativo \\
\hline Negativo \\
\hline Negativo \\
\hline Negativo \\
\hline Negativo \\
\hline Negativo \\
\hline Negativo \\
\hline Negativo \\
\hline Negativo \\
\hline Negativo \\
\hline Negativo \\
\hline Negativo \\
\hline Negativo \\
\hline Negativo \\
\hline Negativo \\
\hline Negativo \\
\hline Negativo \\
\hline Negativo \\
\hline Negativo \\
\hline Negativo \\
\hline Negativo \\
\hline Negativo \\
\hline Negativo \\
\hline Negativo \\
\hline Negativo \\
\hline Negativo \\
\hline Negativo \\
\hline Negativo \\
\hline
\end{tabular}




\begin{tabular}{|l|}
\hline Negativo \\
\hline Positivo \\
\hline Negativo \\
\hline Negativo \\
\hline Negativo \\
\hline Negativo \\
\hline Negativo \\
\hline Negativo \\
\hline Negativo \\
\hline Negativo \\
\hline Negativo \\
\hline Negativo \\
\hline Negativo \\
\hline Negativo \\
\hline Negativo \\
\hline Negativo \\
\hline Negativo \\
\hline Negativo \\
\hline Negativo \\
\hline Negitivo \\
\hline Negativo \\
\hline Negativo \\
\hline Negativo \\
\hline Negativo \\
\hline Negativo \\
\hline Negativo \\
\hline Negativo \\
\hline Negativo \\
\hline Negativo \\
\hline Negativo \\
\hline Negativo \\
\hline Negativo \\
\hline Negativo \\
\hline Negativo \\
\hline Negativo \\
\hline Negativo \\
\hline Negativo \\
\hline
\end{tabular}




\begin{tabular}{|c|}
\hline Negativo \\
\hline Negativo \\
\hline Negativo \\
\hline Negativo \\
\hline Negativo \\
\hline Negativo \\
\hline Negativo \\
\hline Negativo \\
\hline Negativo \\
\hline Negativo \\
\hline Negativo \\
\hline Negativo \\
\hline Negativo \\
\hline Negativo \\
\hline Negativo \\
\hline Negativo \\
\hline Negativo \\
\hline Negativo \\
\hline Negativo \\
\hline Negativo \\
\hline Negativo \\
\hline Negativo \\
\hline Negativo \\
\hline Negativo \\
\hline Negativo \\
\hline Negativo \\
\hline Negativo \\
\hline Positivo \\
\hline Negativo \\
\hline Negativo \\
\hline Negativo \\
\hline Negativo \\
\hline Negativo \\
\hline Negativo \\
\hline Negativo \\
\hline Negativo \\
\hline Negativo \\
\hline Negativo \\
\hline Negativo \\
\hline Negativo \\
\hline Negativo \\
\hline Negativo \\
\hline Negativo \\
\hline Negativo \\
\hline Positivo \\
\hline Negativo \\
\hline Negativo \\
\hline Negativo \\
\hline Negativo \\
\hline Negativo \\
\hline
\end{tabular}




\begin{tabular}{|c|}
\hline Negativo \\
\hline Negativo \\
\hline Negativo \\
\hline Negativo \\
\hline Negativo \\
\hline Negativo \\
\hline Negativo \\
\hline Negativo \\
\hline Negativo \\
\hline Negativo \\
\hline Negativo \\
\hline Negativo \\
\hline Negativo \\
\hline Negativo \\
\hline Negativo \\
\hline Negativo \\
\hline Negativo \\
\hline Negativo \\
\hline Positivo \\
\hline Negativo \\
\hline Negativo \\
\hline Negativo \\
\hline Negativo \\
\hline Negativo \\
\hline Positivo \\
\hline Negativo \\
\hline Negativo \\
\hline Negativo \\
\hline Negativo \\
\hline Negativo \\
\hline Negativo \\
\hline Negativo \\
\hline Negativo \\
\hline Negativo \\
\hline Negativo \\
\hline Negativo \\
\hline Negativo \\
\hline Positivo \\
\hline Negativo \\
\hline Negativo \\
\hline Negativo \\
\hline Negativo \\
\hline Negativo \\
\hline Negativo \\
\hline Negativo \\
\hline Negativo \\
\hline Negativo \\
\hline Negativo \\
\hline Negativo \\
\hline Negativo \\
\hline
\end{tabular}




\begin{tabular}{|l|}
\hline Negativo \\
\hline Negativo \\
\hline Negativo \\
\hline Negativo \\
\hline Negativo \\
\hline Negativo \\
\hline Negativo \\
\hline Negativo \\
\hline Negativo \\
\hline Positivo \\
\hline Negativo \\
\hline Negativo \\
\hline Negativo \\
\hline Positivo \\
\hline Positivo \\
\hline Negativo \\
\hline Negativo \\
\hline Negativo \\
\hline Negativo \\
\hline Negativo \\
\hline Negativo \\
\hline Positivo \\
\hline Negativo \\
\hline Negativo \\
\hline Negativo \\
\hline Negativo \\
\hline Negativo \\
\hline Negativo \\
\hline Negativo \\
\hline Negativo \\
\hline Negativo \\
\hline Negativo \\
\hline Positivo \\
\hline Negativo \\
\hline Negativo \\
\hline Negativo \\
\hline Negativo \\
\hline Negativo \\
\hline Negativo \\
\hline Negativo \\
\hline
\end{tabular}




\begin{tabular}{|l|}
\hline Negativo \\
\hline Negativo \\
\hline Negativo \\
\hline Negativo \\
\hline Negativo \\
\hline Negativo \\
\hline Negativo \\
\hline Negativo \\
\hline Negativo \\
\hline Negativo \\
\hline Negativo \\
\hline Negativo \\
\hline Negativo \\
\hline Negativo \\
\hline Positivo \\
\hline Negativo \\
\hline Negativo \\
\hline Negativo \\
\hline Negativo \\
\hline Negativo \\
\hline Negativo \\
\hline Negativo \\
\hline Negativo \\
\hline Negativo \\
\hline Negativo \\
\hline Negativo \\
\hline Negativo \\
\hline Negativo \\
\hline Negativo \\
\hline Negativo \\
\hline Negativo \\
\hline Negativo \\
\hline Negativo \\
\hline Negativo \\
\hline Negativo \\
\hline Negativo \\
\hline Negativo \\
\hline Negativo \\
\hline Negativo \\
\hline
\end{tabular}




\begin{tabular}{|c|}
\hline Negativo \\
\hline Negativo \\
\hline Negativo \\
\hline Negativo \\
\hline Negativo \\
\hline Negativo \\
\hline Negativo \\
\hline Negativo \\
\hline Negativo \\
\hline Negativo \\
\hline Negativo \\
\hline Negativo \\
\hline Positivo \\
\hline Negativo \\
\hline Negativo \\
\hline Negativo \\
\hline Negativo \\
\hline Negativo \\
\hline Negativo \\
\hline Negativo \\
\hline Negativo \\
\hline Negativo \\
\hline Negativo \\
\hline Negativo \\
\hline Negativo \\
\hline Negativo \\
\hline Negativo \\
\hline Negativo \\
\hline Negativo \\
\hline Negativo \\
\hline Negativo \\
\hline Negativo \\
\hline Negativo \\
\hline Negativo \\
\hline Negativo \\
\hline Negativo \\
\hline Negativo \\
\hline Negativo \\
\hline Negativo \\
\hline Negativo \\
\hline Positivo \\
\hline Negativo \\
\hline Negativo \\
\hline Negativo \\
\hline Positivo \\
\hline Negativo \\
\hline Negativo \\
\hline Negativo \\
\hline Negativo \\
\hline Negativo \\
\hline
\end{tabular}




\begin{tabular}{|l|}
\hline Negativo \\
\hline Negativo \\
\hline Negativo \\
\hline Negativo \\
\hline Negativo \\
\hline Negativo \\
\hline Negativo \\
\hline Negativo \\
\hline Negativo \\
\hline Negativo \\
\hline Negativo \\
\hline Negativo \\
\hline Negativo \\
\hline Negativo \\
\hline Negativo \\
\hline Negativo \\
\hline Negativo \\
\hline Negativo \\
\hline Negativo \\
\hline Negativo \\
\hline Negativo \\
\hline Negativo \\
\hline Negativo \\
\hline Negativo \\
\hline Negativo \\
\hline Negativo \\
\hline Negativo \\
\hline Negativo \\
\hline Negativo \\
\hline Negativo \\
\hline Negativo \\
\hline Negativo \\
\hline Negativo \\
\hline Negativo \\
\hline Negativo \\
\hline Negativo \\
\hline Negativo \\
\hline Negativo \\
\hline Negativo \\
\hline
\end{tabular}




\begin{tabular}{|c|}
\hline Negativo \\
\hline Negativo \\
\hline Positivo \\
\hline Negativo \\
\hline Negativo \\
\hline Negativo \\
\hline Positivo \\
\hline Negativo \\
\hline Negativo \\
\hline Negativo \\
\hline Negativo \\
\hline Negativo \\
\hline Negativo \\
\hline Negativo \\
\hline Negativo \\
\hline Negativo \\
\hline Negativo \\
\hline Negativo \\
\hline Negativo \\
\hline Negativo \\
\hline Negativo \\
\hline Negativo \\
\hline Negativo \\
\hline Negativo \\
\hline Negativo \\
\hline Negativo \\
\hline Negativo \\
\hline Negativo \\
\hline Negativo \\
\hline Negativo \\
\hline Negativo \\
\hline Negativo \\
\hline Negativo \\
\hline Negativo \\
\hline Negativo \\
\hline Negativo \\
\hline Negativo \\
\hline Negativo \\
\hline Negativo \\
\hline Negativo \\
\hline Negativo \\
\hline Negativo \\
\hline Negativo \\
\hline Negativo \\
\hline Negativo \\
\hline Negativo \\
\hline Negativo \\
\hline Negativo \\
\hline Negativo \\
\hline Negativo \\
\hline
\end{tabular}




\begin{tabular}{|l|}
\hline Negativo \\
\hline Negativo \\
\hline Negativo \\
\hline Negativo \\
\hline Negativo \\
\hline Negativo \\
\hline Negativo \\
\hline Negativo \\
\hline Negativo \\
\hline Negativo \\
\hline Negativo \\
\hline Negativo \\
\hline Negativo \\
\hline Negativo \\
\hline Negativo \\
\hline Negativo \\
\hline Negativo \\
\hline Negativo \\
\hline Positivo \\
\hline Negativo \\
\hline Negativo \\
\hline Negativo \\
\hline Negativo \\
\hline Negativo \\
\hline Negativo \\
\hline Negativo \\
\hline Negativo \\
\hline Negativo \\
\hline Negativo \\
\hline Negativo \\
\hline Negativo \\
\hline Negativo \\
\hline Negativo \\
\hline Negativo \\
\hline Negativo \\
\hline Negativo \\
\hline Negativo \\
\hline Negativo \\
\hline Negativo \\
\hline
\end{tabular}




\begin{tabular}{|l|}
\hline Negativo \\
\hline Negativo \\
\hline Negativo \\
\hline Negativo \\
\hline Negativo \\
\hline Negativo \\
\hline Negativo \\
\hline Negativo \\
\hline Negativo \\
\hline Positivo \\
\hline Negativo \\
\hline Negativo \\
\hline Negativo \\
\hline Negativo \\
\hline Negativo \\
\hline Negativo \\
\hline Negativo \\
\hline Negativo \\
\hline Negativo \\
\hline Negativo \\
\hline Negativo \\
\hline Negativo \\
\hline Negativo \\
\hline Negativo \\
\hline Negativo \\
\hline Negativo \\
\hline Negativo \\
\hline Negativo \\
\hline Negativo \\
\hline Negativo \\
\hline Positivo \\
\hline Negativo \\
\hline Negativo \\
\hline Negativo \\
\hline Negativo \\
\hline Negativo \\
\hline Negativo \\
\hline Negativo \\
\hline
\end{tabular}




\begin{tabular}{|l|}
\hline Negativo \\
\hline Negativo \\
\hline Negativo \\
\hline Negativo \\
\hline Negativo \\
\hline Negativo \\
\hline Negativo \\
\hline Negativo \\
\hline Negativo \\
\hline Negativo \\
\hline Negativo \\
\hline Negativo \\
\hline Negativo \\
\hline Negativo \\
\hline Negativo \\
\hline Negativo \\
\hline Negativo \\
\hline Negativo \\
\hline Negativo \\
\hline Negativo \\
\hline Negativo \\
\hline Negativo \\
\hline Positivo \\
\hline Negitivo \\
\hline Negativo \\
\hline Negativo \\
\hline Negativativo \\
\hline Negativo \\
\hline Negativo \\
\hline Negativo \\
\hline Negativo \\
\hline Negativo \\
\hline Negativo \\
\hline Negativo \\
\hline Negativo \\
\hline Negativo \\
\hline Negativo \\
\hline Negativo \\
\hline
\end{tabular}




\begin{tabular}{|l|}
\hline Negativo \\
\hline Negativo \\
\hline Negativo \\
\hline Negativo \\
\hline Negativo \\
\hline Negativo \\
\hline Negativo \\
\hline Negativo \\
\hline Negativo \\
\hline Negativo \\
\hline Positivo \\
\hline Negativo \\
\hline Negativo \\
\hline Negativo \\
\hline Negativo \\
\hline Negativo \\
\hline Negativo \\
\hline Negativo \\
\hline Negativo \\
\hline Negativo \\
\hline Negativo \\
\hline Positivo \\
\hline Negativo \\
\hline Negativo \\
\hline Negativo \\
\hline Negativo \\
\hline Negativo \\
\hline Negativo \\
\hline Negativo \\
\hline Negativo \\
\hline Negativo \\
\hline Negativo \\
\hline Negativo \\
\hline Nesitivo \\
\hline Negativo \\
\hline Negativo \\
\hline Negativo \\
\hline \\
\hline Negativo \\
\hline
\end{tabular}




\begin{tabular}{|l|}
\hline Negativo \\
\hline Negativo \\
\hline Negativo \\
\hline Negativo \\
\hline Negativo \\
\hline Negativo \\
\hline Negativo \\
\hline Negativo \\
\hline Negativo \\
\hline Negativo \\
\hline Negativo \\
\hline Negativo \\
\hline Negativo \\
\hline Negativo \\
\hline Negativo \\
\hline Negativo \\
\hline Positivo \\
\hline Negativo \\
\hline Negativo \\
\hline Negativo \\
\hline Negativo \\
\hline Negativo \\
\hline Negativo \\
\hline Negativo \\
\hline Negativo \\
\hline Negativo \\
\hline Negativo \\
\hline Negativo \\
\hline Negativo \\
\hline Negativo \\
\hline Negativo \\
\hline Negativo \\
\hline Negativo \\
\hline Negativo \\
\hline Negativo \\
\hline Negativo \\
\hline Negativo \\
\hline Negativo \\
\hline
\end{tabular}




\begin{tabular}{|l|}
\hline Negativo \\
\hline Negativo \\
\hline Negativo \\
\hline Negativo \\
\hline Negativo \\
\hline Negativo \\
\hline Negativo \\
\hline Negativo \\
\hline Negativo \\
\hline Negativo \\
\hline Negativo \\
\hline Positivo \\
\hline Negativo \\
\hline Negativo \\
\hline Negativo \\
\hline Negativo \\
\hline Negativo \\
\hline Negativo \\
\hline Positivo \\
\hline Negativo \\
\hline Negativo \\
\hline Negativo \\
\hline Negativo \\
\hline Negativo \\
\hline Negativo \\
\hline Negativo \\
\hline Negativo \\
\hline Negativo \\
\hline Negativo \\
\hline Positivo \\
\hline Negativo \\
\hline Negativo \\
\hline Negativo \\
\hline Negativo \\
\hline Positivo \\
\hline Negativo \\
\hline Negativotivo \\
\hline Negativo \\
\hline Negativo \\
\hline Negativo \\
\hline
\end{tabular}




\begin{tabular}{|l|}
\hline Positivo \\
\hline Negativo \\
\hline Negativo \\
\hline Negativo \\
\hline Negativo \\
\hline Negativo \\
\hline Negativo \\
\hline Negativo \\
\hline Negativo \\
\hline Negativo \\
\hline Negativo \\
\hline Negativo \\
\hline Negativo \\
\hline Negativo \\
\hline Negativo \\
\hline Negativo \\
\hline Negativo \\
\hline Positivo \\
\hline Positivo \\
\hline Negativo \\
\hline Negativo \\
\hline Positivo \\
\hline Negativo \\
\hline Negativo \\
\hline Negativo \\
\hline Positivo \\
\hline Negativo \\
\hline Negativo \\
\hline Negativo \\
\hline Negativo \\
\hline Negativo \\
\hline Negativo \\
\hline Negativo \\
\hline Negativo \\
\hline Negativo \\
\hline Negativo \\
\hline Negativativo \\
\hline Negativo \\
\hline
\end{tabular}




\begin{tabular}{|c|}
\hline Negativo \\
\hline Negativo \\
\hline Negativo \\
\hline Negativo \\
\hline Negativo \\
\hline Negativo \\
\hline Negativo \\
\hline Negativo \\
\hline Negativo \\
\hline Negativo \\
\hline Positivo \\
\hline Negativo \\
\hline Negativo \\
\hline Negativo \\
\hline Negativo \\
\hline Negativo \\
\hline Negativo \\
\hline Positivo \\
\hline Negativo \\
\hline Negativo \\
\hline Negativo \\
\hline Positivo \\
\hline Positivo \\
\hline Negativo \\
\hline Negativo \\
\hline Negativo \\
\hline Negativo \\
\hline Negativo \\
\hline Negativo \\
\hline Negativo \\
\hline Positivo \\
\hline Negativo \\
\hline Negativo \\
\hline Positivo \\
\hline Negativo \\
\hline Negativo \\
\hline Negativo \\
\hline Negativo \\
\hline Negativo \\
\hline Positivo \\
\hline Negativo \\
\hline Negativo \\
\hline Positivo \\
\hline Negativo \\
\hline Negativo \\
\hline Negativo \\
\hline Negativo \\
\hline Negativo \\
\hline Negativo \\
\hline Negativo \\
\hline
\end{tabular}




\begin{tabular}{|l|}
\hline Negativo \\
\hline Negativo \\
\hline Negativo \\
\hline Negativo \\
\hline Negativo \\
\hline Negativo \\
\hline Negativo \\
\hline Positivo \\
\hline Positivo \\
\hline Negativo \\
\hline Negativo \\
\hline Negativo \\
\hline Negativo \\
\hline Negativo \\
\hline Negativo \\
\hline Positivo \\
\hline Negativo \\
\hline Negativo \\
\hline Negativo \\
\hline Negativo \\
\hline Positivo \\
\hline Negativo \\
\hline Negativo \\
\hline Negativo \\
\hline Negativo \\
\hline Negativo \\
\hline Negativo \\
\hline Negativo \\
\hline Negativo \\
\hline Positivo \\
\hline Negativo \\
\hline Negativo \\
\hline Negativo \\
\hline Negativo \\
\hline Negativo \\
\hline Negativo \\
\hline Negativo \\
\hline Negativo \\
\hline Negativo \\
\hline Negativo \\
\hline
\end{tabular}




\begin{tabular}{|c|}
\hline Negativo \\
\hline Negativo \\
\hline Negativo \\
\hline Negativo \\
\hline Negativo \\
\hline Negativo \\
\hline Negativo \\
\hline Negativo \\
\hline Negativo \\
\hline Negativo \\
\hline Negativo \\
\hline Negativo \\
\hline Negativo \\
\hline Positivo \\
\hline Negativo \\
\hline Negativo \\
\hline Negativo \\
\hline Negativo \\
\hline Negativo \\
\hline Negativo \\
\hline Negativo \\
\hline Negativo \\
\hline Negativo \\
\hline Negativo \\
\hline Negativo \\
\hline Negativo \\
\hline Negativo \\
\hline Negativo \\
\hline Negativo \\
\hline Negativo \\
\hline Negativo \\
\hline Negativo \\
\hline Negativo \\
\hline Negativo \\
\hline Negativo \\
\hline Negativo \\
\hline Negativo \\
\hline Negativo \\
\hline Negativo \\
\hline Negativo \\
\hline Negativo \\
\hline Negativo \\
\hline Negativo \\
\hline Negativo \\
\hline Negativo \\
\hline Negativo \\
\hline Negativo \\
\hline Negativo \\
\hline Negativo \\
\hline Negativo \\
\hline
\end{tabular}




\begin{tabular}{|l|}
\hline Negativo \\
\hline Negativo \\
\hline Negativo \\
\hline Negativo \\
\hline Negativo \\
\hline Negativo \\
\hline Negativo \\
\hline Negativo \\
\hline Negativo \\
\hline Negativo \\
\hline Negativo \\
\hline Negativo \\
\hline Negativo \\
\hline Positivo \\
\hline Negativo \\
\hline Negativo \\
\hline Negativo \\
\hline Negativo \\
\hline Negativo \\
\hline Negativo \\
\hline Negativo \\
\hline Negativo \\
\hline Negativo \\
\hline
\end{tabular}


Data of molecular biology

\begin{tabular}{|c|c|c|c|c|}
\hline Type of sample & Patient & Age & Sex & Result \\
\hline Hemocultura & B1 & 57 & $M$ & Negativo \\
\hline Sangue & B2 & 54 & $\mathrm{M}$ & Negativo \\
\hline Liquor/LCR & B3 & 35 & $\mathrm{~F}$ & Negativo \\
\hline Sangue & B4 & 35 & $\mathrm{~F}$ & Negativo \\
\hline Sangue & B5 & 67 & $\mathrm{~F}$ & Negativo \\
\hline Sangue & B6 & 74 & $\mathrm{M}$ & Negativo \\
\hline Hemocultura & B7 & 11 & $\mathrm{M}$ & Brucella melitensis \\
\hline Liquor/LCR & B8 & 42 & $\mathrm{~F}$ & Negativo \\
\hline Estirpe & B9 & 72 & $M$ & Brucella melitensis \\
\hline Liquor/LCR & B10 & 0 & $M$ & Negativo \\
\hline Sangue & B11 & 82 & $\mathrm{~F}$ & Negativo \\
\hline Sangue & B12 & 67 & $\mathrm{~F}$ & Negativo \\
\hline Liquor/LCR & B13 & 67 & $\mathrm{~F}$ & Negativo \\
\hline Sangue & B14 & 67 & $\mathrm{~F}$ & Negativo \\
\hline Liquor/LCR & B15 & 46 & $M$ & Negativo \\
\hline Sangue & B16 & 82 & $\mathrm{~F}$ & Negativo \\
\hline Liquor/LCR & B17 & 76 & $M$ & Negativo \\
\hline Liquor/LCR & B18 & 81 & $\mathrm{M}$ & Negativo \\
\hline Liquor/LCR & B19 & 62 & $M$ & Negativo \\
\hline Liquor/LCR & B20 & 61 & $\mathrm{M}$ & Negativo \\
\hline Sangue & B21 & 61 & $\mathrm{M}$ & Negativo \\
\hline Sangue & B22 & 71 & $\mathrm{~F}$ & Negativo \\
\hline Sangue & B23 & 71 & $\mathrm{~F}$ & Negativo \\
\hline Biopsia & B24 & 34 & $\mathrm{~F}$ & Negativo \\
\hline Sangue & B25 & 74 & M & Negativo \\
\hline Biopsia & B26 & 18 & $\mathrm{~F}$ & Negativo \\
\hline Liquor/LCR & $\mathrm{B} 27$ & 18 & $\mathrm{~F}$ & Negativo \\
\hline Sangue & B28 & 14 & M & Negativo \\
\hline Liquor/LCR & B29 & 41 & $M$ & Negativo \\
\hline Sangue & B30 & 17 & $\mathrm{~F}$ & Negativo \\
\hline Estirpe & B31 & 53 & $M$ & Brucella melitensis \\
\hline Liquor/LCR & B32 & 64 & $M$ & Negativo \\
\hline Liquor/LCR & B33 & 73 & $M$ & Negativo \\
\hline Estirpe & B34 & 0 & $\mathrm{~F}$ & Brucella melitensis \\
\hline Hemocultura & B35 & 46 & $M$ & Negativo \\
\hline Sangue & B36 & 20 & $\mathrm{~F}$ & Negativo \\
\hline Estirpe & B37 & 47 & $\mathrm{~F}$ & Brucella melitensis \\
\hline Biopsia & B38 & 68 & $\mathrm{~F}$ & Negativo \\
\hline Sangue & B39 & 68 & $\mathrm{~F}$ & Negativo \\
\hline Sangue & B40 & 74 & $M$ & Negativo \\
\hline Biopsia & B41 & 76 & $\mathrm{~F}$ & Negativo \\
\hline Liquor/LCR & B42 & 65 & $M$ & Negativo \\
\hline Hemocultura & B43 & 54 & M & Brucella melitensis \\
\hline Sangue & B44 & 48 & $\mathrm{~F}$ & Negativo \\
\hline Sangue & B45 & 76 & $\mathrm{~F}$ & Negativo \\
\hline Sangue & B46 & 72 & M & Negativo \\
\hline Hemocultura & B47 & 30 & $\mathrm{~F}$ & Negativo \\
\hline Sangue & B48 & 63 & $M$ & Negativo \\
\hline
\end{tabular}




\begin{tabular}{|c|c|c|c|c|}
\hline Sangue & B49 & 30 & $\mathrm{~F}$ & Negativo \\
\hline Sangue & B50 & 49 & $\mathrm{~F}$ & Negativo \\
\hline Sangue & B51 & 30 & $\mathrm{~F}$ & Negativo \\
\hline Sangue & B52 & 80 & M & Negativo \\
\hline Sangue & B53 & 52 & $M$ & Negativo \\
\hline Sangue & B54 & 41 & $M$ & Negativo \\
\hline Sangue & B55 & 30 & $\mathrm{~F}$ & Negativo \\
\hline Sangue & B56 & 65 & $\mathrm{~F}$ & Negativo \\
\hline Liquor/LCR & B57 & 70 & $\mathrm{~F}$ & Negativo \\
\hline Hemocultura & B58 & 84 & $\mathrm{~F}$ & Negativo \\
\hline Liquor/LCR & B59 & 53 & $M$ & Negativo \\
\hline Liquor/LCR & $\mathrm{B} 60$ & 56 & M & Negativo \\
\hline Sangue & B61 & 56 & $M$ & Negativo \\
\hline Liquor/LCR & B62 & 58 & $M$ & Negativo \\
\hline Estirpe & B63 & 87 & $M$ & Brucella melitensis \\
\hline Liquor/LCR & B64 & 0 & $\mathrm{~F}$ & Negativo \\
\hline Biopsia & B65 & 68 & $M$ & Brucella melitensis \\
\hline Sangue & B66 & 68 & $M$ & Negativo \\
\hline Sangue & $\mathrm{B} 67$ & 61 & $\mathrm{~F}$ & Negativo \\
\hline Liquor/LCR & B68 & 88 & $\mathrm{~F}$ & Negativo \\
\hline Liquor/LCR & B69 & 61 & $M$ & Negativo \\
\hline Liquor/LCR & B70 & 82 & $\mathrm{~F}$ & Brucella melitensis \\
\hline Sangue & B71 & 53 & $\mathrm{~F}$ & Negativo \\
\hline Sangue & B72 & 76 & $\mathrm{~F}$ & Negativo \\
\hline Sangue & B73 & 40 & $\mathrm{~F}$ & Negativo \\
\hline Liquor/LCR & B74 & 83 & $M$ & Negativo \\
\hline Sangue & B75 & 74 & $M$ & Negativo \\
\hline Sangue & B76 & 41 & $M$ & Negativo \\
\hline Sangue & B77 & 54 & $\mathrm{~F}$ & Negativo \\
\hline Sangue & B78 & 52 & $M$ & Negativo \\
\hline Sangue & B79 & 63 & $M$ & Brucella melitensis \\
\hline Sangue & B80 & 40 & $M$ & Negativo \\
\hline Liquor/LCR & B81 & 66 & $M$ & Negativo \\
\hline Sangue & B82 & 73 & $M$ & Negativo \\
\hline Liquor/LCR & B83 & 65 & $\mathrm{~F}$ & Negativo \\
\hline Liquor/LCR & B84 & 56 & $M$ & Negativo \\
\hline Estirpe & B85 & 61 & $M$ & Negativo \\
\hline Liquor/LCR & B86 & 70 & $M$ & Negativo \\
\hline Sangue & B87 & 36 & $M$ & Negativo \\
\hline Liquor/LCR & B88 & 36 & $M$ & Negativo \\
\hline Liquor/LCR & B89 & 31 & $\mathrm{~F}$ & Negativo \\
\hline Biopsia & B90 & 54 & $M$ & Negativo \\
\hline Sangue & B91 & 46 & $M$ & Negativo \\
\hline Liquor/LCR & B92 & 49 & $\mathrm{~F}$ & Negativo \\
\hline Estirpe & B93 & 50 & $\mathrm{~F}$ & Brucella melitensis \\
\hline Sangue & B94 & 41 & $\mathrm{~F}$ & Negativo \\
\hline Sangue & B95 & 8 & $\mathrm{~F}$ & Negativo \\
\hline Sangue & B96 & 4 & $\mathrm{~F}$ & Negativo \\
\hline Liquor/LCR & B97 & 81 & $M$ & Negativo \\
\hline Biopsia & B98 & 64 & $M$ & Negativo \\
\hline
\end{tabular}




\begin{tabular}{|c|c|c|c|c|}
\hline Sangue & B99 & 18 & $M$ & Negativo \\
\hline Biopsia & B100 & 59 & 1 & Negativo \\
\hline Biopsia & B101 & 59 & 1 & Negativo \\
\hline Sangue & B102 & 5 & $\mathrm{~F}$ & Negativo \\
\hline Sangue & B103 & 5 & $\mathrm{~F}$ & Negativo \\
\hline Liquor/LCR & B104 & 50 & $M$ & Negativo \\
\hline Biopsia & B105 & 19 & $M$ & Negativo \\
\hline Sangue & B106 & 19 & $\mathrm{~F}$ & Negativo \\
\hline Liquor/LCR & B107 & 45 & $M$ & Negativo \\
\hline Liquor/LCR & B108 & 51 & $\mathrm{~F}$ & Negativo \\
\hline Sangue & B109 & 38 & $\mathrm{~F}$ & Negativo \\
\hline Liquor/LCR & B110 & 77 & $M$ & Negativo \\
\hline Liquor/LCR & B111 & 52 & $M$ & Negativo \\
\hline Liquor/LCR & B112 & 89 & $\mathrm{~F}$ & Negativo \\
\hline Liquor/LCR & B113 & 67 & $M$ & Negativo \\
\hline Sangue & B114 & 31 & $M$ & Negativo \\
\hline Liquor/LCR & B115 & 37 & $F$ & Negativo \\
\hline Liquor/LCR & B116 & 55 & $M$ & Negativo \\
\hline Liquor/LCR & B117 & 48 & $\mathrm{~F}$ & Negativo \\
\hline Exsudado & B118 & 0 & $M$ & Brucella melitensis \\
\hline Sangue & B119 & 0 & $M$ & Brucella melitensis \\
\hline Exsudado & B120 & 0 & $M$ & Brucella melitensis \\
\hline Sangue & B121 & 0 & $M$ & Brucella melitensis \\
\hline Exsudado & B122 & 0 & $M$ & Brucella melitensis \\
\hline Sangue & B123 & 0 & $M$ & Brucella melitensis \\
\hline Sangue & B124 & 0 & $M$ & Brucella melitensis \\
\hline Exsudado & B125 & 0 & $M$ & Brucella melitensis \\
\hline Exsudado & B126 & 0 & $M$ & Brucella melitensis \\
\hline Sangue & B127 & 0 & $M$ & Brucella melitensis \\
\hline Sangue & B128 & 0 & $M$ & Brucella melitensis \\
\hline Exsudado & B129 & 0 & $M$ & Brucella melitensis \\
\hline Sangue & B130 & 0 & $M$ & Brucella melitensis \\
\hline Exsudado & B131 & 0 & $M$ & Brucella melitensis \\
\hline Sangue & B132 & 0 & $M$ & Brucella melitensis \\
\hline Exsudado & B133 & 0 & $M$ & Brucella melitensis \\
\hline Sangue & B134 & 17 & $\mathrm{~F}$ & Negativo \\
\hline Liquor/LCR & B135 & 79 & $\mathrm{~F}$ & Negativo \\
\hline Liquor/LCR & B136 & 52 & $M$ & Negativo \\
\hline Liquor/LCR & B137 & 33 & $M$ & Negativo \\
\hline Liquor/LCR & B138 & 29 & $M$ & Negativo \\
\hline Liquor/LCR & B139 & 55 & $\mathrm{~F}$ & Negativo \\
\hline Liquor/LCR & B140 & 82 & $\mathrm{~F}$ & Negativo \\
\hline Sangue & B141 & 19 & $M$ & Negativo \\
\hline Liquor/LCR & B142 & 33 & $\mathrm{~F}$ & Negativo \\
\hline Liquor/LCR & B143 & 83 & $M$ & Negativo \\
\hline Liquor/LCR & B144 & 40 & $M$ & Negativo \\
\hline Liquor/LCR & B145 & 55 & $\mathrm{~F}$ & Negativo \\
\hline Liquor/LCR & B146 & 46 & $M$ & Negativo \\
\hline Sangue & B147 & 43 & $\mathrm{~F}$ & Negativo \\
\hline Liquor/LCR & B148 & 43 & $\mathrm{~F}$ & Negativo \\
\hline
\end{tabular}




\begin{tabular}{|c|c|c|c|c|}
\hline Liquor/LCR & B149 & 75 & $M$ & Negativo \\
\hline Liquor/LCR & B150 & 71 & $\mathrm{~F}$ & Negativo \\
\hline Liquor/LCR & B151 & 78 & $\mathrm{~F}$ & Negativo \\
\hline Liquor/LCR & B152 & 32 & $M$ & Negativo \\
\hline Liquor/LCR & B153 & 85 & $\mathrm{~F}$ & Negativo \\
\hline Liquor/LCR & B154 & 61 & $\mathrm{~F}$ & Negativo \\
\hline Liquor/LCR & B155 & 71 & $\mathrm{~F}$ & Negativo \\
\hline Liquor/LCR & B156 & 27 & $\mathrm{~F}$ & Negativo \\
\hline Liquor/LCR & B157 & 68 & $M$ & Negativo \\
\hline Liquor/LCR & B158 & 32 & $M$ & Negativo \\
\hline Liquor/LCR & B159 & 62 & $M$ & Negativo \\
\hline Sangue & B160 & 74 & $M$ & Negativo \\
\hline Liquor/LCR & B161 & 48 & $M$ & Negativo \\
\hline Liquor/LCR & B162 & 66 & $M$ & Negativo \\
\hline Liquor/LCR & B163 & 91 & $M$ & Negativo \\
\hline Liquor/LCR & B164 & 25 & $M$ & Negativo \\
\hline Sangue & B165 & 25 & $M$ & Negativo \\
\hline Liquor/LCR & B166 & 66 & $M$ & Negativo \\
\hline Liquor/LCR & B167 & 80 & $M$ & Negativo \\
\hline Liquor/LCR & B168 & 35 & $\mathrm{~F}$ & Negativo \\
\hline Liquor/LCR & B169 & 71 & $\mathrm{~F}$ & negativo \\
\hline Liquor/LCR & B170 & 75 & $M$ & Negativo \\
\hline Liquor/LCR & B171 & 77 & $M$ & Negativo \\
\hline Liquor/LCR & B172 & 81 & $M$ & Negativo \\
\hline Liquor/LCR & B173 & 64 & $\mathrm{~F}$ & Negativo \\
\hline Liquor/LCR & B174 & 68 & $M$ & Negativo \\
\hline Biopsia & B175 & 60 & $M$ & Negativo \\
\hline Liquor/LCR & B176 & 39 & $M$ & Negativo \\
\hline Liquor/LCR & B177 & 75 & $M$ & Negativo \\
\hline Liquor/LCR & B178 & 30 & $M$ & Negativo \\
\hline Sangue & B179 & 30 & $M$ & Negativo \\
\hline Liquor/LCR & B180 & 51 & $\mathrm{~F}$ & Negativo \\
\hline Liquor/LCR & B181 & 51 & $\mathrm{~F}$ & Negativo \\
\hline Liquor/LCR & B182 & 69 & $M$ & Negativo \\
\hline Liquor/LCR & B183 & 81 & $\mathrm{~F}$ & Negativo \\
\hline Liquor/LCR & B184 & 45 & $M$ & Negativo \\
\hline Liquor/LCR & B185 & 62 & $\mathrm{~F}$ & Negativo \\
\hline Sangue & B186 & 16 & $\mathrm{~F}$ & Negativo \\
\hline Liquor/LCR & B187 & 81 & $M$ & Negativo \\
\hline Liquor/LCR & B188 & 66 & $M$ & Negativo \\
\hline Liquor/LCR & B189 & 26 & $M$ & Negativo \\
\hline Liquor/LCR & B190 & 60 & $M$ & Negativo \\
\hline Liquor/LCR & B191 & 50 & $\mathrm{~F}$ & Negativo \\
\hline Liquor/LCR & B192 & 61 & $M$ & Negativo \\
\hline Liquor/LCR & B193 & 67 & $\mathrm{~F}$ & Negativo \\
\hline Liquor/LCR & B194 & 75 & $\mathrm{~F}$ & Negativo \\
\hline Liquor/LCR & B195 & 88 & $M$ & Negativo \\
\hline Liquor/LCR & B196 & 82 & $\mathrm{~F}$ & Negativo \\
\hline Sangue & B197 & 66 & M & Negativo \\
\hline Liquor/LCR & B198 & 66 & $M$ & Negativo \\
\hline
\end{tabular}




\begin{tabular}{|c|c|c|c|c|}
\hline Liquor/LCR & B199 & 37 & $M$ & Negativo \\
\hline Biopsia & B200 & 51 & $M$ & Brucella melitensis \\
\hline Liquor/LCR & B201 & 54 & $\mathrm{~F}$ & Negativo \\
\hline Estirpe & B202 & 57 & $\mathrm{~F}$ & Negativo \\
\hline Liquor/LCR & B203 & 33 & $F$ & Negativo \\
\hline Liquor/LCR & B204 & 86 & $M$ & Negativo \\
\hline Liquor/LCR & B205 & 84 & $\mathrm{~F}$ & Negativo \\
\hline Liquor/LCR & B206 & 80 & $M$ & Negativo \\
\hline Liquor/LCR & B207 & 45 & $M$ & Negativo \\
\hline Liquor/LCR & B208 & 79 & $M$ & Negativo \\
\hline Liquor/LCR & B209 & 73 & $\mathrm{~F}$ & Negativo \\
\hline Sangue & B210 & 50 & I & Negativo \\
\hline Sangue & B211 & 82 & I & Negativo \\
\hline Biopsia & B212 & 83 & 1 & Brucella melitensis \\
\hline Sangue & B213 & 77 & I & Negativo \\
\hline Estirpe & B214 & 67 & 1 & Brucella melitensis \\
\hline Estirpe & B215 & 9 & 1 & Brucella melitensis \\
\hline Estirpe & B216 & 41 & I & brucella melitensis \\
\hline Estirpe & B217 & 43 & 1 & brucella melitensis \\
\hline Estirpe & B218 & 64 & I & Brucella melitensis \\
\hline Estirpe & B219 & 55 & 1 & Brucella melitensis \\
\hline Estirpe & B220 & 52 & $M$ & Brucella melitensis \\
\hline Liquor/LCR & B221 & 75 & $\mathrm{M}$ & Negativo \\
\hline Estirpe & B222 & 72 & $F$ & Brucella melitensis \\
\hline Estirpe & B223 & 14 & $\mathrm{M}$ & Brucella melitensis \\
\hline Liquor/LCR & B224 & 55 & $M$ & Negativo \\
\hline Estirpe & B225 & 46 & $F$ & Brucella melitensis \\
\hline Sangue & B226 & 15 & $\mathrm{M}$ & Negativo \\
\hline Sangue & B227 & 23 & $F$ & Negativo \\
\hline Liquor/LCR & B228 & 23 & $F$ & Negativo \\
\hline Liquor/LCR & B229 & 69 & $\mathrm{M}$ & Negativo \\
\hline Liquido sinovial & B230 & 46 & $\mathrm{M}$ & Negativo \\
\hline Sangue & B231 & 17 & $\mathrm{M}$ & Negativo \\
\hline Liquor/LCR & B232 & 17 & $\mathrm{M}$ & Negativo \\
\hline Liquor/LCR & B233 & 61 & $M$ & Negativo \\
\hline Liquor/LCR & B234 & 86 & $M$ & Negativo \\
\hline Liquor/LCR & B235 & 56 & $M$ & Negativo \\
\hline Sangue & B236 & 90 & $F$ & Negativo \\
\hline Biopsia & B237 & 59 & $F$ & Negativo \\
\hline Sangue & B238 & 20 & $\mathrm{M}$ & Negativo \\
\hline Sangue & B239 & 59 & $\mathrm{~F}$ & Negativo \\
\hline Sangue & B240 & 49 & $M$ & Negativo \\
\hline Liquor/LCR & B241 & 27 & $F$ & Negativo \\
\hline Sangue & B242 & 21 & $\mathrm{~F}$ & Negativo \\
\hline Sangue & B243 & 78 & $F$ & Negativo \\
\hline Liquor/LCR & B244 & 11 & $\mathrm{M}$ & Negativo \\
\hline Estirpe & B245 & 64 & $M$ & Brucella mellitensis \\
\hline Biopsia & B246 & 38 & $M$ & Brucella melitensis \\
\hline Estirpe & B247 & 65 & $\mathrm{M}$ & Brucella mellitensis \\
\hline Sangue & B248 & 0 & $\mathrm{~F}$ & Negativo \\
\hline
\end{tabular}




$\begin{array}{lllll}\text { Estirpe } & \text { B249 } & 60 & \mathrm{~F} & \\ \text { Liquor/LCR } & \text { B250 } & 56 & \text { M } & \text { Negativo } \\ \text { Liquor/LCR } & \text { B251 } & 50 & \text { M } & \text { Negativo } \\ \text { Liquor/LCR } & \text { B252 } & 63 & \mathrm{~F} & \text { Negativo } \\ \text { Liquor/LCR } & \text { B253 } & 71 & \text { M } & \text { Negativo } \\ \text { Sangue } & \text { B254 } & 43 & \mathrm{~F} & \text { Negativo } \\ \text { Liquor/LCR } & \text { B255 } & 73 & \text { M } & \text { Negativo } \\ \text { Liquor/LCR } & \text { B256 } & 69 & \text { M } & \text { Negativo } \\ \text { Biopsia } & \text { B257 } & 50 & \text { M } & \text { Negativo } \\ \text { Medula óssea } & \text { B258 } & 50 & \text { M } & \text { Negativo } \\ \text { Sangue } & \text { B259 } & 9 & \text { M } & \text { Negativo }\end{array}$

